\title{
AN INTRODUCTION TO SHELL THEORY
}

\author{
PHILIPPE G. CIARLET AND CRISTINEL MARDARE
}

Abstract. These notes are intended to provide a thorough introduction to the mathematical theory of elastic shells.

The main objective of shell theory is to predict the stress and the displacement arising in an elastic shell in response to given forces. Such a prediction is made either by solving a system of partial differential equations or by minimizing a functional, which may be defined either over a three-dimensional set or over a two-dimensional set, depending on whether the shell is viewed in its reference configuration as a three-dimensional or as a two-dimensional body (the latter being an abstract idealization of the physical shell when its thickness is "small").

The first part of this article is devoted to the three-dimensional theory of elastic bodies, from which the three-dimensional theory of shells is obtained simply by replacing the reference configuration of a general body with that of a shell. The particular shape of the reference configuration of the shell does not play any rôle in this theory.

The second part is devoted to the two-dimensional theory of elastic shells. In contrast to the three-dimensional theory, this theory is specific to shells, since it essentially depends on the geometry of the reference configuration of a shell.

For a more comprehensive exposition of the theory of elastic shells, we refer the reader to Ciarlet [18] and the references therein for the first part of the article, and to Ciarlet $[20]$ and the references therein for the second part.

\section{Une introduction a la théorie des coques}

Resumé. Ces notes sont destinées à fournir une introduction détaillée à la théorie mathématique des coques élastiques.

L'objectif principal de la théorie des coques est de prédire les contraintes et les déplacements survenant dans une coque élastique en réponse à des forces appliquées. Une telle prédiction est faite soit en résolvant un système d'équations aux dérivées partielles, soit en minimisant une fonctionnelle, qui peuvent être définis soit dans un ensemble tridimensionnel, soit dans un ensemble bidimensionnel, selon que la coque est vue dans sa configuration de référence comme un corps tridimensionnel ou comme un corps bidimensionnel (ce dernier étant alors une idéalisation de la coque physique lorsque son épaisseur est "petite").

La première partie de cet article est consacrée à la théorie tridimensionnelle des corps élastiques, à partir de laquelle la théorie tridimensionnelle des coques est obtenue en remplaçant simplement la configuration de référence d'un corps élastique général par celle d'une coque. La forme particulière de la configuration de référence d'une coque ne joue aucun rôle dans cette théorie.

La deuxième partie de cet article est consacrée à la théorie bidimensionnelle des coques élastiques. Contrairement à la théorie tridimensionnelle, cette théorie est spécifique aux coques, puisqu'elle dépend de façon essentielle de la géométrie de la configuration de référence de la coque.

Pour un exposé plus complet de la théorie de coques élastiques, nous renvoyons le lecteur à Ciarlet [18] et ses références pour la première partie de cet article, et à Ciarlet [20] et ses références pour la deuxième. 


\section{Contents}

Part 1. Three-Dimensional theory 1 Outline

1.1. Notation, definitions, and some basic formulas 1

1.2. Equations of equilibrium 3

1.3. Constitutive equations of elastic materials 6

1.4. The equations of nonlinear and linearized three-dimensional elasticity 9

1.5. A fundamental lemma of J.L. Lions 11

1.6. Existence theory in linearized three-dimensional elasticity 12

1.7. Existence theory in nonlinear three-dimensional elasticity by the implicit function theorem

1.8. Existence theory in nonlinear three-dimensional elasticity by the minimization of energy (John Ball's approach)

PART 2. Two-Dimensional THEORY 22

Outline 22

2.1. A quick review of the differential geometry of surfaces in $\mathbb{R}^{3} \quad 22$

2.2. Geometry of a shell 24

2.3. The three-dimensional shell equations 27

2.4. The two-dimensional approach to shell theory 29

2.5. Nonlinear shell models obtained by $\Gamma$-convergence 30

2.6. Linear shell models obtained by asymptotic analysis 37

2.7. The nonlinear Koiter shell model $\quad 42$

2.8. The linear Koiter shell model 44

2.9. Korn's inequalities on a surface 50

2.10. Existence, uniqueness, and regularity of the solution to the linear Koiter shell model $\quad 60$

References 


\section{Part 1. THREE-DIMENSIONAL THEORY}

\section{OUTLINE}

In this first part of the article, the displacement and the stress arising in an elastic shell, or for that matter in any three-dimensional elastic body, in response to given loads are predicted by means of a system of partial differential equations in three variables (the coordinates of the physical space). This system is formed either by the equations of nonlinear three-dimensional elasticity or by the equations of linearized three-dimensional elasticity.

Sections 1.2-1.4 are devoted to the derivation of the equations of three-dimensional elasticity in the form of two basic sets of equations, the equations of equilibrium and the constitutive equations. The equations of nonlinear three-dimensional elasticity are then obtained by adjoining appropriate boundary conditions to these equations. The equations of linearized three-dimensional elasticity are obtained from the nonlinear ones by linearization with respect to the displacement field.

Sections 1.5-1.6 study the existence and uniqueness of solutions to the equations of linearized three-dimensional elasticity.

Using a fundamental lemma, due to J.L. Lions, about distributions with derivatives in "negative" Sobolev spaces (Section 1.5), we establish in Section 1.6 the fundamental Korn inequality, which in turn implies that the equations of linearized three-dimensional elasticity have a unique solution.

In sections 1.7-1.8, we study the existence of solutions to the equations of nonlinear three-dimensional elasticity, which fall into two distinct categories:

If the data are regular, the applied forces are "small", and the boundary condition does not change its nature along connected portions of the boundary, the equations of nonlinear three-dimensional elasticity have a solution by the implicit function theorem (Section 1.7).

If the constituting material is hyperelastic and the associated stored energy function satisfies certain conditions of polyconvexity, coerciveness, and growth, the minimization problem associated with the equations of nonlinear three-dimensional elasticity has a solution by a fundamental theorem of John Ball (Section 1.8).

\subsection{Notation, DEFINITIONS, AND SOME BASIC FORMUlAS}

All spaces, matrices, etc., are real. The Kronecker symbol is denoted $\delta_{i}^{j}$.

The physical space is identified with the three-dimensional vector space $\mathbb{R}^{3}$ by fixing an origin and a cartesian basis $\left(\mathbf{e}_{1}, \mathbf{e}_{2}, \mathbf{e}_{3}\right)$. In this way, a point $x$ in space is defined by its cartesian coordinates $x_{1}, x_{2}, x_{3}$ or by the vector $\boldsymbol{x}:=\sum_{i} x_{i} \mathbf{e}_{i}$. The space $\mathbb{R}^{3}$ is equipped with the Euclidean inner product $\boldsymbol{u} \cdot \boldsymbol{v}$ and with the Euclidean norm $|\boldsymbol{u}|$, where $\boldsymbol{u}, \boldsymbol{v}$ denote vectors in $\mathbb{R}^{3}$. The exterior product of two vectors $\boldsymbol{u}, \boldsymbol{v} \in \mathbb{R}^{3}$ is denoted $\boldsymbol{u} \wedge \boldsymbol{v}$.

For any integer $n \geq 2$, we define the following spaces or sets of real square matrices of order $n$ :

$\mathbb{M}^{n}$ : the space of all square matrices,

$\mathbb{A}^{n}$ : the space of all anti-symmetric matrices,

$\mathbb{S}^{n}$ : the space of all symmetric matrices,

$\mathbb{M}_{+}^{n}$ : the set of all matrices $\boldsymbol{A} \in \mathbb{M}^{n}$ with $\operatorname{det} \boldsymbol{A}>0$,

$\mathbb{S}_{>}^{n}$ : the set of all positive-definite symmetric matrices,

$\mathbb{O}^{n}$ : the set of all orthogonal matrices, 
$\mathbb{O}_{+}^{n}$ : the set of all orthogonal matrices $\boldsymbol{R} \in \mathbb{O}^{n}$ with $\operatorname{det} \boldsymbol{R}=1$.

The notation $\left(a_{i j}\right)$ designates the matrix in $\mathbb{M}^{n}$ with $a_{i j}$ as its element at the $i$-th row and $j$-th column. The identity matrix in $\mathbb{M}^{n}$ is denoted $\boldsymbol{I}:=\left(\delta_{j}^{i}\right)$. The space $\mathbb{M}^{n}$, and its subspaces $\mathbb{A}^{n}$ and $\mathbb{S}^{n}$ are equipped with the inner product $\boldsymbol{A}: \boldsymbol{B}$ and with the spectral norm $|\boldsymbol{A}|$ defined by

$$
\begin{aligned}
& \boldsymbol{A}: \boldsymbol{B}:=\sum_{i, j} a_{i j} b_{i j}, \\
& |\boldsymbol{A}|:=\sup \left\{|\boldsymbol{A} \boldsymbol{v}| ; \boldsymbol{v} \in \mathbb{R}^{n},|\boldsymbol{v}| \leq 1\right\},
\end{aligned}
$$

where $\boldsymbol{A}=\left(a_{i j}\right)$ and $\boldsymbol{B}=\left(b_{i j}\right)$ denote matrices in $\mathbb{M}^{n}$. The determinant and the trace of a matrix $\boldsymbol{A}=\left(a_{i j}\right)$ are $\operatorname{denoted} \operatorname{det} \boldsymbol{A}$ and $\operatorname{tr} \boldsymbol{A}$. The cofactor matrix associated with an invertible matrix $\boldsymbol{A} \in \mathbb{M}^{n}$ is defined by $\operatorname{Cof} \boldsymbol{A}:=(\operatorname{det} \boldsymbol{A}) \boldsymbol{A}^{-T}$.

Let $\Omega$ be an open subset of $\mathbb{R}^{3}$. Partial derivative operators of order $m \geq 1$ acting on functions or distributions defined over $\Omega$ are denoted

$$
\partial^{\boldsymbol{k}}:=\frac{\partial^{|\boldsymbol{k}|}}{\partial x_{1}^{k_{1}} \partial x_{2}^{k_{2}} \partial x_{3}^{k_{3}}}
$$

where $\boldsymbol{k}=\left(k_{i}\right) \in \mathbb{N}^{3}$ is a multi-index satisfying $|\boldsymbol{k}|:=k_{1}+k_{2}+k_{3}=m$. Partial derivative operators of the first, second, and third order are also denoted $\partial_{i}:=$ $\partial / \partial x_{i}, \partial_{i j}:=\partial^{2} / \partial x_{i} \partial x_{j}$, and $\partial_{i j k}:=\partial^{3} / \partial x_{1} \partial x_{2} \partial x_{3}$.

The gradient of a function $f: \Omega \rightarrow \mathbb{R}$ is the vector field $\operatorname{grad} f:=\left(\partial_{i} f\right)$, where $i$ is the row index. The gradient of a vector field $\boldsymbol{v}=\left(v_{i}\right): \Omega \rightarrow \mathbb{R}^{n}$ is the matrix field $\boldsymbol{\nabla} \boldsymbol{v}:=\left(\partial_{j} v_{i}\right)$, where $i$ is the row index, and the divergence of the same vector field is the function $\operatorname{div} \boldsymbol{v}:=\sum_{i} \partial_{i} v_{i}$. Finally, the divergence of a matrix field $\boldsymbol{T}=\left(t_{i j}\right): \Omega \rightarrow \mathbb{M}^{n}$ is the vector field $\operatorname{div} \boldsymbol{T}$ with components $\left(\sum_{j=1}^{n} \partial_{j} t_{i j}\right)_{i}$.

The space of all continuous functions from a topological space $X$ into a normed space $Y$ is denoted $\mathcal{C}^{0}(X ; Y)$, or simply $\mathcal{C}^{0}(X)$ if $Y=\mathbb{R}$.

For any integer $m \geq 1$ and any open set $\Omega \subset \mathbb{R}^{3}$, the space of all real-valued functions that are $m$ times continuously differentiable over $\Omega$ is denoted $\mathcal{C}^{m}(\Omega)$. The space $\mathcal{C}^{m}(\bar{\Omega}), m \geq 1$, is defined as that consisting of all vector-valued functions $f \in \mathcal{C}^{m}(\Omega)$ that, together with all their partial derivatives of order $\leq m$, possess continuous extentions to the closure $\bar{\Omega}$ of $\Omega$. If $\Omega$ is bounded, the space $\mathcal{C}^{m}(\bar{\Omega})$ equipped with the norm

$$
\|f\|_{\mathcal{C}^{m}(\bar{\Omega})}:=\max _{|\boldsymbol{\alpha}| \leq m}\left(\sup _{x \in \Omega}\left|\partial^{\boldsymbol{\alpha}} f(x)\right|\right)
$$

is a Banach space.

The space of all indefinitely derivable functions $\varphi: \Omega \rightarrow \mathbb{R}$ with compact support contained in $\Omega$ is denoted $\mathcal{D}(\Omega)$ and the space of all distributions over $\Omega$ is denoted $\mathcal{D}^{\prime}(\Omega)$. The duality bracket between a distribution $T$ and a test function $\varphi \in \mathcal{D}(\Omega)$ is denoted $\langle T, \varphi\rangle$.

The usual Lebesgue and Sobolev spaces are respectively denoted $L^{p}(\Omega)$, and $W^{m, p}(\Omega)$ for any integer $m \geq 1$ and any $p \geq 1$. If $p=2$, we use the notation $H^{m}(\Omega)=W^{m, 2}(\Omega)$. The space $W_{\mathrm{loc}}^{m, p}(\Omega)$ is the space of all mesurable functions such that $\left.f\right|_{U} \in W^{m, p}(U)$ for all $U \Subset \Omega$, where the notation $\left.f\right|_{U}$ designates the restriction to the set $U$ of a function $f$ and the notation $U \Subset \Omega$ means that $\bar{U}$ is a compact set that satisfies $\bar{U} \subset \Omega$. 
The space $W_{0}^{m, p}(\Omega)$ is the closure of $\mathcal{D}(\Omega)$ in $W^{m, p}(\Omega)$ and the dual of the space $W_{0}^{m, p}(\Omega)$ is denoted $W^{-m, p^{\prime}}(\Omega)$, where $p^{\prime}=\frac{p}{p-1}$. If the boundary of $\Omega$ is Lipschitzcontinuous and if $\Gamma_{0} \subset \partial \Omega$ is a relatively open subset of the boundary of $\Omega$, we let

$$
\begin{aligned}
& W_{\Gamma_{0}}^{1, p}(\Omega):=\left\{f \in W^{1, p}(\Omega) ; f=0 \text { on } \Gamma_{0}\right\}, \\
& W_{\Gamma_{0}}^{2, p}(\Omega):=\left\{f \in W^{2, p}(\Omega) ; f=\partial_{\nu} f=0 \text { on } \Gamma_{0}\right\},
\end{aligned}
$$

where $\partial_{\nu}$ denote the outer normal derivative operator along $\partial \Omega$ (since $\Omega$ is Lipschitzcontinuous, a unit outer normal vector $\left(\nu_{i}\right)$ exists $\partial \Omega$-almost everywhere along $\partial \Omega$, and thus $\partial_{\nu}=\nu_{i} \partial_{i}$ ).

If $Y$ is a finite dimensional vectorial space (such as $\mathbb{R}^{n}, \mathbb{M}^{n}$, etc.), the notation $\mathcal{C}^{m}(\Omega ; Y), \mathcal{C}^{m}(\bar{\Omega} ; Y), L^{p}(\Omega ; Y)$ and $W^{m, p}(\Omega ; Y)$ designates the spaces of all mappings from $\Omega$ into $Y$ whose components in $Y$ are respectively in $\mathcal{C}^{m}(\Omega), \mathcal{C}^{m}(\bar{\Omega})$, $L^{p}(\Omega)$ and $W^{m, p}(\Omega)$. If $Y$ is equipped with the norm $|\cdot|$, then the spaces $L^{p}(\Omega ; Y)$ and $W^{m, p}(\Omega ; Y)$ are respectively equipped with the norms

$$
\|f\|_{L^{p}(\Omega ; Y)}:=\left\{\int_{\Omega}|f(x)|^{p} d x\right\}^{1 / p}
$$

and

$$
\|f\|_{W^{m, p}(\Omega ; Y)}:=\left\{\int_{\Omega}\left(|f(x)|^{p}+\sum_{|\boldsymbol{k}| \leq m}\left|\partial^{k} f(x)\right|^{p}\right) d x\right\}^{1 / p}
$$

Throughout this article, a domain in $\mathbb{R}^{n}$ is a bounded and connected open set with a Lipschitz-continuous boundary, the set $\Omega$ being locally on the same side of its boundary. See, e.g., Adams [2], Grisvard [54], or Nečas [73]. If $\Omega \subset \mathbb{R}^{n}$ is a domain, then the following formula of integration by parts is satisfied

$$
\int_{\Omega} \operatorname{div} \boldsymbol{F} \cdot \boldsymbol{v} d x=-\int_{\Omega} \boldsymbol{F}: \boldsymbol{\nabla} \boldsymbol{v} d x+\int_{\partial \Omega}(\boldsymbol{F} \boldsymbol{n}) \cdot \boldsymbol{v} d a
$$

for all smooth enough matrix field $\boldsymbol{F}: \Omega \rightarrow \mathbb{M}^{k}$ and vector field $\boldsymbol{v}: \Omega \rightarrow \mathbb{R}^{k}$, $k \geq 1$ (smooth enough means that the regularity of the fields $\boldsymbol{F}$ and $\boldsymbol{v}$ is such that the above integrals are well defined; for such instances, see, e.g., Evans \& Gariepy [47]). The notation $d a$ designates the area element induced on the surface $\partial \Omega$ by the volume element $d x$. We also record the Stokes formula:

$$
\int_{\Omega} \operatorname{div} \boldsymbol{F} d x=\int_{\partial \Omega} \boldsymbol{F} \boldsymbol{n} d a .
$$

\subsection{EquATIONS OF EQUILIBRIUM}

In this section, we begin our study of the deformation arising in an elastic body in response to given forces. We consider that the body occupies the closure of a domain $\Omega \subset \mathbb{R}^{3}$ in the absence of applied forces, henceforth called the reference configuration of the body. Any other configuration that the body might occupy when subjected to applied forces will be defined by means of a deformation, that is, a mapping

$$
\Phi: \bar{\Omega} \rightarrow \mathbb{R}^{3}
$$

that is orientation preserving (i.e., $\operatorname{det} \boldsymbol{\nabla} \Phi(x)>0$ for all $x \in \bar{\Omega}$ ) and injective on the open set $\Omega$ (i.e., no interpenetration of matter occurs). The image $\boldsymbol{\Phi}(\bar{\Omega})$ is called the deformed configuration of the body defined by the deformation $\boldsymbol{\Phi}$. The 
"difference" between a deformed configuration and the reference configuration is given by the displacement, which is the vector field defined by

$$
u:=\Phi-\mathrm{id}
$$

where id : $\bar{\Omega} \rightarrow \bar{\Omega}$ is the identity map. It is sometimes more convenient to describe the deformed configuration of a body in terms of the displacement $\boldsymbol{u}$ instead of the deformation $\boldsymbol{\Phi}$, notably when the body is expected to undergo small deformations (as typically occurs in linearized elasticity).

Our objective in this section is to determine, among all possible deformed configurations of the body, the ones that are in "static equilibrium" in the presence of applied forces. More specifically, let the applied forces acting on a specific deformed configuration $\tilde{\Omega}:=\boldsymbol{\Phi}(\Omega)$ be represented by the densities

$$
\tilde{\boldsymbol{f}}: \tilde{\Omega} \rightarrow \mathbb{R}^{3} \text { and } \tilde{\boldsymbol{g}}: \tilde{\Gamma}_{1} \rightarrow \mathbb{R}^{3},
$$

where $\tilde{\Gamma}_{1} \subset \partial \tilde{\Omega}$ is a relatively open subset of the boundary of $\tilde{\Omega}$.

If the body is subjected for instance to the gravity and to a uniform pressure on $\tilde{\Gamma}_{1}$, then the densities $\tilde{\boldsymbol{f}}$ and $\tilde{\boldsymbol{g}}$ are given by $\tilde{\boldsymbol{f}}(\tilde{x})=-g \tilde{\rho}(\tilde{x}) \mathbf{e}_{3}$ and $\tilde{\boldsymbol{g}}(\tilde{x})=-\pi \tilde{\boldsymbol{n}}(\tilde{x})$, where $g$ is the gravitational constant, $\tilde{\rho}: \tilde{\Omega} \rightarrow \mathbb{R}$ is the mass density in the deformed configuration, $\tilde{x}$ denotes a generic point in $\{\tilde{\Omega}\}^{-}, \tilde{\boldsymbol{n}}(\tilde{x})$ is the unit outer normal to $\partial \tilde{\Omega}$, and $\pi$ is a constant, called pressure.

These examples illustrate that an applied force density may, or may not, depend on the unknown deformation.

Our aim is thus to determine equations that a deformation $\mathbf{\Phi}$ corresponding to the static equilibrium of the loaded body should satisfy. To this end, we first derive the "equations of equilibrium" from a fundamental axiom due to Euler and Cauchy. The three-dimensional equations of elasticity will then be obtained by combining these equations with a "constitutive equation" (Section 1.3).

Let

$$
S_{2}:=\left\{\boldsymbol{v} \in \mathbb{R}^{3} ;|\boldsymbol{v}|=1\right\}
$$

denote the set of all unit vectors in $\mathbb{R}^{3}$. Then, according to the stress principle of Euler and Cauchy, a body $\tilde{\Omega} \subset \mathbb{R}^{3}$ subjected to applied forces of densities $\tilde{\boldsymbol{f}}: \tilde{\Omega} \rightarrow \mathbb{R}^{3}$ and $\tilde{\boldsymbol{g}}: \tilde{\Gamma}_{1} \rightarrow \mathbb{R}^{3}$ is in equilibrium if there exists a vector field

$$
\tilde{\boldsymbol{t}}:\{\tilde{\Omega}\}^{-} \times S_{2} \rightarrow \mathbb{R}^{3}
$$

such that, for all domains $\tilde{A} \subset \tilde{\Omega}$,

$$
\begin{aligned}
& \int_{\tilde{A}} \tilde{\boldsymbol{f}} d \tilde{x}+\int_{\partial \tilde{A}} \tilde{\boldsymbol{t}}(\tilde{x}, \tilde{\boldsymbol{n}}(\tilde{x})) d \tilde{a}=\mathbf{0}, \\
& \int_{\tilde{A}} \tilde{\boldsymbol{x}} \wedge \tilde{\boldsymbol{f}} d \tilde{x}+\int_{\partial \tilde{A}} \tilde{\boldsymbol{x}} \wedge \tilde{\boldsymbol{t}}(\tilde{x}, \tilde{\boldsymbol{n}}(\tilde{x})) d \tilde{a}=\mathbf{0}, \\
& \tilde{\boldsymbol{t}}(\tilde{x}, \tilde{\boldsymbol{n}}(\tilde{x}))=\tilde{\boldsymbol{g}}(\tilde{x}) \text { for } \partial \Omega \text {-almost all } \tilde{x} \in \partial \tilde{A} \cap \tilde{\Gamma}_{1},
\end{aligned}
$$

where $\tilde{\boldsymbol{n}}(\tilde{x})$ denotes the exterior unit normal vector at $\tilde{x}$ to the surface $\partial \tilde{A}$ (because $\tilde{A}$ is a domain, $\tilde{\boldsymbol{n}}(\tilde{x})$ exists for $d \tilde{x}$-almost all $\tilde{x} \in \partial \tilde{A})$.

This axiom postulates in effect that the "equilibrium" of the body to the applied forces is reflected by the existence of a vector field $\tilde{\boldsymbol{t}}$ that depends only on the two variables $\tilde{x}$ and $\tilde{\boldsymbol{n}}(\tilde{x})$. 
The following theorem, which is due to Cauchy, shows that the dependence of $\tilde{\boldsymbol{t}}$ on the second variable is necessarily linear:

Theorem 1.2-1. If $\tilde{\boldsymbol{t}}(\cdot, \tilde{\boldsymbol{n}}):\{\tilde{\Omega}\}^{-} \rightarrow \mathbb{R}^{3}$ is of class $\mathcal{C}^{1}$ for all $\tilde{\boldsymbol{n}} \in S_{2}, \tilde{\boldsymbol{t}}(\tilde{x}, \cdot)$ : $S_{2} \rightarrow \mathbb{R}^{3}$ is continuous for all $\tilde{x} \in\{\tilde{\Omega}\}^{-}$, and $\tilde{\boldsymbol{f}}:\{\tilde{\Omega}\}^{-} \rightarrow \mathbb{R}^{3}$ is continuous, then $\tilde{\boldsymbol{t}}:\{\tilde{\Omega}\}^{-} \times S_{2} \rightarrow \mathbb{R}^{3}$ is linear with respect to the second variable.

Proof. The proof consists in applying the stress principle to particular subdomains in $\{\tilde{\Omega}\}^{-}$. For details, see, e.g., Ciarlet [18] or Gurtin \& Martins [55].

In other words, there exists a matrix field $\tilde{\boldsymbol{T}}:\{\tilde{\Omega}\}^{-} \rightarrow \mathbb{M}^{3}$ of class $\mathcal{C}^{1}$ such that

$$
\tilde{\boldsymbol{t}}(\tilde{x}, \tilde{\boldsymbol{n}})=\tilde{\boldsymbol{T}}(\tilde{x}) \tilde{\boldsymbol{n}} \text { for all } \tilde{x} \in\{\tilde{\Omega}\}^{-} \text {and all } \tilde{\boldsymbol{n}} \in S_{2} .
$$

Combining Cauchy's theorem with the stress principle of Euler and Cauchy yields, by means of Stokes' formula (see Section 1.1), the following equations of equilibrium in the deformed configuration:

Theorem 1.2-2. The matrix field $\tilde{\boldsymbol{T}}:\{\tilde{\Omega}\}^{-} \rightarrow \mathbb{M}^{3}$ satisfies

$$
\begin{array}{r}
-\operatorname{div} \tilde{\boldsymbol{T}}(\tilde{x})=\tilde{\boldsymbol{f}}(\tilde{x}) \text { for all } \tilde{x} \in \tilde{\Omega}, \\
\tilde{\boldsymbol{T}}(\tilde{x}) \tilde{\boldsymbol{n}}(\tilde{x})=\tilde{\boldsymbol{g}}(\tilde{x}) \text { for all } \tilde{x} \in \tilde{\Gamma}_{1}, \\
\tilde{\boldsymbol{T}}(\tilde{x}) \in \mathbb{S}^{3} \text { for all } \tilde{x} \in \tilde{\Omega} .
\end{array}
$$

The system (1.2-1) is defined over the deformed configuration $\tilde{\Omega}$, which is unknown. Fortunately, it can be conveniently reformulated in terms of functions defined over the reference configurence $\Omega$ of the body, which is known. To this end, we use the change of variables $\tilde{x}=\boldsymbol{\Phi}(x)$ defined by the unknown deformation $\Phi: \bar{\Omega} \rightarrow\{\tilde{\Omega}\}^{-}$, assumed to be injective, and the following formulas between the volume and area elements in $\{\tilde{\Omega}\}^{-}$and $\bar{\Omega}$ (with self-explanatory notations)

$$
\begin{aligned}
& d \tilde{x}=|\operatorname{det} \boldsymbol{\nabla} \boldsymbol{\Phi}(x)| d x, \\
& \tilde{\boldsymbol{n}}(\tilde{x}) d \tilde{a}=\mathbf{C o f} \boldsymbol{\nabla} \boldsymbol{\Phi}(x) \boldsymbol{n}(x) d a .
\end{aligned}
$$

We also define the vector fields $\boldsymbol{f}: \Omega \rightarrow \mathbb{R}^{3}$ and $\boldsymbol{g}: \Gamma_{1} \rightarrow \mathbb{R}^{3}$ by

$$
\begin{aligned}
& \tilde{\boldsymbol{f}}(\tilde{x}) d \tilde{x}=\boldsymbol{f}(x) d x, \\
& \tilde{\boldsymbol{g}}(\tilde{x}) d \tilde{a}=\boldsymbol{g}(x) d a .
\end{aligned}
$$

Note that, like the fields $\tilde{\boldsymbol{f}}$ and $\tilde{\boldsymbol{g}}$, the fields $\boldsymbol{f}$ and $\boldsymbol{g}$ may, or may not, depend on the unknown deformation $\boldsymbol{\Phi}$.

First of all, assuming that $\boldsymbol{\Phi}$ is smooth enough and using the change of variables $\Phi: \bar{\Omega} \rightarrow\{\tilde{\Omega}\}^{-}$in the first equation of (1.2-1), we deduce that, for all domains $A \subset \Omega$,

$$
\int_{A} \boldsymbol{f}(x) d x+\int_{\partial A} \tilde{\boldsymbol{T}}(\boldsymbol{\Phi}(x)) \operatorname{Cof} \boldsymbol{\nabla} \boldsymbol{\Phi}(x) \boldsymbol{n}(x) d a=\mathbf{0} .
$$

The matrix field $\boldsymbol{T}: \bar{\Omega} \rightarrow \mathbb{M}^{3}$ appearing in the second integral, viz., that defined by

$$
\boldsymbol{T}(x):=\tilde{\boldsymbol{T}}(\boldsymbol{\Phi}(x)) \operatorname{Cof} \nabla \boldsymbol{\Phi}(x) \text { for all } x \in \bar{\Omega},
$$

is called the first Piola-Kirchhoff stress tensor field. In terms of this tensor, the above relation read

$$
\int_{A} \boldsymbol{f}(x) d x+\int_{\partial A} \boldsymbol{T}(x) \boldsymbol{n}(x) d a=\mathbf{0}
$$


which implies that the matrix field $\boldsymbol{T}$ satisfies the following partial differential equation:

$$
-\operatorname{div} \boldsymbol{T}(x)=\boldsymbol{f}(x) \text { for all } x \in \Omega \text {. }
$$

Using the identity

$$
\boldsymbol{\nabla} \boldsymbol{\Phi}(x)^{-1} \boldsymbol{T}(x)=\boldsymbol{\nabla} \boldsymbol{\Phi}(x)^{-1}[\operatorname{det} \boldsymbol{\nabla} \boldsymbol{\Phi}(x) \tilde{\boldsymbol{T}}(\boldsymbol{\Phi}(x))] \boldsymbol{\nabla} \boldsymbol{\Phi}(x)^{-T},
$$

which follows from the definition of $\boldsymbol{T}(x)$ and from the expression of the inverse of a matrix in terms of its cofactor matrix, we furthermore deduce from the symmetry of the matrix $\tilde{\boldsymbol{T}}(\tilde{x})$ that the matrix $\left(\boldsymbol{\nabla} \boldsymbol{\Phi}(x)^{-1} \boldsymbol{T}(x)\right)$ is also symmetric.

It is then clear that the equations of equilibrium in the deformed configuration (see eqns. (1.2-1)) are equivalent with the following equations of equilibrium in the reference configuration:

$$
\begin{aligned}
& -\operatorname{div} \boldsymbol{T}(x)=\boldsymbol{f}(x) \quad \text { for all } x \in \Omega, \\
& \boldsymbol{T}(x) \boldsymbol{n}(x)=\boldsymbol{g}(x) \quad \text { for all } x \in \Gamma_{1}, \\
& \boldsymbol{\nabla} \boldsymbol{\Phi}(x)^{-1} \boldsymbol{T}(x) \in \mathbb{S}^{3} \quad \text { for all } x \in \Omega,
\end{aligned}
$$

where the subset $\Gamma_{1}$ of $\partial \Omega$ is defined by $\tilde{\Gamma}_{1}=\boldsymbol{\Phi}\left(\Gamma_{1}\right)$.

Finally, let the second Piola-Kirchhoff stress tensor field $\Sigma: \bar{\Omega} \rightarrow \mathbb{S}^{3}$ be defined by

$$
\boldsymbol{\Sigma}(x):=\boldsymbol{\nabla} \boldsymbol{\Phi}(x)^{-1} \boldsymbol{T}(x) \text { for all } x \in \Omega .
$$

Then the equations of equilibrium defined in the reference configuration take the equivalent form

$$
\begin{aligned}
-\operatorname{div}(\boldsymbol{\nabla} \boldsymbol{\Phi}(x) \boldsymbol{\Sigma}(x)) & =\boldsymbol{f}(x) \text { for all } x \in \Omega, \\
(\boldsymbol{\nabla} \boldsymbol{\Phi}(x) \boldsymbol{\Sigma}(x)) \boldsymbol{n}(x) & =\boldsymbol{g}(x) \text { for all } x \in \Gamma_{1},
\end{aligned}
$$

in terms of the symmetric tensor field $\boldsymbol{\Sigma}$.

The unknowns in either system of equations of equilibrium are the deformation of the body defined by the vector field $\boldsymbol{\Phi}: \bar{\Omega} \rightarrow \mathbb{R}^{3}$, and the stress field inside the body defined by the fields $\boldsymbol{T}: \bar{\Omega} \rightarrow \mathbb{M}^{3}$ or $\boldsymbol{\Sigma}: \bar{\Omega} \rightarrow \mathbb{S}^{3}$. In order to determine these unknowns, either system (1.2-2) or (1.2-3) has to be supplemented with an equation relating these fields. This is the object of the next section.

\subsection{Constitutive Equations of Elastic materials}

It is clear that the stress tensor field should depend on the deformation induced by the applied forces. This dependence is reflected by the constitutive equation of the material, by means of a response function, specific to the material considered. In this article, we will consider one class of such materials, according to the following definition: A material is elastic if there exists a function $\boldsymbol{T}^{\sharp}: \bar{\Omega} \times \mathbb{M}_{+}^{3} \rightarrow \mathbb{M}^{3}$ such that

$$
\boldsymbol{T}(x)=\boldsymbol{T}^{\sharp}(x, \boldsymbol{\nabla} \boldsymbol{\Phi}(x)) \text { for all } x \in \bar{\Omega} .
$$

Equivalently, a material is elastic if there exists a function $\Sigma^{\sharp}: \bar{\Omega} \times \mathbb{M}_{+}^{3} \rightarrow \mathbb{S}^{3}$ such that

$$
\boldsymbol{\Sigma}(x)=\boldsymbol{\Sigma}^{\sharp}(x, \boldsymbol{\nabla} \boldsymbol{\Phi}(x)) \text { for all } x \in \bar{\Omega} .
$$

Either function $\boldsymbol{T}^{\sharp}$ or $\boldsymbol{\Sigma}^{\sharp}$ is called the response function of the material.

$A$ response function cannot be arbitrary, because a general axiom in physics asserts that any "observable quantity" must be independent of the particular orthogonal basis in which it is computed. For an elastic material, the "observable 
quantity" computed through a constitutive equation is the stress vector field $\tilde{\boldsymbol{t}}$. Therefore this vector field must be independent of the particular orthogonal basis in which it is computed. This property, which must be satisfied by all elastic materials, is called the axiom of material frame-indifference. The following theorem translates this axiom in terms of the response function of the material.

Theorem 1.3-1. An elastic material satisfies the axiom of material frame-indifference if and only if

$$
\boldsymbol{T}^{\sharp}(x, \boldsymbol{Q F})=\boldsymbol{Q} \boldsymbol{T}^{\sharp}(x, \boldsymbol{F}) \text { for all } x \in \bar{\Omega} \text { and } \boldsymbol{Q} \in \mathbb{O}_{+}^{3} \text { and } \boldsymbol{F} \in \mathbb{M}_{+}^{3},
$$

or equivalently, if and only if

$$
\boldsymbol{\Sigma}^{\sharp}(x, \boldsymbol{Q F})=\boldsymbol{\Sigma}^{\sharp}(x, \boldsymbol{F}) \text { for all } x \in \bar{\Omega} \text { and } \boldsymbol{Q} \in \mathbb{O}_{+}^{3} \text { and } \boldsymbol{F} \in \mathbb{M}_{+}^{3} .
$$

The second equivalence implies that the response function $\boldsymbol{\Sigma}^{\sharp}$ depend on $\boldsymbol{F}$ only via the symmetric positive definite matrix $\boldsymbol{U}:=\left(\boldsymbol{F}^{T} \boldsymbol{F}\right)^{1 / 2}$, the square root of the symmetric positive definite matrix $\left(\boldsymbol{F}^{T} \boldsymbol{F}\right) \in \mathbb{S}_{>}^{3}$. To see this, one uses the polar factorisation $\boldsymbol{F}=\boldsymbol{R U}$, where $\boldsymbol{R}:=\boldsymbol{F} \boldsymbol{U}^{-1} \in \mathbb{O}_{+}^{3}$, in the second equivalence of Theorem 1.3-1 to deduce that

$$
\boldsymbol{\Sigma}^{\sharp}(x, \boldsymbol{F})=\boldsymbol{\Sigma}^{\sharp}(x, \boldsymbol{U}) \text { for all } x \in \bar{\Omega} \text { and } \boldsymbol{F}=\boldsymbol{R U} \in \mathbb{M}_{+}^{3} .
$$

This implies that the second Piola-Kirchhoff stress tensor field $\boldsymbol{\Sigma}: \bar{\Omega} \rightarrow \mathbb{S}^{3}$ depends on the deformation field $\boldsymbol{\Phi}: \bar{\Omega} \rightarrow \mathbb{R}^{3}$ only via the associated metric tensor field $C:=\nabla \Phi^{T} \nabla \Phi$, i.e.,

$$
\boldsymbol{\Sigma}(x)=\tilde{\boldsymbol{\Sigma}}(x, \boldsymbol{C}(x)) \text { for all } x \in \bar{\Omega},
$$

where the function $\tilde{\boldsymbol{\Sigma}}: \bar{\Omega} \times \mathbb{S}_{>}^{3} \rightarrow \mathbb{S}^{3}$ is defined by

$$
\tilde{\boldsymbol{\Sigma}}(x, \boldsymbol{C}):=\boldsymbol{\Sigma}^{\sharp}\left(x, \boldsymbol{C}^{1 / 2}\right) \text { for all } x \in \bar{\Omega} \text { and } \boldsymbol{C} \in \mathbb{S}_{>}^{3} .
$$

We just saw how the axiom of material frame-indifference restricts the form of the response function. We now examine how its form can be further restricted by other properties that a given material may possess.

An elastic material is isotropic at a point $x$ of the reference configuration if the response of the material "is the same in all directions", i.e., if the Cauchy stress tensor $\tilde{\boldsymbol{T}}(\tilde{x})$ is the same if the reference configuration is rotated by an arbitrary matrix of $\mathbb{O}_{+}^{3}$ around the point $x$. An elastic material occupying a reference configuration $\bar{\Omega}$ is isotropic if it is isotropic at all points of $\bar{\Omega}$. The following theorem gives a characterisation of the response function of an isotropic elastic material:

Theorem 1.3-2. An elastic material occupying a reference configuration $\bar{\Omega}$ is isotropic if and only if

$$
\boldsymbol{T}^{\sharp}(x, \boldsymbol{F} \boldsymbol{Q})=\boldsymbol{T}^{\sharp}(x, \boldsymbol{F}) \boldsymbol{Q} \text { for all } x \in \bar{\Omega} \text { and } \boldsymbol{Q} \in \mathbb{O}_{+}^{3} \text { and } \boldsymbol{F} \in \mathbb{M}_{+}^{3},
$$

or equivalently, if and only if

$$
\boldsymbol{\Sigma}^{\sharp}(x, \boldsymbol{F} \boldsymbol{Q})=\boldsymbol{Q}^{T} \boldsymbol{\Sigma}^{\sharp}(x, \boldsymbol{F}) \boldsymbol{Q} \text { for all } x \in \bar{\Omega} \text { and } \boldsymbol{Q} \in \mathbb{O}_{+}^{3} \text { and } \boldsymbol{F} \in \mathbb{M}_{+}^{3} .
$$

Another property that an elastic material may satisfy is the property of homogeneity: An elastic material occupying a reference configuration $\bar{\Omega}$ is homogeneous if its response function is independent of the particular point $x \in \bar{\Omega}$ considered. This means that the response function $T^{\sharp}: \bar{\Omega} \times \mathbb{M}_{+}^{3} \rightarrow \mathbb{M}^{3}$, or equivalently the response 
function $\boldsymbol{\Sigma}^{\sharp}: \bar{\Omega} \times \mathbb{M}_{+}^{3} \rightarrow \mathbb{S}^{3}$, does not depend on the first variable. In other words, there exist mappings (still denoted) $\boldsymbol{T}^{\sharp}: \mathbb{M}_{+}^{3} \rightarrow \mathbb{M}^{3}$ and $\boldsymbol{\Sigma}^{\sharp}: \mathbb{M}_{+}^{3} \rightarrow \mathbb{S}^{3}$ such that

$$
\boldsymbol{T}^{\sharp}(x, \boldsymbol{F})=\boldsymbol{T}^{\sharp}(\boldsymbol{F}) \text { for all } x \in \bar{\Omega} \text { and } \boldsymbol{F} \in \mathbb{M}_{+}^{3} \text {, }
$$

and

$$
\boldsymbol{\Sigma}^{\sharp}(x, \boldsymbol{F})=\boldsymbol{\Sigma}^{\sharp}(\boldsymbol{F}) \text { for all } x \in \bar{\Omega} \text { and } \boldsymbol{F} \in \mathbb{M}_{+}^{3} .
$$

The response function of an elastic material can be further restricted if its reference configuration is a natural state, according to the following definition: A reference configuration $\bar{\Omega}$ is called a natural state, or equivalently is said to be stress-free, if

$$
\boldsymbol{T}^{\sharp}(x, \boldsymbol{I})=\mathbf{0} \text { for all } x \in \bar{\Omega},
$$

or equivalently, if

$$
\boldsymbol{\Sigma}^{\sharp}(x, \boldsymbol{I})=\mathbf{0} \text { for all } x \in \bar{\Omega} .
$$

We have seen that the second Piola-Kirchhoff stress tensor field $\boldsymbol{\Sigma}: \bar{\Omega} \rightarrow \mathbb{S}^{3}$ is expressed in terms of the deformation field $\Phi: \bar{\Omega} \rightarrow \mathbb{R}^{3}$ as

$$
\boldsymbol{\Sigma}(x)=\tilde{\boldsymbol{\Sigma}}(x, \boldsymbol{C}(x)) \text {, where } \boldsymbol{C}(x)=\boldsymbol{\nabla} \boldsymbol{\Phi}^{T}(x) \boldsymbol{\nabla} \boldsymbol{\Phi}(x) \text { for all } x \in \bar{\Omega} .
$$

If the elastic material is isotropic, then the dependence of $\boldsymbol{\Sigma}(x)$ in terms of $\boldsymbol{C}(x)$ can be further reduced in a remarkable way, according to the following RivlinEricksen theorem:

Theorem 1.3-3. If an elastic material is isotropic and satisfies the principle of material frame-indifference, then there exists functions $\gamma_{i}^{\sharp}: \Omega \times \mathbb{R}^{3} \rightarrow \mathbb{R}, i \in$ $\{1,2,3\}$, such that

$$
\boldsymbol{\Sigma}(x)=\gamma_{0}(x) \boldsymbol{I}+\gamma_{1}(x) \boldsymbol{C}(x)+\gamma_{2}(x) \boldsymbol{C}^{2}(x) \text { for all } x \in \bar{\Omega},
$$

where $\gamma_{i}(x)=\gamma_{i}^{\sharp}(x, \operatorname{tr} \boldsymbol{C}, \operatorname{tr}(\mathbf{C o f} \boldsymbol{C}), \operatorname{det} \boldsymbol{C})$.

Proof. See Rivlin \& Ericksen [74] or Ciarlet [18].

Note that the numbers $\operatorname{tr} \boldsymbol{C}(x), \operatorname{tr}(\mathbf{C o f} \boldsymbol{C}(x))$, and $\operatorname{det} \boldsymbol{C}(x)$ appearing in the above theorem constitute the three principal invariants of the matrix $\boldsymbol{C}(x)$.

Although the Rivlin-Ericksen theorem substantially reduces the range of possible response functions of elastic materials that are isotropic and satisfy the principle of frame-indifference, the expression of $\boldsymbol{\Sigma}$ is still far too general in view of an effective resolution of the equilibrium equations. To further simplify this expression, we now restrict ourselves to deformations that are "close to the identity".

In terms of the displacement filed $\boldsymbol{u}: \Omega \rightarrow \mathbb{R}^{3}$, which is related to the deformation $\Phi: \Omega \rightarrow \mathbb{R}^{3}$ by the formula

$$
\boldsymbol{\Phi}(x)=x+\boldsymbol{u}(x) \text { for all } x \in \bar{\Omega},
$$

the metric tensor field $\boldsymbol{C}$ has the expression

$$
\boldsymbol{C}(x)=\boldsymbol{I}+2 \boldsymbol{E}(x),
$$

where

$$
\boldsymbol{E}(x):=\frac{1}{2}\left(\boldsymbol{\nabla} \boldsymbol{u}^{T}(x)+\boldsymbol{\nabla} \boldsymbol{u}(x)+\boldsymbol{\nabla} \boldsymbol{u}^{T}(x) \boldsymbol{\nabla} \boldsymbol{u}(x)\right)
$$

denotes the Green-St Venant strain tensor at $x$. 
Thanks to the above assumption on the deformation, the matrices $\boldsymbol{E}(x)$ are "small" for all $x \in \bar{\Omega}$, and therefore one can use Taylor expansions to further simplify the expression of the response function given by the Rivlin-Ericksen theorem. Specifically, using the Taylor expansions

$$
\begin{aligned}
\operatorname{tr} \boldsymbol{C}(x) & =3+2 \operatorname{tr} \boldsymbol{E}(x), \\
\operatorname{tr}(\mathbf{C o f} \boldsymbol{C}(x)) & =3+4 \operatorname{tr} \boldsymbol{E}(x)+o(|\boldsymbol{E}(x)|), \\
\operatorname{det} \boldsymbol{C}(x) & =1+2 \operatorname{tr} \boldsymbol{E}(x)+o(|\boldsymbol{E}(x)|), \\
\boldsymbol{C}^{2}(x) & =1+4 \boldsymbol{E}(x)+o(|\boldsymbol{E}(x)|),
\end{aligned}
$$

and assuming that the functions $\gamma_{i}^{\sharp}$ are smooth enough, we deduce from the RivlinEricksen theorem that

$$
\boldsymbol{\Sigma}(x)=\boldsymbol{\Sigma}^{\sharp}(x, \boldsymbol{I})+\{(\lambda(x) \operatorname{tr} \boldsymbol{E}(x)) \boldsymbol{I}+2 \mu(x) \boldsymbol{E}(x)\}+o_{x}(|\boldsymbol{E}(x)|),
$$

where the real-valued functions $\lambda(x)$ and $\mu(x)$ are independent of the displacement field $\boldsymbol{u}$. If in addition the material is homogeneous, then $\lambda$ and $\mu$ are constants.

To sum up, the constitutive equation of a homogeneous and isotropic elastic material that satisfies the axiom of frame-indifference must be such that

$$
\boldsymbol{\Sigma}(x)=\boldsymbol{\Sigma}^{\sharp}(x, \boldsymbol{I})+\lambda(\operatorname{tr} \boldsymbol{E}(x)) \boldsymbol{I}+2 \mu \boldsymbol{E}(x)+o_{x}(|\boldsymbol{E}(x)|) \text { for all } x \in \bar{\Omega} .
$$

If in addition $\Omega$ is a natural state, a natural candidate for a constitutive equation is thus

$$
\boldsymbol{\Sigma}(x)=\lambda(\operatorname{tr} \boldsymbol{E}(x)) \boldsymbol{I}+2 \mu \boldsymbol{E}(x) \text { for all } x \in \bar{\Omega},
$$

and in this case $\lambda$ and $\mu$ are then called the Lamé constants of the material.

A material whose constitutive equation has the above expression is called a $\mathbf{S t}$ Venant-Kirchhoff material. Note that the constitutive equation of a St VenantKirchhoff material is invertible, in the sense that the field $\boldsymbol{E}$ can be also expressed as a function of the field $\boldsymbol{\Sigma}$ as

$$
\boldsymbol{E}(x)=\frac{1}{2 \mu} \boldsymbol{\Sigma}(x)-\frac{\nu}{E}(\operatorname{tr} \boldsymbol{\Sigma}(x)) \boldsymbol{I} \text { for all } x \in \bar{\Omega} .
$$

Remark. The Lamé constants are determined experimentally for each elastic material and experimental evidence shows that they are both strictly positive (for instance, $\lambda=10^{6} \mathrm{~kg} / \mathrm{cm}^{2}$ and $\mu=820000 \mathrm{~kg} / \mathrm{cm}^{2}$ for steel; $\lambda=40000 \mathrm{~kg} / \mathrm{cm}^{2}$ and $\mu=1200 \mathrm{~kg} / \mathrm{cm}^{2}$ for rubber). Their explicit values do not play any rôle in our subsequent analysis; only their positivity will be used. The Lamé coefficients are sometimes expressed in terms of the Poisson coefficient $\nu$ and Young modulus E through the expressions

$$
\nu=\frac{\lambda}{2(\lambda+\mu)} \text { and } E=\frac{\mu(3 \lambda+2 \mu)}{\lambda+\mu} .
$$

\subsection{The Equations of NONLINEAR AND LineARIZED THREE-Dimensional ELASTICITY}

It remains to combine the equations of equilibrium (equations (1.2-3) in Section 1.2) with the constitutive equation of the material considered (Section 1.3) and with boundary conditions on $\Gamma_{0}:=\partial \Omega \backslash \Gamma_{1}$. Assuming that the constituting material 
has a known response function given by $\boldsymbol{T}^{\sharp}$ or by $\boldsymbol{\Sigma}^{\sharp}$ and that the body is held fixed on $\Gamma_{0}$, we conclude in this fashion that the deformation arising in the body in response to the applied forces of densities $\boldsymbol{f}$ and $\boldsymbol{g}$ satisfies the nonlinear boundary value problem:

$$
\begin{aligned}
-\operatorname{div} \boldsymbol{T}(x) & =\boldsymbol{f}(x), & & x \in \Omega, \\
\boldsymbol{\Phi}(x) & =x, & & x \in \Gamma_{0}, \\
\boldsymbol{T}(x) \boldsymbol{n}(x) & =\boldsymbol{g}(x), & & x \in \Gamma_{1},
\end{aligned}
$$

where

$$
\boldsymbol{T}(x)=\boldsymbol{T}^{\sharp}(x, \boldsymbol{\nabla} \boldsymbol{\Phi}(x))=\boldsymbol{\nabla} \boldsymbol{\Phi}(x) \boldsymbol{\Sigma}^{\sharp}(x, \boldsymbol{\nabla} \boldsymbol{\Phi}(x)) \text { for all } x \in \bar{\Omega} .
$$

The equations (1.4-1) constitute the equations of nonlinear three-dimensional elasticity. We will give in Sections 1.7 and 1.8 various sets of assumptions guaranteeing that this problem has solutions.

Consider a body made of an isotropic and homogeneous elastic material such that its reference configuration is a natural state, so that its constitutive equation is (see Section 1.3):

$$
\boldsymbol{\Sigma}(x)=\lambda(\operatorname{tr} \boldsymbol{E}(x)) \boldsymbol{I}+2 \mu \boldsymbol{E}(x)+o(|\boldsymbol{E}(x)|),
$$

where $\lambda>0$ and $\mu>0$ are the Lamé constants of the material. The equations of linearized three-dimensional elasticity are obtained from the above nonlinear equations under the assumption that the body will undergo a "small" displacement, in the sense that

$$
\boldsymbol{\Phi}(x)=x+\boldsymbol{u}(x) \text { with }|\boldsymbol{\nabla} \boldsymbol{u}(x)| \ll 1 \text { for all } x \in \bar{\Omega} .
$$

Then, for all $x \in \bar{\Omega}$,

$$
\boldsymbol{E}(x)=\frac{1}{2}\left(\boldsymbol{\nabla} \boldsymbol{\Phi}^{T}(x) \boldsymbol{\nabla} \boldsymbol{\Phi}(x)-\boldsymbol{I}\right)=\frac{1}{2}\left(\boldsymbol{\nabla} \boldsymbol{u}^{T}(x)+\boldsymbol{\nabla} \boldsymbol{u}(x)\right)+o_{x}(|\boldsymbol{\nabla} \boldsymbol{u}(x)|),
$$

and

$$
\begin{aligned}
\boldsymbol{T}(x) & =\boldsymbol{\nabla} \boldsymbol{\Phi}(x) \boldsymbol{\Sigma}(x)=(\boldsymbol{I}+\boldsymbol{\nabla} \boldsymbol{u}(x))(\lambda(\operatorname{tr} \boldsymbol{E}(x)) \boldsymbol{I}+2 \mu \boldsymbol{E}(x)) \\
& =\frac{\lambda}{2} \operatorname{tr}\left(\boldsymbol{\nabla} \boldsymbol{u}^{T}(x)+\boldsymbol{\nabla} \boldsymbol{u}(x)\right)+\mu\left(\boldsymbol{\nabla} \boldsymbol{u}^{T}(x)+\boldsymbol{\nabla} \boldsymbol{u}(x)\right)+o_{x}(|\boldsymbol{\nabla} \boldsymbol{u}(x)|) .
\end{aligned}
$$

Therefore the equations of linearized three-dimensional elasticity, which are obtained from (1.4-1) by replacing $\boldsymbol{T}(x)$ by its linear part with respect to $\boldsymbol{\nabla} \boldsymbol{u}(x)$, are given by

$$
\begin{aligned}
-\operatorname{div} \boldsymbol{\sigma}(x) & =\boldsymbol{f}(x), & & x \in \Omega, \\
\boldsymbol{u}(x) & =\mathbf{0}, & & x \in \Gamma_{0}, \\
\boldsymbol{\sigma}(x) \boldsymbol{n}(x) & =\boldsymbol{g}(x), & & x \in \Gamma_{1},
\end{aligned}
$$

where

$$
\boldsymbol{\sigma}(x)=\lambda(\operatorname{tr} \mathbf{e}(x)) \boldsymbol{I}+2 \mu \mathbf{e}(x) \text { and } \mathbf{e}(x)=\frac{1}{2}\left(\boldsymbol{\nabla} \boldsymbol{u}^{T}(x)+\boldsymbol{\nabla} \boldsymbol{u}(x)\right) .
$$

We show in the next section that this linear system has a unique solution in appropriate function spaces. 


\subsection{A fundamental Lemma of J.L. Lions}

We first review some essential definitions and notations, together with a fundamental lemma of J.L. Lions (Theorem 1.5-1). This lemma will play a key rôle in the proofs of Korn's inequality in the next Section.

Let $\Omega$ be a domain in $\mathbb{R}^{n}$. We recall that, for each integer $m \geq 1, H^{m}(\Omega)$ and $H_{0}^{m}(\Omega)$ denote the usual Sobolev spaces. In particular,

$$
\begin{aligned}
& H^{1}(\Omega):=\left\{v \in L^{2}(\Omega) ; \partial_{i} v \in L^{2}(\Omega), 1 \leq i \leq n\right\}, \\
& H^{2}(\Omega):=\left\{v \in H^{1}(\Omega) ; \partial_{i j} v \in L^{2}(\Omega), 1 \leq i, j \leq n\right\},
\end{aligned}
$$

where $\partial_{i} v$ and $\partial_{i j} v$ denote partial derivatives in the sense of distributions, and

$$
H_{0}^{1}(\Omega):=\left\{v \in H^{1}(\Omega) ; v=0 \text { on } \Gamma\right\},
$$

where the relation $v=0$ on $\Gamma$ is to be understood in the sense of trace. The norm in $L^{2}(\Omega)$ is noted $\|\cdot\|_{L^{2}(\Omega)}$ and the norm in $H^{m}(\Omega), m \geq 1$, is noted $\|\cdot\|_{H^{m}(\Omega)}$. In particular then,

$$
\begin{aligned}
& \|v\|_{L^{2}(\Omega)}:=\left\{\int_{\Omega}|v|^{2} d x\right\}^{1 / 2} \text { if } v \in L^{2}(\Omega), \\
& \|v\|_{H^{1}(\Omega)}:=\left\{\|v\|_{L^{2}(\Omega)}^{2}+\sum_{i=1}^{n}\left\|\partial_{i} v\right\|_{L^{2}(\Omega)}^{2}\right\}^{1 / 2} \text { if } v \in H^{1}(\Omega) .
\end{aligned}
$$

We also consider the Sobolev space

$$
H^{-1}(\Omega):=\text { dual space of } H_{0}^{1}(\Omega) .
$$

Another possible definition of the space $H_{0}^{1}(\Omega)$ being

$$
H_{0}^{1}(\Omega)=\text { closure of } \mathcal{D}(\Omega) \text { with respect to }\|\cdot\|_{H^{1}(\Omega)},
$$

where $\mathcal{D}(\Omega)$ denotes the space of infinitely differentiable real-valued functions defined over $\Omega$ whose support is a compact subset of $\Omega$, it is clear that

$$
v \in L^{2}(\Omega) \Longrightarrow v \in H^{-1}(\Omega) \text { and } \partial_{i} v \in H^{-1}(\Omega), 1 \leq i \leq n,
$$

since (the duality between the spaces $\mathcal{D}(\Omega)$ and $\mathcal{D}^{\prime}(\Omega)$ is denoted by $\langle\cdot, \cdot\rangle$ ):

$$
\begin{array}{r}
|\langle v, \varphi\rangle|=\left|\int_{\Omega} v \varphi d x\right| \leq\|v\|_{L^{2}(\Omega)}\|\varphi\|_{H^{1}(\Omega)}, \\
\left|\left\langle\partial_{i} v, \varphi\right\rangle\right|=\left|-\left\langle v, \partial_{i} \varphi\right\rangle\right|=\left|-\int_{\Omega} v \partial_{i} \varphi d x\right| \leq\|v\|_{L^{2}(\Omega)}\|\varphi\|_{H^{1}(\Omega)}
\end{array}
$$

for all $\varphi \in \mathcal{D}(\Omega)$. It is remarkable (but also remarkably difficult to prove!) that the converse implication holds:

Theorem 1.5-1. Let $\Omega$ be a domain in $\mathbb{R}^{n}$ and let $v$ be a distribution on $\Omega$. Then

$$
\left\{v \in H^{-1}(\Omega) \text { and } \partial_{i} v \in H^{-1}(\Omega), 1 \leq i \leq n\right\} \Longrightarrow v \in L^{2}(\Omega) .
$$

This implication was first proved by J.L. Lions, as stated in Magenes \& Stampacchia [66], p. 320, Note $\left({ }^{27}\right)$; for this reason, it will be henceforth referred to as the lemma of J.L. Lions. Its first published proof for domains with smooth boundaries appeared in Duvaut \& Lions [46], p. 111; another proof was also given by Tartar [84]. Various extensions to "genuine" domains, i.e., with Lipschitzcontinuous boundaries, are given in Bolley \& Camus [14], Geymonat \& Suquet 
[52], and Borchers \& Sohr [15]; Amrouche \& Girault [6], Proposition 2.10 even proved that the more general implication

$$
\left\{v \in \mathcal{D}^{\prime}(\Omega) \text { and } \partial_{i} v \in H^{m}(\Omega), 1 \leq i \leq n\right\} \Longrightarrow v \in H^{m+1}(\Omega)
$$

holds for arbitrary integers $m \in \mathbb{Z}$.

\subsection{EXISTENCE THEORY IN LINEARIZED THREE-DIMENSIONAL ELASTICITY}

We define a weak solution to the equations of linearized three-dimensional elasticity (Section 1.4) as a solution to the variational equations

$$
\int_{\Omega} \boldsymbol{\sigma}: \boldsymbol{\nabla} \boldsymbol{v} d x=\int_{\Omega} \boldsymbol{f} \cdot \boldsymbol{v} d x+\int_{\Gamma_{1}} \boldsymbol{g} \cdot \boldsymbol{v} d a
$$

for all smooth vector fields $\boldsymbol{v}: \Omega \rightarrow \mathbb{R}^{3}$ that satisfy $\boldsymbol{v}=\mathbf{0}$ on $\Gamma_{0}$, where

$$
\boldsymbol{\sigma}=\lambda(\operatorname{tr} \mathbf{e}(\boldsymbol{u})) \boldsymbol{I}+2 \mu \mathbf{e}(\boldsymbol{u}) \text { and } \mathbf{e}(\boldsymbol{u})=\frac{1}{2}\left(\boldsymbol{\nabla} \boldsymbol{u}^{T}+\boldsymbol{\nabla} \boldsymbol{u}\right) .
$$

Note that, because the matrix field $\boldsymbol{\sigma}$ is symmetric, the integrand in the left-hand side can be also written as

$$
\sigma: \nabla v=\sigma: \mathbf{e}(v)
$$

where

$$
\mathbf{e}(\boldsymbol{v}):=\frac{1}{2}\left(\boldsymbol{\nabla} \boldsymbol{v}^{T}+\boldsymbol{\nabla} \boldsymbol{v}\right)
$$

The existence of a solution to the above variational problem follows from the Lax-Milgram lemma. In order to verify the hypotheses of this lemma, we first need to establish the following classical, and fundamental, inequality:

Theorem 1.6-1 (Korn's inequality). Let $\Omega$ be a domain in $\mathbb{R}^{3}$ and let $\Gamma_{0} \subset \partial \Omega$ be such that area $\Gamma_{0}>0$. Then there exists a constant $C$ such that

$$
\|\mathbf{e}(\boldsymbol{v})\|_{L^{2}\left(\Omega ; \mathbb{S}^{3}\right)} \geq C\|\boldsymbol{v}\|_{H^{1}\left(\Omega ; \mathbb{R}^{3}\right)}
$$

for all $\boldsymbol{v} \in H_{\Gamma_{0}}^{1}\left(\Omega ; \mathbb{R}^{3}\right):=\left\{\boldsymbol{v} \in H^{1}\left(\Omega ; \mathbb{R}^{3}\right) ; \boldsymbol{v}=\mathbf{0}\right.$ on $\left.\Gamma_{0}\right\}$.

Proof. Several proofs are available in the mathematical literature for this remarkable inequality. We adapt here that given in Duvaut \& Lions [46]. We proceed in several steps:

(i) Korn's inequality is a consequence of the identity

$$
\partial_{i j} v_{k}=\partial_{i} e_{j k}(\boldsymbol{v})+\partial_{j} e_{i k}(\boldsymbol{v})-\partial_{k} e_{i j}(\boldsymbol{v})
$$

relating the matrix fields $\boldsymbol{\nabla} \boldsymbol{v}=\left(\partial_{j} v_{i}\right)$ and $\mathbf{e}(\boldsymbol{v})=\left(e_{i j}(\boldsymbol{v})\right)$, where

$$
e_{i j}(\boldsymbol{v}):=\frac{1}{2}\left(\partial_{i} v_{j}+\partial_{j} v_{i}\right) .
$$

If $\boldsymbol{v} \in L^{2}\left(\Omega ; \mathbb{R}^{3}\right)$ and $\mathbf{e}(\boldsymbol{v}) \in L^{2}\left(\Omega ; \mathbb{S}^{3}\right)$, the above identity shows that $\partial_{i j} v_{k} \in$ $H^{-1}(\Omega)$. Since the functions $\partial_{j} v_{k}$ also belong to the space $H^{-1}(\Omega)$, the lemma of J.L. Lions (Theorem 1.5-1) shows that $\partial_{j} v_{k} \in L^{2}(\Omega)$. This implies that the space

$$
E\left(\Omega ; \mathbb{R}^{3}\right):=\left\{\boldsymbol{v} \in L^{2}\left(\Omega ; \mathbb{R}^{3}\right) ; \mathbf{e}(\boldsymbol{v}) \in L^{2}\left(\Omega ; \mathbb{S}^{3}\right)\right\}
$$

coincides with the Sobolev space $H^{1}\left(\Omega ; \mathbb{R}^{3}\right)$.

(ii) The space $E\left(\Omega ; \mathbb{R}^{3}\right)$, equipped with the norm

$$
\|\boldsymbol{v}\|_{E\left(\Omega ; \mathbb{R}^{3}\right)}:=\|\boldsymbol{v}\|_{L^{2}\left(\Omega ; \mathbb{R}^{3}\right)}+\|\mathbf{e}(\boldsymbol{v})\|_{L^{2}\left(\Omega ; \mathbb{R}^{3}\right)},
$$


is clearly a Hilbert space, as is the space $H^{1}\left(\Omega ; \mathbb{R}^{3}\right)$ equipped with the norm

$$
\|\boldsymbol{v}\|_{H^{1}\left(\Omega ; \mathbb{R}^{3}\right)}:=\|\boldsymbol{v}\|_{L^{2}\left(\Omega ; \mathbb{R}^{3}\right)}+\|\boldsymbol{\nabla} \boldsymbol{v}\|_{L^{2}\left(\Omega ; \mathbb{R}^{3}\right)} .
$$

Since the identity mapping

$$
\text { id : } \boldsymbol{v} \in H^{1}\left(\Omega ; \mathbb{R}^{3}\right) \mapsto \boldsymbol{v} \in E\left(\Omega ; \mathbb{R}^{3}\right)
$$

is clearly linear, bijective (thanks to the step (i)), and continuous, the open mapping theorem (see, e.g., Yosida [87]) shows that id is also an open mapping. Therefore, there exists a constant $C$ such that

$$
\|\boldsymbol{v}\|_{H^{1}\left(\Omega ; \mathbb{R}^{3}\right)} \leq C\|\boldsymbol{v}\|_{E\left(\Omega ; \mathbb{R}^{3}\right)} \text { for all } \boldsymbol{v} \in E\left(\Omega ; \mathbb{R}^{3}\right),
$$

or equivalently, such that

$$
\|\boldsymbol{v}\|_{L^{2}\left(\Omega ; \mathbb{R}^{3}\right)}+\|\mathbf{e}(\boldsymbol{v})\|_{L^{2}\left(\Omega ; \mathbb{S}^{3}\right)} \geq C^{-1}\|\boldsymbol{v}\|_{H^{1}\left(\Omega ; \mathbb{R}^{3}\right)}
$$

for all $\boldsymbol{v} \in H^{1}\left(\Omega ; \mathbb{R}^{3}\right)$.

(iii) We establish that, if $\boldsymbol{v} \in H_{\Gamma_{0}}^{1}\left(\Omega ; \mathbb{R}^{3}\right)$ satisfies $\mathbf{e}(\boldsymbol{v})=\mathbf{0}$, then $\boldsymbol{v}=\mathbf{0}$.

This is a consequence of the identity of Part (i), which shows that any field $\boldsymbol{v} \in H_{\Gamma_{0}}^{1}\left(\Omega ; \mathbb{R}^{3}\right)$ that satisfies $\mathbf{e}(\boldsymbol{v})=\mathbf{0}$ must also satisfy

$$
\partial_{i j} v_{k}=0 \text { in } \Omega \text {. }
$$

Therefore, by a classical result about distributions (see, e.g. Schwartz [80]), the field $\boldsymbol{v}$ must be affine, i.e., of the form $\boldsymbol{v}(x)=\boldsymbol{b}+\boldsymbol{A} \boldsymbol{x}$ for all $x \in \Omega$, where $\boldsymbol{b} \in \mathbb{R}^{3}$ and $\boldsymbol{A} \in \mathbb{M}^{3}$. Since the symmetric part of the gradient of $\boldsymbol{v}$, which is precisely $\mathbf{e}(\boldsymbol{v})$, vanishes in $\Omega$, the matrix $\boldsymbol{A}$ must be in addition antisymmetric. Since the rank of a nonzero antisymmetric matrix of order three is necessarily two, the locus of all points $x$ satisfying $\mathbf{a}+\boldsymbol{A x}=\mathbf{0}$ is either a line in $\mathbb{R}^{3}$ or an empty set, depending on whether the linear system $\mathbf{a}+\boldsymbol{A} \boldsymbol{x}=\mathbf{0}$ has solutions or not. But $\mathbf{a}+\boldsymbol{A} \boldsymbol{x}=\mathbf{0}$ on $\Gamma_{0}$ and area $\Gamma_{0}>0$. Hence $\boldsymbol{A}=\mathbf{0}$ and $\boldsymbol{b}=\mathbf{0}$, and thus $\boldsymbol{v}=\mathbf{0}$ in $\Omega$.

(iv) The Korn inequality of Theorem 1.6-1 then follows by contradiction. If the inequality were false, there would exist a sequence $\left(\boldsymbol{v}_{n}\right)_{n \in \mathbb{N}}$ in $H_{\Gamma_{0}}^{1}\left(\Omega ; \mathbb{R}^{3}\right)$ such that

$$
\begin{aligned}
& \left\|\boldsymbol{v}_{n}\right\|_{H^{1}\left(\Omega ; \mathbb{R}^{3}\right)}=1 \text { for all } n, \\
& \left\|\mathbf{e}\left(\boldsymbol{v}_{n}\right)\right\|_{L^{2}\left(\Omega ; \mathbb{S}^{3}\right)} \rightarrow 0 \text { as } n \rightarrow \infty .
\end{aligned}
$$

Because the set $\Omega$ is a domain, the inclusion $H^{1}\left(\Omega ; \mathbb{R}^{3}\right) \subset L^{2}\left(\Omega ; \mathbb{R}^{3}\right)$ is compact by the Rellich-Kondrasov theorem. The sequence $\left(\boldsymbol{v}_{n}\right)$ being bounded in $H^{1}\left(\Omega ; \mathbb{R}^{3}\right)$, it contains a subsequence $\left(\boldsymbol{v}_{\sigma(n)}\right)$, where $\sigma: \mathbb{N} \rightarrow \mathbb{N}$ is an increasing function, that converges in $L^{2}\left(\Omega ; \mathbb{R}^{3}\right)$ as $n \rightarrow \infty$.

Since the sequences $\left(\boldsymbol{v}_{\sigma(n)}\right)$ and $\left(\mathbf{e}\left(\boldsymbol{v}_{\sigma(n)}\right)\right)$ converge respectively in the spaces $L^{2}\left(\Omega ; \mathbb{R}^{3}\right)$ and $L^{2}\left(\Omega ; \mathbb{S}^{3}\right)$, they are Cauchy sequences in the same spaces. Therefore the sequence $\left(\boldsymbol{v}_{\sigma(n)}\right)$ is a Cauchy sequence with respect to the norm $\|\cdot\|_{E\left(\Omega ; \mathbb{R}^{3}\right)}$, hence with respect to the norm $\|\cdot\|_{H^{1}\left(\Omega ; \mathbb{R}^{3}\right)}$ by the inequality established in Part (ii).

The space $H_{\Gamma_{0}}^{1}\left(\Omega ; \mathbb{R}^{3}\right)$ being complete as a closed subspace of $H^{1}\left(\Omega ; \mathbb{R}^{3}\right)$, there exists $\boldsymbol{v} \in H_{\Gamma_{0}}^{1}\left(\Omega ; \mathbb{R}^{3}\right)$ such that

$$
\boldsymbol{v}_{\sigma(n)} \rightarrow \boldsymbol{v} \text { in } H^{1}\left(\Omega ; \mathbb{R}^{3}\right)
$$

Since its limit satisfies

$$
\mathbf{e}(\boldsymbol{v})=\lim _{n \rightarrow \infty} \mathbf{e}\left(\boldsymbol{v}_{\sigma(n)}\right)=0
$$


it follows that $\boldsymbol{v}=0$ by Part (iii). But this contradicts the relation $\|\boldsymbol{v}\|_{H^{1}\left(\Omega ; \mathbb{R}^{3}\right)}=$ $\lim _{n \rightarrow \infty}\left\|\boldsymbol{v}_{\sigma(n)}\right\|_{H^{1}\left(\Omega ; \mathbb{R}^{3}\right)}=1$, and the proof is complete.

The inequality established in Part (ii) of the proof is called Korn's inequality without boundary conditions.

The uniqueness result established in Part (iii) of the proof is called the infinitesimal rigid displacement lemma. It shows that an infinitesimal rigid displacement field, i.e., a vector field $\boldsymbol{v} \in H^{1}\left(\Omega ; \mathbb{R}^{3}\right)$ satisfying $\mathbf{e}(\boldsymbol{v})=\mathbf{0}$, is necessarily of the form

$$
\boldsymbol{v}(x)=\mathbf{a}+\boldsymbol{b} \wedge \boldsymbol{x} \text { for all } x \in \Omega, \text { where } \mathbf{a}, \boldsymbol{b} \in \mathbb{R}^{3} .
$$

Remark. In the special case where $\Gamma_{0}=\partial \Omega$, Korn's inequality is a trivial consequence of the identity

$$
\int_{\Omega}|\mathbf{e}(\boldsymbol{v})|^{2} d x=\int_{\Omega}|\boldsymbol{\nabla} \boldsymbol{v}|^{2} d x \text { for all } \boldsymbol{v} \in H_{0}^{1}\left(\Omega ; \mathbb{R}^{3}\right),
$$

itself obtained by applying twice the formula of integration by parts (see Section 1.1).

We are now in a position to establish that the equations of linearized threedimensional elasticity have weak solutions. We distinguish two cases depending on whether area $\Gamma_{0}>0$ or not.

Theorem 1.6-2. Assume that the Lamé constants satisfy $\lambda \geq 0$ and $\mu>0$ and that the densities of the applied forces satisfy $\boldsymbol{f} \in L^{6 / 5}\left(\Omega ; \mathbb{R}^{3}\right)$ and $\boldsymbol{g} \in L^{4 / 3}\left(\Gamma_{1} ; \mathbb{R}^{3}\right)$.

If area $\Gamma_{0}>0$, the variational problem (1.6-1) has a unique solution in the space

$$
H_{\Gamma_{0}}^{1}\left(\Omega ; \mathbb{R}^{3}\right):=\left\{\boldsymbol{v} \in H^{1}\left(\Omega ; \mathbb{R}^{3}\right) ; \boldsymbol{v}=\mathbf{0} \text { on } \Gamma_{0}\right\} .
$$

Proof. It suffices to apply the Lax-Milgram lemma to the variational equation (1.6-1), since all its assumptions are clearly satisfied. In particular, the coerciveness of the bilinear form appearing in the left-hand side of the equation (1.6-1) is a consequence of Korn's inequality established in the previous theorem combined with the positiveness of the Lamé constants, which together imply that, for all $\boldsymbol{v} \in H_{\Gamma_{0}}^{1}\left(\Omega ; \mathbb{R}^{3}\right)$,

$$
\begin{aligned}
\int_{\Omega} \boldsymbol{\sigma}: \mathbf{e}(\boldsymbol{v}) d x & =\int_{\Omega}\left(\lambda[\operatorname{tr}(\mathbf{e}(\boldsymbol{v}))]^{2}+2 \mu|\mathbf{e}(\boldsymbol{v})|^{2}\right) d x \\
& \geq 2 \mu \int_{\Omega}|\mathbf{e}(\boldsymbol{v})|^{2} d x \geq C\|\boldsymbol{v}\|_{H^{1}\left(\Omega ; \mathbb{R}^{3}\right)}^{2}
\end{aligned}
$$

Theorem 1.6-3. Assume that the Lamé constants satisfy $\lambda \geq 0$ and $\mu>0$ and that the densities of the applied forces satisfy $\boldsymbol{f} \in L^{6 / 5}\left(\Omega ; \mathbb{R}^{3}\right)$ and $\boldsymbol{g} \in L^{4 / 3}\left(\partial \Omega ; \mathbb{R}^{3}\right)$.

If area $\Gamma_{0}=0$ and $\int_{\Omega} \boldsymbol{f} \cdot \boldsymbol{w} d x+\int_{\partial \Omega} \boldsymbol{g} \cdot \boldsymbol{w} d a=0$ for all $\boldsymbol{w} \in H^{1}\left(\Omega ; \mathbb{R}^{3}\right)$ satisfying $\mathbf{e}(\boldsymbol{w})=\mathbf{0}$, then the variational problem (1.6-1) has a solution in $H^{1}\left(\Omega ; \mathbb{R}^{3}\right)$, unique up to an infinitesimal rigid displacement field.

Sketch of proof. It is again based on the Lax-Milgram lemma applied to the variational equations (1.6-1), this time defined over the quotient space $H^{1}\left(\Omega ; \mathbb{R}^{3}\right) / \boldsymbol{R}_{0}$, 
where $\boldsymbol{R}_{0}$ is the subspace of $H^{1}\left(\Omega ; \mathbb{R}^{3}\right)$ consisting of all the infinitesimal rigid displacements fields. By the infinitesimal rigid displacement lemma (see Part (ii) of the proof of Theorem 1.6-1), $\boldsymbol{R}_{0}$ is the finite-dimensional space

$$
\left\{\boldsymbol{w}: \Omega \rightarrow \mathbb{R}^{3} ; \boldsymbol{w}(x)=\mathbf{a}+\boldsymbol{b} \wedge \boldsymbol{x}, \mathbf{a}, \boldsymbol{b} \in \mathbb{R}^{3}\right\} .
$$

The compatibility relations satisfied by the applied forces imply that the variational equation (1.6-1) is well defined over the quotient space $H^{1}\left(\Omega ; \mathbb{R}^{3}\right) / \boldsymbol{R}_{0}$, which is a Hilbert space with respect to the norm

$$
\|\dot{\boldsymbol{v}}\|_{H^{1}\left(\Omega ; \mathbb{R}^{3}\right) / \boldsymbol{R}_{0}}=\inf _{\boldsymbol{w} \in \boldsymbol{R}_{0}}\|\boldsymbol{v}+\boldsymbol{w}\|_{H^{1}\left(\Omega ; \mathbb{R}^{3}\right)} .
$$

The coerciveness of the bilinear form appearing in the left-hand side of the equation $(1.6-1)$ is then established as a consequence of another Korn's inequality:

$$
\|\mathbf{e}(\dot{\boldsymbol{v}})\|_{L^{2}\left(\Omega ; \mathbb{S}^{3}\right)} \geq C\|\dot{\boldsymbol{v}}\|_{H^{1}\left(\Omega ; \mathbb{R}^{3}\right) / \boldsymbol{R}_{0}} \text { for all } \dot{\boldsymbol{v}} \in H^{1}\left(\Omega ; \mathbb{R}^{3}\right) / \boldsymbol{R}_{0} .
$$

The proof of this inequality follows that of Theorem 1.6-1, with Part (iii) adapted as follows: The sequence $\left(\dot{\boldsymbol{v}}_{n}\right)_{n \in \mathbb{N}}$ is now defined in $H^{1}\left(\Omega ; \mathbb{R}^{3}\right) / \boldsymbol{R}_{0}$ and satisfies

$$
\begin{aligned}
& \left\|\dot{\boldsymbol{v}}_{n}\right\|_{H^{1}\left(\Omega ; \mathbb{R}^{3}\right) / \boldsymbol{R}_{0}}=1 \text { for all } n, \\
& \left\|\mathbf{e}\left(\dot{\boldsymbol{v}}_{n}\right)\right\|_{L^{2}\left(\Omega ; \mathbb{S}^{3}\right)} \rightarrow 0 \text { as } n \rightarrow \infty .
\end{aligned}
$$

Hence there exists an increasing function $\sigma: \mathbb{N} \rightarrow \mathbb{N}$ such that the subsequence $\left(\boldsymbol{v}_{\sigma(n)}\right)$ is a Cauchy sequence in $H^{1}\left(\Omega ; \mathbb{R}^{3}\right)$. This space being complete, there exists $\boldsymbol{v} \in H^{1}\left(\Omega ; \mathbb{R}^{3}\right)$ such that

$$
\boldsymbol{v}_{\sigma(n)} \rightarrow \boldsymbol{v} \text { in } H^{1}\left(\Omega ; \mathbb{R}^{3}\right),
$$

and its limit satisfies

$$
\mathbf{e}(\boldsymbol{v})=\lim _{n \rightarrow \infty} \mathbf{e}\left(\boldsymbol{v}_{\sigma(n)}\right)=\mathbf{0} .
$$

Therefore $\boldsymbol{v} \in \boldsymbol{R}_{0}$ by Part (iii), hence $\left(\boldsymbol{v}_{\sigma(n)}-\boldsymbol{v}\right) \rightarrow \mathbf{0}$ in $H^{1}\left(\Omega ; \mathbb{R}^{3}\right)$. This implies that

$$
\left\|\dot{\boldsymbol{v}}_{\sigma(n)}\right\|_{H^{1}\left(\Omega ; \mathbb{R}^{3}\right) / \boldsymbol{R}_{0}} \leq\left\|\boldsymbol{v}_{\sigma(n)}-\boldsymbol{v}\right\|_{H^{1}\left(\Omega ; \mathbb{R}^{3}\right)} \rightarrow \mathbf{0} \text { as } n \rightarrow \infty,
$$

which contradicts the relation $\left\|\dot{\boldsymbol{v}}_{\sigma(n)}\right\|_{H^{1}\left(\Omega ; \mathbb{R}^{3}\right) / \boldsymbol{R}_{0}}=1$ for all $n$.

The variational problem (1.6-1) is called a pure displacement problem when $\Gamma_{0}=\partial \Omega$, a pure traction problem when $\Gamma_{1}=\partial \Omega$, and a displacementtraction problem when area $\Gamma_{0}>0$ and area $\Gamma_{1}>0$.

Since the system of partial differential equations associated with the linear threedimensional variational model is elliptic, we expect the solution of the latter to be regular if the data $\boldsymbol{f}, \boldsymbol{g}$, and $\partial \Omega$ are regular and if there is no change of boundary condition along a connected portion of $\partial \Omega$. More specifically, the following regularity results hold (indications about the proof are given in Ciarlet [18, Theorem $6.3-6])$.

Theorem 1.6-4 (pure displacement problem). Assume that $\Gamma_{0}=\partial \Omega$. If $\boldsymbol{f} \in W^{m, p}\left(\Omega ; \mathbb{R}^{3}\right)$ and $\partial \Omega$ is of class $\mathcal{C}^{m+2}$ for some integer $m \geq 0$ and real number $1<p<\infty$ satisfying $p \geq \frac{6}{5+2 m}$, then the solution $\boldsymbol{u}$ to the variational equation (1.6-1) is in the space $W^{m+2, p}\left(\Omega ; \mathbb{R}^{3}\right)$ and there exists a constant $C$ such that

$$
\|\boldsymbol{u}\|_{W^{m+2, p}\left(\Omega ; \mathbb{R}^{3}\right)} \leq C\|\boldsymbol{f}\|_{W^{m+2, p}\left(\Omega ; \mathbb{R}^{3}\right)} .
$$


Furthermore, $\boldsymbol{u}$ satisfies the boundary value problem:

$$
\begin{aligned}
-\operatorname{div} \boldsymbol{\sigma}(x) & =\boldsymbol{f}, & & x \in \Omega, \\
\boldsymbol{u}(x) & =\mathbf{0}, & & x \in \partial \Omega .
\end{aligned}
$$

Theorem 1.6-5 (pure traction problem). Assume that $\Gamma_{1}=\partial \Omega$ and $\int_{\Omega} \boldsymbol{f}$. $\boldsymbol{w} d x+\int_{\partial \Omega} \boldsymbol{g} \cdot \boldsymbol{w} d a=0$ for all vector fields $\boldsymbol{v} \in H^{1}\left(\Omega ; \mathbb{R}^{3}\right)$ satisfying $\mathbf{e}(\boldsymbol{w})=0$. If $\boldsymbol{f} \in W^{m, p}\left(\Omega ; \mathbb{R}^{3}\right), \boldsymbol{g} \in W^{m+1-1 / p, p}\left(\Gamma_{1} ; \mathbb{R}^{3}\right)$, and $\partial \Omega$ is of class $\mathcal{C}^{m+2}$ for some integer $m \geq 0$ and real number $1<p<\infty$ satisfying $p \geq \frac{6}{5+2 m}$, then any solution $\boldsymbol{u}$ to the variational equation $(1.6-1)$ is in the space $W^{m+2, p}\left(\Omega ; \mathbb{R}^{3}\right)$ and there exist a constant $C$ such that

$$
\|\dot{\boldsymbol{u}}\|_{W^{m+2, p}\left(\Omega ; \mathbb{R}^{3}\right) / \boldsymbol{R}_{0}} \leq C\left(\|\boldsymbol{f}\|_{W^{m+2, p}\left(\Omega ; \mathbb{R}^{3}\right)}+\|\boldsymbol{g}\|_{W^{m+1-1 / p, p}\left(\partial \Omega ; \mathbb{R}^{3}\right)}\right) .
$$

Furthermore, $\boldsymbol{u}$ satisfies the boundary value problem:

$$
\begin{aligned}
-\operatorname{div} \boldsymbol{\sigma}(x) & =\boldsymbol{f}(x), & & x \in \Omega, \\
\boldsymbol{\sigma}(x) \boldsymbol{n}(x) & =\boldsymbol{g}(x), & & x \in \partial \Omega .
\end{aligned}
$$

\subsection{EXISTENCE THEORY IN NONLINEAR THREE-DIMENSIONAL ELASTICITY BY THE IMPLICIT FUNCTION THEOREM}

The question of whether the equations of nonlinear three-dimensional elasticity have solutions has been answered in the affirmative when the data satisfy some specific assumptions, but remains open in the other cases. To this day, there are two theories of existence, one based on the implicit function theorem, and one, due to John Ball, based on the minimization of functionals.

We state here the existence theorems provided by both theories but we will provide the proof only for the existence theorem based on the implicit function theorem. For the existence theorem based on the minimization of functionals we will only sketch of the proof of John Ball (Section 1.8).

The existence theory based on the implicit function theorem asserts that the equations of nonlinear three-dimensional elasticity have solutions if the solutions to the associated equations of linearized three-dimensional elasticity are smooth enough, and the applied forces are small enough. The first requirement essentially means that the bodies are either held fixed along their entire boundary (i.e., $\Gamma_{0}=$ $\partial \Omega$ ), or nowhere along their boundary (i.e., $\Gamma_{1}=\partial \Omega$ ).

We restrict our presentation to the case of elastic bodies made of a St VenantKirchhoff material. In other words, we assume throughout this section that

$$
\boldsymbol{\Sigma}=\lambda(\operatorname{tr} \boldsymbol{E}) \boldsymbol{I}+2 \mu \boldsymbol{E} \text { and } \boldsymbol{E}=\frac{1}{2}\left(\boldsymbol{\nabla} \boldsymbol{u}^{T}+\boldsymbol{\nabla} \boldsymbol{u}+\boldsymbol{\nabla} \boldsymbol{u}^{T} \boldsymbol{\nabla} \boldsymbol{u}\right),
$$

where $\lambda>0$ and $\mu>0$ are the Lamé constants of the material and $\boldsymbol{u}: \Omega \rightarrow \mathbb{R}^{3}$ is the unknown displacement field. We assume that $\Gamma_{0}=\partial \Omega$ (the case where $\Gamma_{1}=$ $\partial \Omega$ requires some extra care because the space of infinitesimal rigid displacements fields does not reduce to $\{\mathbf{0}\}$ ). Hence the equations of nonlinear three-dimensional elasticity assert that the displacement field $\boldsymbol{u}: \Omega \rightarrow \mathbb{R}^{3}$ inside the body is the solution to the boundary value problem

$$
\begin{aligned}
-\operatorname{div}((\boldsymbol{I}+\boldsymbol{\nabla} \boldsymbol{u}) \boldsymbol{\Sigma}) & =\boldsymbol{f} \text { in } \Omega, \\
\boldsymbol{u} & =\mathbf{0} \text { on } \partial \Omega,
\end{aligned}
$$


where the field $\boldsymbol{\Sigma}$ is given in terms of the unknown field $\boldsymbol{u}$ by means of (1.7-1). The existence result is then the following

Theorem 1.7-1. The nonlinear boundary value problem (1.7-1)-(1.7-2) has a solution $\boldsymbol{u} \in W^{2, p}\left(\Omega ; \mathbb{R}^{3}\right)$ if $\Omega$ is a domain with a boundary $\partial \Omega$ of class $\mathcal{C}^{2}$, and for some $p>3, \boldsymbol{f} \in L^{p}\left(\Omega ; \mathbb{R}^{3}\right)$ and $\|\boldsymbol{f}\|_{L^{p}\left(\Omega ; \mathbb{R}^{3}\right)}$ is small enough.

Proof. Define the spaces

$$
\begin{aligned}
& \boldsymbol{X}:=\left\{\boldsymbol{v} \in W^{2, p}\left(\Omega ; \mathbb{R}^{3}\right) ; \boldsymbol{v}=\mathbf{0} \text { on } \partial \Omega\right\}, \\
& \boldsymbol{Y}:=L^{p}\left(\Omega ; \mathbb{R}^{3}\right) .
\end{aligned}
$$

Define the nonlinear mapping $\mathcal{F}: \boldsymbol{X} \rightarrow \boldsymbol{Y}$ by

$$
\mathcal{F}(\boldsymbol{v}):=-\operatorname{div}((\boldsymbol{I}+\boldsymbol{\nabla} \boldsymbol{v}) \boldsymbol{\Sigma}) \text { for any } \boldsymbol{v} \in \boldsymbol{X},
$$

where

$$
\boldsymbol{\Sigma}=\lambda(\operatorname{tr} \boldsymbol{E}) \boldsymbol{I}+2 \mu \boldsymbol{E} \text { and } \boldsymbol{E}=\frac{1}{2}\left(\boldsymbol{\nabla} \boldsymbol{v}^{T}+\boldsymbol{\nabla} \boldsymbol{v}+\boldsymbol{\nabla} \boldsymbol{v}^{T} \boldsymbol{\nabla} \boldsymbol{v}\right)
$$

It suffices to prove that the equation

$$
\mathcal{F}(\boldsymbol{u})=\boldsymbol{f}
$$

has solutions in $\boldsymbol{X}$ provided that the norm of $\boldsymbol{f}$ in the space $\boldsymbol{Y}$ is small enough.

The idea for solving the above equation is as follows. If the norm of $\boldsymbol{f}$ is small, we expect the norm of $\boldsymbol{u}$ to be small too, so that the above equation can be written as

$$
\mathcal{F}(\mathbf{0})+\mathcal{F}^{\prime}(\mathbf{0}) \boldsymbol{u}+o\left(\|\boldsymbol{u}\|_{\boldsymbol{X}}\right)=\boldsymbol{f},
$$

Since $\mathcal{F}(\mathbf{0})=\mathbf{0}$, we expect the above equation to have solution if the linear equation

$$
\mathcal{F}^{\prime}(\mathbf{0}) \boldsymbol{u}=\boldsymbol{f}
$$

has solutions in $\boldsymbol{X}$. But this equation is exactly the system of equations of linearized three-dimensional elasticity. Hence, as we shall see, this equation has solutions in $\boldsymbol{X}$ thanks to Theorem 1.6-4.

In order to solve the nonlinear equation $\mathcal{F}(\boldsymbol{u})=\boldsymbol{f}$, it is thus natural to apply the inverse function theorem (see, e.g., Taylor [85]). According to this theorem, if $\mathcal{F}$ : $\boldsymbol{X} \rightarrow \boldsymbol{Y}$ is of class $\mathcal{C}^{1}$ and the Fréchet derivative $\mathcal{F}^{\prime}(\mathbf{0}): \boldsymbol{X} \rightarrow \boldsymbol{Y}$ is an isomorphism (i.e., an operator that is linear, bijective, and continuous with a continuous inverse), then there exist two open sets $U \subset \boldsymbol{X}$ and $V \subset \boldsymbol{Y}$ with $\mathbf{0} \in U$ and $\mathbf{0}=\mathcal{F}(\mathbf{0}) \in V$ such that, for all $\boldsymbol{f} \in V$, there exists a unique element $\boldsymbol{u} \in U$ satisfying the equation

$$
\mathcal{F}(\boldsymbol{u})=\boldsymbol{f}
$$

Furthermore, the mapping

$$
\boldsymbol{f} \in V \mapsto \boldsymbol{u} \in U
$$

is of class $\mathcal{C}^{1}$.

It remains to prove that the assumptions of the inverse function theorem are indeed satisfied. First, the function $\mathcal{F}$ is well defined (i.e., $\mathcal{F}(\boldsymbol{u}) \in \boldsymbol{Y}$ for all $\boldsymbol{u} \in \boldsymbol{X}$ ) since the space $W^{1, p}(\Omega)$ is an algebra (thanks to the assumption $p>3$ ). Second, the function $\mathcal{F}: \boldsymbol{X} \rightarrow \boldsymbol{Y}$ is of class $\mathcal{C}^{1}$ since it is multilinear (in fact, $\mathcal{F}$ is even of class $\left.\mathcal{C}^{\infty}\right)$. Third, the Fréchet derivative of $\mathcal{F}$ is given by

$$
\mathcal{F}^{\prime}(0) u=-\operatorname{div} \sigma,
$$

where

$$
\boldsymbol{\sigma}:=\lambda(\operatorname{tr} \mathbf{e}) \boldsymbol{I}+2 \mu \mathbf{e} \text { and } \mathbf{e}:=\frac{1}{2}\left(\boldsymbol{\nabla} \boldsymbol{u}^{T}+\boldsymbol{\nabla} \boldsymbol{u}\right),
$$


from which we infer that the equation $\mathcal{F}^{\prime}(\mathbf{0}) \boldsymbol{u}=\boldsymbol{f}$ is exactly the equations of linearized three-dimensional elasticity (see (1.4-3)-(1.4-4) with $\Gamma_{0}=\partial \Omega$ ). Therefore, Theorem 1.6-4 shows that the function $\mathcal{F}^{\prime}(\mathbf{0}): \boldsymbol{X} \rightarrow \boldsymbol{Y}$ is an isomorphism

Since all the hypotheses of the inverse function theorem are satisfied, the equations of nonlinear three-dimensional elasticity (1.7-1)-(1.7-2) have a unique solution in the neighborhood $U$ of the origin in $W^{2, p}\left(\Omega ; \mathbb{R}^{3}\right)$ if $\boldsymbol{f}$ belongs to the neighborhood $V$ of the origin in $L^{p}\left(\Omega ; \mathbb{R}^{3}\right)$. In particular, if $\delta>0$ is the radius of a ball $B(\mathbf{0}, \delta)$ contained in $V$, then the problem (1.7-1)-(1.7-2) has solutions for all $\|\boldsymbol{f}\|_{L^{p}(\Omega)}<\delta$.

The unique solution $\boldsymbol{u}$ in the neighborhood $U$ of the origin in $W^{2, p}\left(\Omega ; \mathbb{R}^{3}\right)$ of the equations of nonlinear three-dimensional elasticity (1.7-1)-(1.7-2) depends continuously on $\boldsymbol{f}$, i.e., with self-explanatory notation

$$
\boldsymbol{f}_{n} \rightarrow \boldsymbol{f} \text { in } L^{p}\left(\Omega ; \mathbb{R}^{3}\right) \Rightarrow \boldsymbol{u}_{n} \rightarrow \boldsymbol{u} \text { in } W^{2, p}\left(\Omega ; \mathbb{R}^{3}\right) .
$$

This shows that, under the assumptions of Theorem 1.7-1, the system of equations of nonlinear three-dimensional elasticity is well-posed.

Existence results such as Theorem 1.7-1 can be found in Valent [86], Marsden \& Hughes [68], Ciarlet \& Destuynder [25], who simultaneously and independently established the existence of solutions to the equations of nonlinear three-dimensional elasticity via the implicit function theorem.

\subsection{EXISTENCE THEORY IN NONLINEAR THREE-DIMENSIONAL ELASTICITY BY THE MINIMIZATION OF ENERGY (JOHN BALL'S APPROACH)}

We begin with the definition of hyperelastic materials. Recall that (see Section 1.3) an elastic material has a constitutive equation of the form

$$
\boldsymbol{T}(x):=\boldsymbol{T}^{\sharp}(x, \boldsymbol{\nabla} \boldsymbol{\Phi}(x)) \text { for all } x \in \bar{\Omega},
$$

where $\boldsymbol{T}^{\sharp}: \bar{\Omega} \times \mathbb{M}_{+}^{3} \rightarrow \mathbb{M}^{3}$ is the response function of the material and $\boldsymbol{T}(x)$ is the first Piola-Kirchhoff stress tensor at $x$.

Then an elastic material is hyperelastic if there exists a function $W: \bar{\Omega} \times \mathbb{M}_{+}^{3} \rightarrow$ $\mathbb{R}$, called the stored energy function, such that its response function $\boldsymbol{T}^{\sharp}$ can be fully reconstructed from $W$ by means of the relation

$$
\boldsymbol{T}^{\sharp}(x, \boldsymbol{F})=\frac{\partial W}{\partial \boldsymbol{F}}(x, \boldsymbol{F}) \text { for all }(x, \boldsymbol{F}) \in \bar{\Omega} \times \mathbb{M}_{+}^{3},
$$

where $\frac{\partial W}{\partial \boldsymbol{F}}$ denotes the Fréchet derivative of $W$ with respect to the variable $\boldsymbol{F}$. In other words, at each $x \in \bar{\Omega}, \frac{\partial W}{\partial \boldsymbol{F}}(x, \boldsymbol{F})$ is the unique matrix in $\mathbb{M}^{3}$ that satisfies

$$
W(x, \boldsymbol{F}+\boldsymbol{H})=W(x, \boldsymbol{F})+\frac{\partial W}{\partial \boldsymbol{F}}(x, \boldsymbol{F}): \boldsymbol{H}+o_{x}(|\boldsymbol{H}|)
$$

for all $\boldsymbol{F} \in \mathbb{M}_{+}^{3}$ and $\boldsymbol{H} \in \mathbb{M}^{3}$ (a detailed study of hyperelastic materials can be found in, e.g., Ciarlet [18, Chap. 4]).

John Ball [9] has shown that the minimization problem formally associated with the equations of nonlinear three-dimensional elasticity (see (1.4-1)) when the material constituting the body is hyperelastic has solutions if the function $W$ satisfies certain physically realistic conditions of polyconvexity, coerciveness, and growth. A typical example of such a function $W$, which is called the stored energy function of the material, is given by

$$
W(x, \boldsymbol{F})=a\|\boldsymbol{F}\|^{p}+b\|\operatorname{Cof} \boldsymbol{F}\|^{q}+c|\operatorname{det} \boldsymbol{F}|^{r}-d \log (\operatorname{det} \boldsymbol{F})
$$


for all $\boldsymbol{F} \in \mathbb{M}_{+}^{3}$, where $p \geq 2, q \geq \frac{p}{p-1}, r>1, a>0,, b>0, c>0, d>0$, and $\|\cdot\|$ is the norm defined by $\|\boldsymbol{F}\|:=\left\{\operatorname{tr}\left(\boldsymbol{F}^{T} \boldsymbol{F}\right)\right\}^{1 / 2}$ for all $\boldsymbol{F} \in \mathbb{M}^{3}$.

The major interest of hyperelastic materials is that, for such materials, the equations of nonlinear three-dimensional elasticity are, at least formally, the Euler equation associated with a minimization problem (this property only holds formally because, in general, the solution to the minimization problem does not have the regularity needed to properly establish the Euler equation associated with the minimization problem). To see this, consider first the equations of nonlinear threedimensional elasticity (see Section 1.4):

$$
\begin{aligned}
-\operatorname{div} \boldsymbol{T}^{\sharp}(x, \boldsymbol{\nabla} \boldsymbol{\Phi}(x)) & =\boldsymbol{f}(x), x \in \Omega, \\
\boldsymbol{\Phi}(x) & =x, x \in \Gamma_{0}, \\
\boldsymbol{T}^{\sharp}(x, \boldsymbol{\nabla} \boldsymbol{\Phi}(x)) \boldsymbol{n}(x) & =\boldsymbol{g}(x), x \in \Gamma_{1},
\end{aligned}
$$

where, for simplicity, we have assumed that the applied forces do not depend on the unknown deformation $\boldsymbol{\Phi}$.

A weak solution $\boldsymbol{\Phi}$ to the boundary value problem (1.8-1) is then the solution to the following variational problem, also known as the principle of virtual works:

$$
\int_{\Omega} \boldsymbol{T}^{\sharp}(\cdot, \nabla \boldsymbol{\Phi}): \boldsymbol{\nabla} \boldsymbol{v} d x=\int_{\Omega} \boldsymbol{f} \cdot \boldsymbol{v} d x+\int_{\Gamma_{1}} \boldsymbol{g} \cdot \boldsymbol{v} d a
$$

for all smooth enough vector fields $\boldsymbol{v}: \bar{\Omega} \rightarrow \mathbb{R}^{3}$ such that $\boldsymbol{v}=\mathbf{0}$ on $\Gamma_{0}$.

If the material is hyperelastic, then $\boldsymbol{T}^{\sharp}(x, \boldsymbol{\nabla} \boldsymbol{\Phi}(x))=\frac{\partial W}{\partial \boldsymbol{F}}(x, \boldsymbol{\nabla} \boldsymbol{\Phi}(x))$, and the above equation can be written as

$$
J^{\prime}(\boldsymbol{\Phi}) \boldsymbol{v}=0,
$$

where $J^{\prime}$ is the Fréchet derivative of the functional $J$ defined by

$$
J(\Psi):=\int_{\Omega} W(x, \nabla \boldsymbol{\Psi}(x)) d x-\int_{\Omega} \boldsymbol{f} \cdot \mathbf{\Psi} d x-\int_{\Gamma_{1}} \boldsymbol{g} \cdot \boldsymbol{\Psi} d a,
$$

for all smooth enough vector fields $\Psi: \bar{\Omega} \rightarrow \mathbb{R}^{3}$ such that $\boldsymbol{\Psi}=$ id on $\Gamma_{0}$. The functional $J$ is called the total energy.

Therefore the variational equations associated with the equations of nonlinear three-dimensional elasticity are, at least formally, the Euler equations associated with the minimization problem

$$
J(\mathbf{\Phi})=\min _{\mathbf{\Psi} \in \mathcal{M}} J(\mathbf{\Psi}),
$$

where $\mathcal{M}$ is an appropriate set of all admissible deformations $\Psi: \Omega \rightarrow \mathbb{R}^{3}$ (an example is given in the next theorem).

John Ball's theory provides an existence theorem for this minimization problem when the function $W$ satisfies the following fundamental definition (see [9]): A stored energy function $W: \bar{\Omega} \times \mathbb{M}_{+}^{3} \rightarrow \mathbb{R}$ is said to be polyconvex if, for each $x \in \bar{\Omega}$, there exists a convex function $\mathcal{W}(x, \cdot): \mathbb{M}^{3} \times \mathbb{M}^{3} \times(0, \infty) \rightarrow \mathbb{R}$ such that

$$
W(x, \boldsymbol{F})=\mathcal{W}(x, \boldsymbol{F}, \operatorname{Cof} \boldsymbol{F}, \operatorname{det} \boldsymbol{F}) \text { for all } \boldsymbol{F} \in \mathbb{M}_{+}^{3} \text {. }
$$

Theorem 1.8-1 (John Ball). Let $\Omega$ be a domain in $\mathbb{R}^{3}$ and let $W$ be a polyconvex function that satisfies the following properties:

The function $\mathcal{W}(\cdot, \boldsymbol{F}, \boldsymbol{H}, \delta): \Omega \rightarrow \mathbb{R}$ is measurable for all $(\boldsymbol{F}, \boldsymbol{H}, \delta) \in \mathbb{M}^{3} \times \mathbb{M}^{3} \times$ $(0, \infty)$. 
There exist numbers $p \geq 2, q \geq \frac{p}{p-1}, r>1, \alpha>0$, and $\beta \in \mathbb{R}$ such that

$$
W(x, \boldsymbol{F}) \geq \alpha\left(\|\boldsymbol{F}\|^{p}+\|\mathbf{C o f} \boldsymbol{F}\|^{q}+|\operatorname{det} \boldsymbol{F}|^{r}\right)-\beta
$$

for almost all $x \in \Omega$ and for all $\boldsymbol{F} \in \mathbb{M}_{+}^{3}$.

For almost all $x \in \Omega, W(x, \boldsymbol{F}) \rightarrow+\infty$ if $\boldsymbol{F} \in \mathbb{M}_{+}^{3}$ is such that $\operatorname{det} \boldsymbol{F} \rightarrow 0$.

Let $\Gamma_{1}$ be a relatively open subset of $\partial \Omega$, let $\Gamma_{0}:=\partial \Omega \backslash \Gamma_{1}$, and let there be given fields $\boldsymbol{f} \in L^{6 / 5}\left(\Omega ; \mathbb{R}^{3}\right)$ and $\boldsymbol{g} \in L^{4 / 3}\left(\Gamma_{1} ; \mathbb{R}^{3}\right)$. Define the functional

$$
J(\boldsymbol{\Psi}):=\int_{\Omega} W(x, \boldsymbol{\nabla} \mathbf{\Psi}(x)) d x-\int_{\Omega} \boldsymbol{f}(x) \cdot \mathbf{\Psi}(x) d x-\int_{\Gamma_{1}} \boldsymbol{g}(x) \cdot \mathbf{\Psi}(x) d a,
$$

and the set

$$
\begin{aligned}
\mathcal{M}:=\left\{\boldsymbol{\Psi} \in W^{1, p}\left(\Omega ; \mathbb{R}^{3}\right) ;\right. & \operatorname{Cof}(\boldsymbol{\nabla} \Psi) \in L^{q}\left(\Omega ; \mathbb{M}^{3}\right), \operatorname{det}(\boldsymbol{\nabla} \boldsymbol{\Psi}) \in L^{r}((\Omega), \\
& \left.\operatorname{det}(\boldsymbol{\nabla} \boldsymbol{\Psi})>0 \text { a.e. in } \Omega, \boldsymbol{\Psi}=\mathbf{i d} \text { on } \Gamma_{0}\right\} .
\end{aligned}
$$

Finally, assume that area $\Gamma_{0}>0$ and that $\inf _{\boldsymbol{\Psi} \in \mathcal{M}} J(\boldsymbol{\Psi})<\infty$.

Then there exists $\boldsymbol{\Phi} \in \mathcal{M}$ such that

$$
J(\mathbf{\Phi})=\inf _{\boldsymbol{\Psi} \in \mathcal{M}} J(\boldsymbol{\Psi}) .
$$

Sketch of proof (see Ball [9], or Ciarlet [18], for a detailed proof). Let $\boldsymbol{\Phi}_{n}$ be a infimizing sequence of the functional $J$, i.e., a sequence of vector fields $\boldsymbol{\Phi}_{n} \in \mathcal{M}$ such that

$$
J\left(\mathbf{\Phi}_{n}\right) \rightarrow \inf _{\boldsymbol{\Psi} \in \mathcal{M}} J(\boldsymbol{\Psi})<\infty .
$$

The coerciveness assumption on $W$ implies that the sequences $\left(\boldsymbol{\Phi}_{n}\right),\left(\mathbf{C o f}\left(\boldsymbol{\nabla} \boldsymbol{\Phi}_{n}\right)\right)$, and $\left(\operatorname{det}\left(\boldsymbol{\nabla} \boldsymbol{\Phi}_{n}\right)\right)$ are bounded respectively in the spaces $W^{1, p}\left(\Omega ; \mathbb{R}^{3}\right), L^{q}\left(\Omega ; \mathbb{M}^{3}\right)$, and $L^{r}(\Omega)$. Since these spaces are reflexive, there exist subsequences $\left(\boldsymbol{\Phi}_{\sigma(n)}\right)$, $\left(\operatorname{Cof}\left(\boldsymbol{\nabla} \boldsymbol{\Phi}_{\sigma(n)}\right)\right)$, and $\left(\operatorname{det}\left(\boldsymbol{\nabla} \boldsymbol{\Phi}_{\sigma(n)}\right)\right)$ such that ( $\rightarrow$ denotes weak convergence)

$$
\begin{aligned}
\boldsymbol{\Phi}_{\sigma(n)} \rightarrow \boldsymbol{\Phi} & \text { in } W^{1, p}\left(\Omega ; \mathbb{R}^{3}\right), \\
\boldsymbol{H}_{\sigma(n)}:=\operatorname{Cof}\left(\boldsymbol{\nabla} \boldsymbol{\Phi}_{\sigma(n)}\right) \rightarrow \boldsymbol{H} & \text { in } L^{q}\left(\Omega ; \mathbb{M}^{3}\right), \\
\delta_{\sigma(n)}:=\operatorname{det}\left(\boldsymbol{\nabla} \boldsymbol{\Phi}_{\sigma(n)}\right) \rightarrow \delta & \text { in } L^{r}(\Omega) .
\end{aligned}
$$

For all $\boldsymbol{\Phi} \in W^{1, p}\left(\Omega ; \mathbb{R}^{3}\right), \boldsymbol{H} \in L^{q}\left(\Omega ; \mathbb{M}^{3}\right)$, and $\delta \in L^{r}(\Omega)$ with $\delta>0$ almost everywhere in $\Omega$, define the functional

$$
\begin{aligned}
\mathcal{J}(\boldsymbol{\Phi}, \boldsymbol{H}, \delta):=\int_{\Omega} \mathcal{W}(x, \boldsymbol{\nabla} \boldsymbol{\Phi}(x), \boldsymbol{H}(x), \delta(x)) d x & \\
& -\int_{\Omega} \boldsymbol{f}(x) \cdot \mathbf{\Phi}(x) d x-\int_{\Gamma_{1}} \boldsymbol{g}(x) \cdot \mathbf{\Phi}(x) d a,
\end{aligned}
$$

where, for each $x \in \Omega, \mathcal{W}(x, \cdot): \mathbb{M}^{3} \times \mathbb{M}^{3} \times(0, \infty) \rightarrow \mathbb{R}$ is the function given by the polyconvexity assumption on $W$. Since $\mathcal{W}(x, \cdot)$ is convex, the above weak convergences imply that

$$
\mathcal{J}(\boldsymbol{\Phi}, \boldsymbol{H}, \delta) \leq \liminf _{n \rightarrow \infty} \mathcal{J}\left(\boldsymbol{\Phi}_{\sigma(n)}, \boldsymbol{H}_{\sigma(n)}, \delta_{\sigma(n)}\right) .
$$

But $\mathcal{J}\left(\boldsymbol{\Phi}_{\sigma(n)}, \boldsymbol{H}_{\sigma(n)}, \delta_{\sigma(n)}\right)=J\left(\boldsymbol{\Phi}_{\sigma(n)}\right)$ and $J\left(\boldsymbol{\Phi}_{n}\right) \rightarrow \inf _{\boldsymbol{\Psi} \in \mathcal{M}} J(\boldsymbol{\Psi})$. Therefore $\mathcal{J}(\boldsymbol{\Phi}, \boldsymbol{H}, \delta)=\inf _{\boldsymbol{\Psi} \in \mathcal{M}} J(\boldsymbol{\Psi})$.

A compactness by compensation argument applied to the weak convergences above then shows that

$$
\boldsymbol{H}=\boldsymbol{C o f}(\boldsymbol{\nabla} \boldsymbol{\Phi}) \text { and } \delta=\operatorname{det}(\boldsymbol{\nabla} \boldsymbol{\Phi}) .
$$


Hence $J(\boldsymbol{\Phi})=\mathcal{J}(\boldsymbol{\Phi}, \boldsymbol{H}, \delta)$.

It remains to prove that $\boldsymbol{\Phi} \in \mathcal{M}$. The property that $W(\boldsymbol{F}) \rightarrow+\infty$ if $\boldsymbol{F} \in \mathbb{M}_{+}$ is such that $\operatorname{det} \boldsymbol{F} \rightarrow 0$, then implies that $\operatorname{det}(\boldsymbol{\nabla} \boldsymbol{\Phi})>0$ a.e. in $\Omega$. Finally, since $\boldsymbol{\Phi}_{n} \rightarrow \boldsymbol{\Phi}$ in $W^{1, p}\left(\Omega ; \mathbb{R}^{3}\right)$ and since the trace operator is linear, it follows that $\boldsymbol{\Phi}=$ id on $\Gamma_{0}$. Hence $\boldsymbol{\Phi} \in \mathcal{M}$.

Since $J(\boldsymbol{\Phi})=\mathcal{J}(\boldsymbol{\Phi}, \boldsymbol{H}, \delta)=\inf _{\boldsymbol{\Psi} \in \mathcal{M}} J(\boldsymbol{\Psi})$, the weak limit $\boldsymbol{\Phi}$ of the sequence $\boldsymbol{\Phi}_{\sigma(n)}$ satisfies the conditions of the theorem.

A St Venant-Kirchhoff material with Lamé constants $\lambda>0$ and $\mu>0$ is hyperelastic, but not polyconvex. However, Ciarlet \& Geymonat [26] have shown that the stored energy function of a St Venant-Kirchhoff material, which is given by

$$
W(\boldsymbol{F})=\frac{\lambda}{8}\left(\operatorname{tr}\left(\boldsymbol{F}^{T} \boldsymbol{F}-\boldsymbol{I}\right)\right)^{2}+\frac{\mu}{4}\left\|\boldsymbol{F}^{T} \boldsymbol{F}-\boldsymbol{I}\right\|^{2},
$$

can be "approximated" with polyconvex stored energy functions in the following sense: There exists polyconvex stored energy functions of the form

$$
W^{b}(\boldsymbol{F})=a\|\boldsymbol{F}\|^{2}+b\|\mathbf{C o f} \boldsymbol{F}\|^{2}+c|\operatorname{det} \boldsymbol{F}|^{2}-d \log (\operatorname{det} \boldsymbol{F})+e
$$

with $a>0, b>0, c>0, d>0, e \in \mathbb{R}$, that satisfy

$$
W^{b}(\boldsymbol{F})=W(\boldsymbol{F})+\mathcal{O}\left(\left\|\boldsymbol{F}^{T} \boldsymbol{F}-\boldsymbol{I}\right\|^{3}\right) .
$$

A stored energy function of this form possesses all the properties required for applying Theorem 1.8-1. In particular, it satisfies the coerciveness inequality:

$$
W^{b}(\boldsymbol{F}) \geq \alpha\left(\|\boldsymbol{F}\|^{2}+\|\operatorname{Cof} \boldsymbol{F}\|^{2}+(\operatorname{det} \boldsymbol{F})^{2}\right)+\beta \text {, with } \alpha>0 \text { and } \beta \in \mathbb{R} \text {. }
$$




\section{Part 2. TWO-DIMENSIONAL THEORY}

\section{OUtLine}

In the first part of the article, we have seen how an elastic body subjected to applied forces and appropriate boundary conditions can be modeled by the equations of nonlinear or linearized three-dimensional elasticity. Clearly, these equations can be used in particular to model an elastic shell, which is nothing but an elastic body whose reference configuration has a particular shape.

In the second part of the article, we will show how an elastic shell can be modeled by equations defined on a two-dimensional domain. These new equations may be viewed as a simplification of the equations of three-dimensional elasticity, obtained by eliminating some of the terms of lesser order of magnitude with respect to the thickness of the shell. This simplification is done by exploiting the special geometry of the reference configuration of the shell, and especially, the assumed "smallness" of the thickness of the shell.

In the next section, we begin our study with a brief review of the geometry of surfaces in $\mathbb{R}^{3}$ defined by curvilinear coordinates. Of special importance are their first and second fundamental forms.

In Section 2.2, we define the reference configuration of a shell as the set in $\mathbb{R}^{3}$ formed by all points within a distance $\leq \varepsilon$ from a given surface in $\mathbb{R}^{3}$. This surface is the middle surface of the shell and $\varepsilon$ is its half-thichness. We then define a system of three-dimensional "natural" curvilinear coordinates inside the reference configuration of a shell.

In Section 2.3, the equations of nonlinear or linearized three-dimensional elasticity, which were written in Cartesian coordinates in the first part of the article, are recast in terms of these natural curvilinear coordinates, as a preliminary step toward the derivation of two-dimensional shell theories.

In Section 2.5, we give a brief account of the derivation of nonlinear membrane and flexural shell models by letting the thickness $\varepsilon$ approach zero in the equations of nonlinear three-dimensional elasticity in curvilinear coordinates. The same program is applied in Section 2.6 to the equations of linearized three-dimensional elasticity in curvilinear coordinates to derive the linearized membrane and flexural shell models.

In Sections 2.7-2.10, we study the nonlinear and linear Koiter shell models. The energy of the nonlinear Koiter shell model is defined in terms of the covariant components of the change of metric and change of curvature tensor fields associated with a displacement field of the middle surface of the reference configuration of the shell. The linear Koiter shell model is then defined by linearizing the above tensor fields. Finally, the existence and uniqueness of solutions to the linear Koiter shell equations are established, thanks to a fundamental Korn inequality on a surface and to an infinitesimal rigid displacement lemma on a surface.

\subsection{A Quick Review of the Differential geometry of SURfaces in $\mathbb{R}^{3}$}

To begin with, we briefly recapitulate some important notions of differential geometry of surfaces (for detailed expositions, see, e.g., Ciarlet $[22,23]$ ).

Greek indices and exponents (except $\nu$ in the notation $\partial_{\nu}$ ) range in the set $\{1,2\}$, Latin indices and exponents range in the set $\{1,2,3\}$ (save when they are used for indexing sequences), and the summation convention with respect to repeated indices and exponents is systematically used. 
Let $\omega$ be a domain in $\mathbb{R}^{2}$. Let $y=\left(y_{\alpha}\right)$ denote a generic point in the set $\bar{\omega}$ and let $\partial_{\alpha}:=\partial / \partial y_{\alpha}$. Let there be given an immersion $\boldsymbol{\theta} \in \mathcal{C}^{3}\left(\bar{\omega} ; \mathbb{R}^{3}\right)$, i.e., a mapping such that the two vectors

$$
\mathbf{a}_{\alpha}(y):=\partial_{\alpha} \boldsymbol{\theta}(y)
$$

are linearly independent at all points $y \in \bar{\omega}$. These two vectors thus span the tangent plane to the surface

$$
S:=\boldsymbol{\theta}(\bar{\omega})
$$

at the point $\boldsymbol{\theta}(y)$, and the unit vector

$$
\mathbf{a}_{3}(y):=\frac{\mathbf{a}_{1}(y) \wedge \mathbf{a}_{2}(y)}{\left|\mathbf{a}_{1}(y) \wedge \mathbf{a}_{2}(y)\right|}
$$

is normal to $S$ at the point $\boldsymbol{\theta}(y)$. The three vectors $\mathbf{a}_{i}(y)$ constitute the covariant basis at the point $\boldsymbol{\theta}(y)$, while the three vectors $\mathbf{a}^{i}(y)$ defined by the relations

$$
\mathbf{a}^{i}(y) \cdot \mathbf{a}_{j}(y)=\delta_{j}^{i},
$$

where $\delta_{j}^{i}$ is the Kronecker symbol, constitute the contravariant basis at the point $\boldsymbol{\theta}(y) \in S$. Note that $\mathbf{a}^{3}(y)=\mathbf{a}_{3}(y)$ and that the vectors $\mathbf{a}^{\alpha}(y)$ are also in the tangent plane to $S$ at $\boldsymbol{\theta}(y)$. As a consequence, any vector field $\boldsymbol{\eta}: \omega \rightarrow \mathbb{R}^{3}$ can be decomposed over either of these bases as

$$
\boldsymbol{\eta}=\eta_{i} \mathbf{a}^{i}=\eta^{i} \mathbf{a}_{i}
$$

where the coefficients $\eta_{i}$ and $\eta^{i}$ are respectively the covariant and the contravariant components of $\boldsymbol{\eta}$.

The covariant and contravariant components $a_{\alpha \beta}$ and $a^{\alpha \beta}$ of the first fundamental form of $S$, the Christoffel symbols $\Gamma_{\alpha \beta}^{\sigma}$, and the covariant and mixed components $b_{\alpha \beta}$ and $b_{\alpha}^{\beta}$ of the second fundamental form of $S$ are then defined by letting:

$$
\begin{gathered}
a_{\alpha \beta}:=\mathbf{a}_{\alpha} \cdot \mathbf{a}_{\beta}, \quad a^{\alpha \beta}:=\mathbf{a}^{\alpha} \cdot \mathbf{a}^{\beta}, \quad \Gamma_{\alpha \beta}^{\sigma}:=\mathbf{a}^{\sigma} \cdot \partial_{\beta} \mathbf{a}_{\alpha}, \\
b_{\alpha \beta}:=\mathbf{a}^{3} \cdot \partial_{\beta} \mathbf{a}_{\alpha}, \quad b_{\alpha}^{\beta}:=a^{\beta \sigma} b_{\sigma \alpha} .
\end{gathered}
$$

The area element along $S$ is $\sqrt{a} d y$, where

$$
a:=\operatorname{det}\left(a_{\alpha \beta}\right) .
$$

Note that one also has $\sqrt{a}=\left|\mathbf{a}_{1} \wedge \mathbf{a}_{2}\right|$.

The derivatives of the vector fields $\mathbf{a}_{i}$ can be expressed in terms of the Christoffel symbols and of the second fundamental form by means of the equations of Gauss and Weingarten:

$$
\begin{aligned}
& \partial_{\alpha} \mathbf{a}_{\beta}=\Gamma_{\alpha \beta}^{\nu} \mathbf{a}_{\nu}+b_{\alpha \beta} \mathbf{a}^{3}, \\
& \partial_{\alpha} \mathbf{a}_{3}=-b_{\alpha}^{\nu} \mathbf{a}_{\nu} .
\end{aligned}
$$

Likewise, the derivatives of the vector fields $\mathbf{a}^{j}$ satisfy

$$
\begin{aligned}
& \partial_{\alpha} \mathbf{a}^{\tau}=-\Gamma_{\alpha \nu}^{\tau} \mathbf{a}^{\nu}+b_{\alpha}^{\tau} \mathbf{a}^{3}, \\
& \partial_{\alpha} \mathbf{a}^{3}=-b_{\alpha \nu} \mathbf{a}^{\nu} .
\end{aligned}
$$

These equations, combined with the symmetry of the second derivatives of the vector field $\mathbf{a}_{\alpha}$ (i.e., $\partial_{\tau}\left(\partial_{\sigma} \mathbf{a}_{\alpha}\right)=\partial_{\sigma}\left(\partial_{\tau} \mathbf{a}_{\alpha}\right)$ ), imply that

$$
\begin{aligned}
& \left(\partial_{\tau} \Gamma_{\sigma \alpha}^{\nu}+\Gamma_{\sigma \alpha}^{\mu} \Gamma_{\tau \mu}^{\nu}-b_{\sigma \alpha} b_{\tau}^{\nu}\right) \mathbf{a}_{\nu}+\left(\partial_{\tau} b_{\sigma \alpha}+\Gamma_{\sigma \alpha}^{\mu} b_{\tau \mu}\right) \mathbf{a}_{3} \\
& \quad=\left(\partial_{\sigma} \Gamma_{\tau \alpha}^{\nu}+\Gamma_{\tau \alpha}^{\mu} \Gamma_{\sigma \mu}^{\nu}-b_{\tau \alpha} b_{\sigma}^{\nu}\right) \mathbf{a}_{\nu}+\left(\partial_{\sigma} b_{\tau \alpha}+\Gamma_{\tau \alpha}^{\mu} b_{\sigma \mu}\right) \mathbf{a}_{3} .
\end{aligned}
$$


These relations are equivalent to the Gauss and Codazzi-Mainardi equations, namely,

$$
\begin{aligned}
& R_{\cdot \alpha \sigma \tau}^{\nu}=b_{\tau \alpha} b_{\sigma}^{\nu}-b_{\sigma \alpha} b_{\tau}^{\nu}, \\
& \partial_{\sigma} b_{\tau \alpha}-\partial_{\tau} b_{\sigma \alpha}+\Gamma_{\tau \alpha}^{\mu} b_{\sigma \mu}-\Gamma_{\sigma \alpha}^{\mu} b_{\tau \mu}=0
\end{aligned}
$$

where

$$
R_{\cdot \alpha \sigma \tau}^{\nu}:=\partial_{\sigma} \Gamma_{\tau \alpha}^{\nu}-\partial_{\tau} \Gamma_{\sigma \alpha}^{\nu}+\Gamma_{\tau \alpha}^{\mu} \Gamma_{\sigma \mu}^{\nu}-\Gamma_{\sigma \alpha}^{\mu} \Gamma_{\tau \mu}^{\nu}
$$

are the mixed components of the Riemann curvature tensor associated with the metric $\left(a_{\alpha \beta}\right)$. If $R_{\cdot \cdot \sigma \tau}^{\nu \beta}:=a^{\alpha \beta} R_{\cdot \alpha \sigma \tau}^{\nu}$, then one can see that all these functions vanish, save for $R_{\cdot{ }^{12}}^{12}$. This function is the Gaussian curvature of the surface $S$, given by

$$
R_{\cdot 12}^{12}=\frac{\operatorname{det}\left(b_{\alpha \beta}\right)}{\operatorname{det}\left(a_{\alpha \beta}\right)} .
$$

We will see that the sign of the Gaussian curvature plays an important rôle in the two-dimensional theory of shells.

\subsection{Geometry of A Shell}

Let the set $\omega \subset \mathbb{R}^{2}$ and the mapping $\boldsymbol{\theta}: \bar{\omega} \rightarrow \mathbb{R}^{3}$ be as in Section 2.1. In what follows, the surface $S=\boldsymbol{\theta}(\bar{\omega})$ will be identified with the middle surface of a shell before deformation occurs, i.e., $S$ is the middle surface of the reference configuration of the shell. The coordinates $y_{1}, y_{2}$, of the points $y \in \bar{\omega}$ constitute a system of "two-dimensional" curvilinear coordinates for describing the middle surface of the reference configuration of the shell.

More specifically, consider an elastic shell with middle surface $S=\boldsymbol{\theta}(\bar{\omega})$ and (constant) thickness $2 \varepsilon>0$, i.e., an elastic body whose reference configuration is the set $\left\{\hat{\Omega}^{\varepsilon}\right\}^{-}:=\boldsymbol{\Theta}\left(\bar{\Omega}^{\varepsilon}\right)$, where (cf. Figure 2.2-1)

$$
\Omega^{\varepsilon}:=\omega \times(-\varepsilon, \varepsilon) \text { and } \boldsymbol{\Theta}\left(y, x_{3}^{\varepsilon}\right):=\boldsymbol{\theta}(y)+x_{3}^{\varepsilon} \mathbf{a}_{3}(y) \text { for all }\left(y, x_{3}^{\varepsilon}\right) \in \bar{\Omega}^{\varepsilon} .
$$

The more general case of shells with variable thickness or with a middle surface described by several charts (such as an ellipsoid or a torus) can also be dealt with; see, e.g., Busse [16] and S. Mardare [67].

Naturally, this definition makes sense physically only if the mapping $\Theta$ is globally injective on the set $\bar{\Omega}^{\varepsilon}$. Fortunately, this is indeed the case if the immersion $\boldsymbol{\theta}$ is itself globally injective on the set $\bar{\omega}$ and $\varepsilon$ is small enough, according to the following result (due to Ciarlet [20], Theorem 3.1-1).

Theorem 2.2-1. Let $\omega$ be a domain in $\mathbb{R}^{2}$, let $\boldsymbol{\theta} \in \mathcal{C}^{3}\left(\bar{\omega} ; \mathbb{R}^{3}\right)$ be an injective immersion, and let $\Theta: \bar{\omega} \times \mathbb{R} \rightarrow \mathbb{R}^{3}$ be defined by

$$
\boldsymbol{\Theta}\left(y, x_{3}\right):=\boldsymbol{\theta}(y)+x_{3} \mathbf{a}_{3}(y) \text { for all }\left(y, x_{3}\right) \in \bar{\omega} \times \mathbb{R} .
$$

Then there exists $\varepsilon>0$ such that the mapping $\Theta$ is a $\mathcal{C}^{2}$-diffeomorphism from $\bar{\omega} \times[-\varepsilon, \varepsilon]$ onto $\boldsymbol{\Theta}(\bar{\omega} \times[-\varepsilon, \varepsilon])$ and $\operatorname{det}\left(\boldsymbol{g}_{1}, \boldsymbol{g}_{2}, \boldsymbol{g}_{3}\right)>0$ in $\bar{\omega} \times[-\varepsilon, \varepsilon]$, where $\boldsymbol{g}_{i}:=\partial_{i} \boldsymbol{\Theta}$.

Proof. The assumed regularity on $\boldsymbol{\theta}$ implies that $\Theta \in \mathcal{C}^{2}\left(\bar{\omega} \times[-\varepsilon, \varepsilon] ; \mathbb{R}^{3}\right)$ for any $\varepsilon>0$. The relations

$$
\boldsymbol{g}_{\alpha}=\partial_{\alpha} \boldsymbol{\Theta}=\mathbf{a}_{\alpha}+x_{3} \partial_{\alpha} \mathbf{a}_{3} \text { and } \boldsymbol{g}_{3}=\partial_{3} \boldsymbol{\Theta}=\mathbf{a}_{3}
$$

imply that

$$
\left.\operatorname{det}\left(\boldsymbol{g}_{1}, \boldsymbol{g}_{2}, \boldsymbol{g}_{3}\right)\right|_{x_{3}=0}=\operatorname{det}\left(\mathbf{a}_{1}, \mathbf{a}_{2}, \mathbf{a}_{3}\right)>0 \text { in } \bar{\omega} .
$$

Hence $\operatorname{det}\left(\boldsymbol{g}_{1}, \boldsymbol{g}_{2}, \boldsymbol{g}_{3}\right)>0$ on $\bar{\omega} \times[-\varepsilon, \varepsilon]$ if $\varepsilon>0$ is small enough. 
Therefore, the implicit function theorem can be applied if $\varepsilon$ is small enough: It shows that, locally, the mapping $\boldsymbol{\Theta}$ is a $\mathcal{C}^{2}$-diffeomorphism: Given any $y \in \bar{\omega}$, there exist a neighborhood $U(y)$ of $y$ in $\bar{\omega}$ and $\varepsilon(y)>0$ such that $\boldsymbol{\Theta}$ is a $\mathcal{C}^{2}$ diffeomorphism from the set $U(y) \times[-\varepsilon(y), \varepsilon(y)]$ onto $\boldsymbol{\Theta}(U(y) \times[-\varepsilon(y), \varepsilon(y)])$. See, e.g., Schwartz [81], Chapter 3 (the proof of the implicit function theorem, which is almost invariably given for functions defined over open sets, can be easily extended to functions defined over closures of domains, such as the sets $\bar{\omega} \times[-\varepsilon, \varepsilon]$; see, e.g., Stein $[82])$.

To establish that the mapping $\boldsymbol{\Theta}: \bar{\omega} \times[-\varepsilon, \varepsilon] \rightarrow \mathbb{R}^{3}$ is injective provided $\varepsilon>0$ is small enough, we proceed by contradiction: If this property is false, there exist $\varepsilon_{n}>0,\left(y^{n}, x_{3}^{n}\right)$, and $\left(\widetilde{y}^{n}, \widetilde{x}_{3}^{n}\right), n \geq 0$, such that

$$
\begin{gathered}
\varepsilon_{n} \rightarrow 0 \text { as } n \rightarrow \infty, \quad y^{n} \in \bar{\omega}, \quad \widetilde{y}^{n} \in \bar{\omega}, \quad\left|x_{3}^{n}\right| \leq \varepsilon_{n}, \quad\left|\widetilde{x}_{3}^{n}\right| \leq \varepsilon_{n}, \\
\left(y^{n}, x_{3}^{n}\right) \neq\left(\widetilde{y}^{n}, \widetilde{x}_{3}^{n}\right) \text { and } \boldsymbol{\Theta}\left(y^{n}, x_{3}^{n}\right)=\boldsymbol{\Theta}\left(\widetilde{y}^{n}, \widetilde{x}_{3}^{n}\right) .
\end{gathered}
$$

Since the set $\bar{\omega}$ is compact, there exist $y \in \bar{\omega}$ and $\widetilde{y} \in \bar{\omega}$, and there exists an increasing function $\sigma: \mathbb{N} \rightarrow \mathbb{N}$ such that

$$
y^{\sigma(n)} \rightarrow y, \quad \widetilde{y}^{\sigma(n)} \rightarrow \widetilde{y}, \quad x_{3}^{\sigma(n)} \rightarrow 0, \quad \widetilde{x}_{3}^{\sigma(n)} \rightarrow 0 \text { as } n \rightarrow \infty .
$$

Hence

$$
\boldsymbol{\theta}(y)=\lim _{n \rightarrow \infty} \boldsymbol{\Theta}\left(y^{\sigma(n)}, x_{3}^{\sigma(n)}\right)=\lim _{n \rightarrow \infty} \boldsymbol{\Theta}\left(\widetilde{y}^{\sigma(n)}, \widetilde{x}_{3}^{\sigma(n)}\right)=\boldsymbol{\theta}(\widetilde{y}),
$$

by the continuity of the mapping $\boldsymbol{\Theta}$ and thus $y=\widetilde{y}$ since the mapping $\boldsymbol{\theta}$ is injective by assumption. But these properties contradict the local injectivity (noted above) of the mapping $\Theta$. Hence there exists $\varepsilon>0$ such that $\Theta$ is injective on the set $\bar{\omega} \times[-\varepsilon, \varepsilon]$.

In what follows, we assume that $\varepsilon>0$ is small enough so that the conclusions of Theorem 2.2-1 hold. The reference configuration of the considered shell is then defined by

$$
\left\{\hat{\Omega}^{\varepsilon}\right\}^{-}:=\Theta\left(\bar{\Omega}^{\varepsilon}\right),
$$

where $\Omega^{\varepsilon}:=\omega \times(-\varepsilon, \varepsilon)$ and $\hat{\Omega}^{\varepsilon}:=\Theta\left(\Omega^{\varepsilon}\right)$. Let $x^{\varepsilon}=\left(x_{i}^{\varepsilon}\right)$ denote a generic point in the set $\bar{\Omega}^{\varepsilon}$ (hence $x_{\alpha}^{\varepsilon}=y_{\alpha}$ ) and let $\hat{x}^{\varepsilon}=\left(\hat{x}_{i}^{\varepsilon}\right)$ denote a generic point in the reference configuration $\left\{\hat{\Omega}^{\varepsilon}\right\}^{-}$. The reference configuration of the shell can thus be described either in terms of the "three-dimensional" curvilinear coordinates $y_{1}, y_{2}, x_{3}^{\varepsilon}$, or in terms of the Cartesian coordinates $\hat{x}_{1}^{\varepsilon}, \hat{x}_{2}^{\varepsilon}, \hat{x}_{3}^{\varepsilon}$, of the same point $\hat{x}^{\varepsilon}=\boldsymbol{\Theta}\left(x^{\varepsilon}\right) \in$ $\left\{\hat{\Omega}^{\varepsilon}\right\}^{-}$.

To distinguish functions and vector fields defined in Cartesian coordinates from the corresponding functions and vector fields defined in curvilinear coordinates, we henceforth adopt the following convention of notation: Any function or vector field defined on $\hat{\Omega}^{\varepsilon}$ is denoted by letters surmounted by a hat (e.g., $\hat{g}^{\varepsilon}$ is a function defined on $\hat{\Omega}^{\varepsilon}, \hat{f}^{\varepsilon}$ is a vector field defined on $\hat{\Omega}^{\varepsilon}$, etc.). The corresponding functions and vector fields defined in curvilinear coordinates are then denoted by the same letters, but without the hat (e.g., $g^{\varepsilon}$ is the function defined on $\Omega^{\varepsilon}$ by $g^{\varepsilon}\left(x^{\varepsilon}\right)=\hat{g}^{\varepsilon}\left(\hat{x}^{\varepsilon}\right)$ for all $x^{\varepsilon} \in \Omega^{\varepsilon}, \boldsymbol{f}^{\varepsilon}$ is the vector field defined on $\Omega^{\varepsilon}$ by $\boldsymbol{f}^{\varepsilon}\left(x^{\varepsilon}\right)=\hat{\boldsymbol{f}}^{\varepsilon}\left(\hat{x}^{\varepsilon}\right)$ for all $x^{\varepsilon} \in \Omega^{\varepsilon}$, etc., the points $\hat{x}^{\varepsilon}$ and $x^{\varepsilon}$ being related by $\left.\hat{x}^{\varepsilon}=\mathbf{\Theta}\left(x^{\varepsilon}\right)\right)$.

Let $\partial_{i}^{\varepsilon}:=\partial / \partial x_{i}^{\varepsilon}$ (hence $\partial / \partial x_{\alpha}^{\varepsilon}=\partial / \partial y_{\alpha}$ ) and let $\hat{\partial}_{i}^{\varepsilon}:=\partial / \partial \hat{x}_{i}^{\varepsilon}$. For each $x^{\varepsilon} \in \bar{\Omega}^{\varepsilon}$, the three linearly independent vectors $\boldsymbol{g}_{i}^{\varepsilon}\left(x^{\varepsilon}\right):=\partial_{i}^{\varepsilon} \boldsymbol{\Theta}\left(x^{\varepsilon}\right)$ constitute the covariant basis at the point $\Theta\left(x^{\varepsilon}\right)$, and the three (likewise linearly independent) vectors 
$\boldsymbol{g}^{j, \varepsilon}\left(x^{\varepsilon}\right)$ defined by the relations $\boldsymbol{g}^{j, \varepsilon}\left(x^{\varepsilon}\right) \cdot \boldsymbol{g}_{i}^{\varepsilon}\left(x^{\varepsilon}\right)=\delta_{i}^{j}$ constitute the contravariant basis at the same point. As a consequence, any vector field $\boldsymbol{u}^{\varepsilon}: \Omega^{\varepsilon} \rightarrow \mathbb{R}^{3}$ can be decomposed over either basis as

$$
\boldsymbol{u}^{\varepsilon}=u_{i}^{\varepsilon} \boldsymbol{g}^{i, \varepsilon}=u^{i, \varepsilon} \boldsymbol{g}_{i}^{\varepsilon},
$$

where the coefficients $u_{i}^{\varepsilon}$ and $u^{i, \varepsilon}$ are respectively the covariant and the contravariant components of $\boldsymbol{u}^{\varepsilon}$.

The functions $g_{i j}^{\varepsilon}\left(x^{\varepsilon}\right):=\boldsymbol{g}_{i}^{\varepsilon}\left(x^{\varepsilon}\right) \cdot \boldsymbol{g}_{j}^{\varepsilon}\left(x^{\varepsilon}\right)$ and $g^{i j, \varepsilon}\left(x^{\varepsilon}\right):=\boldsymbol{g}^{i, \varepsilon}\left(x^{\varepsilon}\right) \cdot \boldsymbol{g}^{j, \varepsilon}\left(x^{\varepsilon}\right)$ are respectively the covariant and contravariant components of the metric tensor induced by the immersion $\boldsymbol{\Theta}$. The volume element in $\boldsymbol{\Theta}\left(\bar{\Omega}^{\varepsilon}\right)$ is then $\sqrt{g^{\varepsilon}} d x^{\varepsilon}$, where

$$
g^{\varepsilon}:=\operatorname{det}\left(g_{i j}^{\varepsilon}\right) .
$$

For details about these notions of three-dimensional differential geometry, see Ciarlet [23, Sections 1.1-1.3]

FiguRE 2.2-1. The reference configuration of an elastic shell. Let $\omega$ be a domain in $\mathbb{R}^{2}$, let $\Omega^{\varepsilon}=\omega \times(-\varepsilon, \varepsilon)$, let $\boldsymbol{\theta} \in \mathcal{C}^{3}\left(\bar{\omega} ; \mathbb{R}^{3}\right)$ be an immersion, and let the mapping $\Theta: \bar{\Omega}^{\varepsilon} \rightarrow \mathbb{R}^{3}$ be defined by $\boldsymbol{\Theta}\left(y, x_{3}^{\varepsilon}\right)=$ $\boldsymbol{\theta}(y)+x_{3}^{\varepsilon} \mathbf{a}_{3}(y)$ for all $\left(y, x_{3}^{\varepsilon}\right) \in \bar{\Omega}^{\varepsilon}$. Then the mapping $\boldsymbol{\Theta}$ is globally injective on $\bar{\Omega}^{\varepsilon}$ if the immersion $\boldsymbol{\theta}$ is globally injective on $\bar{\omega}$ and $\varepsilon>0$ is small enough (Theorem 2.2-1). In this case, the set $\boldsymbol{\Theta}\left(\bar{\Omega}^{\varepsilon}\right)$ may be viewed as the reference configuration of an elastic shell with thickness $2 \varepsilon$ and middle surface $S=\boldsymbol{\theta}(\bar{\omega})$. The coordinates $\left(y_{1}, y_{2}, x_{3}^{\varepsilon}\right)$ of an arbitrary point $x^{\varepsilon} \in \bar{\Omega}^{\varepsilon}$ are then viewed as curvilinear coordinates of the point $\widehat{x}^{\varepsilon}=\mathbf{\Theta}\left(x^{\varepsilon}\right)$ of the reference configuration of the shell. 


\subsection{The Three-Dimensional Shell EQUATIOnS}

In this section, we consider an elastic shell whose reference configuration is $\left\{\hat{\Omega}^{\varepsilon}\right\}^{-}:=\boldsymbol{\Theta}\left(\bar{\Omega}^{\varepsilon}\right)$ (see Section 2.2), and we make the following assumptions.

The shell is subjected to applied body forces given by their densities $\hat{\boldsymbol{f}}^{\varepsilon}: \hat{\Omega}^{\varepsilon} \rightarrow$ $\mathbb{R}^{3}$ (this means that $\hat{\boldsymbol{f}}^{\varepsilon} d \hat{x}^{\varepsilon}$ is the body force applied to the volume $d \hat{x}^{\varepsilon}$ at each $\left.\hat{x}^{\varepsilon} \in \hat{\Omega}^{\varepsilon}\right)$. For ease of exposition, we assume that there are no applied surface forces.

The shell is subjected to a homogeneous boundary condition of place along the portion $\boldsymbol{\Theta}\left(\gamma_{0} \times[-\varepsilon, \varepsilon]\right)$ of its lateral face $\boldsymbol{\Theta}(\partial \omega \times[-\varepsilon, \varepsilon])$, where $\gamma_{0}$ is a measurable subset of the boundary $\partial \omega$ that satisfies length $\gamma_{0}>0$. This means that the displacement field of the shell vanishes on the set $\boldsymbol{\Theta}\left(\gamma_{0} \times[-\varepsilon, \varepsilon]\right)$.

The shell is made of a homogeneous hyperelastic material, thus characterized by a stored energy function (see Section 1.8)

$$
\hat{W}: \mathbb{M}^{3} \rightarrow \mathbb{R} .
$$

Such a shell problem can thus be modeled by means of a minimization problem (Section 1.8), which is expressed in Cartesian coordinates, in the sense that all functions appearing in the integrands depend on three variables, the Cartesian coordinates $\hat{x}^{\varepsilon}=\left(\hat{x}_{i}^{\varepsilon}\right)$ of a point in the reference configuration $\left\{\hat{\Omega}^{\varepsilon}\right\}^{-}$of the shell. We now recast this problem in terms of the curvilinear coordinates $x^{\varepsilon}=\left(x_{i}^{\varepsilon}\right)$ describing the reference configuration $\left\{\hat{\Omega}^{\varepsilon}\right\}^{-}=\Theta\left(\bar{\Omega}^{\varepsilon}\right)$ of the same shell. This will be the natural point of departure for the two-dimensional approch to shell theory described in the next sections.

More specifically, the minimization problem consists in finding a minimizer $\hat{\boldsymbol{\Phi}}^{\varepsilon}$ : $\left\{\hat{\Omega}^{\varepsilon}\right\}^{-} \rightarrow \mathbb{R}^{3}$ of the functional $\hat{J}^{\varepsilon}$ (see Section 1.8) defined by

$$
\hat{J}^{\varepsilon}\left(\hat{\mathbf{\Psi}}^{\varepsilon}\right):=\int_{\hat{\Omega}^{\varepsilon}} \hat{W}\left(\hat{\boldsymbol{\nabla}}^{\varepsilon} \hat{\mathbf{\Psi}}^{\varepsilon}\right) d \hat{x}^{\varepsilon}-\int_{\hat{\Omega}^{\varepsilon}} \hat{\boldsymbol{f}}^{\varepsilon} \cdot \hat{\mathbf{\Psi}}^{\varepsilon} d \hat{x}^{\varepsilon}
$$

over a set of smooth enough vector fields $\hat{\boldsymbol{\Psi}}^{\varepsilon}=\left\{\hat{\Omega}^{\varepsilon}\right\}^{-} \rightarrow \mathbb{R}^{3}$ satisfying $\hat{\boldsymbol{\Psi}}^{\varepsilon}\left(\hat{x}^{\varepsilon}\right)=\hat{x}^{\varepsilon}$ for all $\hat{x}^{\varepsilon} \in \boldsymbol{\Theta}\left(\gamma_{0} \times[-\varepsilon, \varepsilon]\right)$. Recall that the functional $\hat{J}^{\varepsilon}$ is the total energy of the shell.

This minimization problem can be transformed into a minimization problem posed over the set $\bar{\Omega}^{\varepsilon}$, i.e., expressed in terms of the "natural" curvilinear coordinates of the shell, the unknown $\boldsymbol{\Phi}^{\varepsilon}: \bar{\Omega}^{\varepsilon} \rightarrow \mathbb{R}^{3}$ of this new problem being defined by

$$
\boldsymbol{\Phi}^{\varepsilon}\left(x^{\varepsilon}\right)=\hat{\boldsymbol{\Phi}}^{\varepsilon}\left(\hat{x}^{\varepsilon}\right) \text { for all } \hat{x}^{\varepsilon}=\boldsymbol{\Theta}\left(x^{\varepsilon}\right), x^{\varepsilon} \in \bar{\Omega}^{\varepsilon} .
$$

If $\varepsilon>0$ is small enough, the mapping $\Theta$ is a $\mathcal{C}^{1}$-diffeomorphism of $\bar{\Omega}^{\varepsilon}$ onto its image $\left\{\hat{\Omega}^{\varepsilon}\right\}^{-}=\boldsymbol{\Theta}\left(\bar{\Omega}^{\varepsilon}\right)$ and $\operatorname{det}\left(\boldsymbol{\nabla}^{\varepsilon} \boldsymbol{\Theta}\right)>0$ in $\bar{\Omega}^{\varepsilon}$ (Theorem 2.2-1). The formula for changing variables in multiple integrals then shows that $\boldsymbol{\Phi}^{\varepsilon}$ is a minimizer of the functional $J^{\varepsilon}$ defined by

$$
\begin{aligned}
J^{\varepsilon}\left(\boldsymbol{\Psi}^{\varepsilon}\right):= & \int_{\Omega^{\varepsilon}} \hat{W}\left(\boldsymbol{\nabla} \boldsymbol{\Psi}^{\varepsilon}\left(x^{\varepsilon}\right)\left(\boldsymbol{\nabla}^{\varepsilon} \boldsymbol{\Theta}\left(x^{\varepsilon}\right)\right)^{-1}\right) \operatorname{det} \boldsymbol{\nabla}^{\varepsilon} \boldsymbol{\Theta} d x^{\varepsilon} \\
& -\int_{\Omega^{\varepsilon}} \boldsymbol{f}^{\varepsilon}\left(x^{\varepsilon}\right) \cdot \boldsymbol{\Psi}^{\varepsilon}\left(x^{\varepsilon}\right) \operatorname{det} \boldsymbol{\nabla}^{\varepsilon} \boldsymbol{\Theta} d x^{\varepsilon},
\end{aligned}
$$

where the matrix field $\nabla^{\varepsilon} \Psi^{\varepsilon}: \bar{\Omega}^{\varepsilon} \rightarrow \mathbb{M}^{3}$ is defined by $\nabla^{\varepsilon} \boldsymbol{\Psi}^{\varepsilon}=\left(\partial_{j} \psi_{i}^{\varepsilon}\right)$ (cf. Section 1.1) and the vector field $\boldsymbol{f}^{\varepsilon}: \bar{\Omega}^{\varepsilon} \rightarrow \mathbb{R}^{3}$ is defined by

$$
\boldsymbol{f}^{\varepsilon}\left(x^{\varepsilon}\right):=\hat{\boldsymbol{f}}^{\varepsilon}\left(\hat{x}^{\varepsilon}\right) \text { for all } \hat{x}^{\varepsilon}=\boldsymbol{\Theta}\left(x^{\varepsilon}\right), x^{\varepsilon} \in \Omega^{\varepsilon} .
$$


Note that the function $\operatorname{det} \nabla^{\varepsilon} \boldsymbol{\Theta}$ is equal to the function $\sqrt{g^{\varepsilon}}$, where $g^{\varepsilon}=\operatorname{det}\left(g_{i j}^{\varepsilon}\right)$; cf. Section 2.2.

Consider next a linearly elastic shell with Lamé constants $\lambda>0$ and $\mu>$ 0 . In this case, the minimization problem associated with the equations of linearized three-dimensional elasticity (Section 1.4) consists in finding a minimizer $\hat{\boldsymbol{\Phi}}^{\varepsilon}:\left\{\hat{\Omega}^{\varepsilon}\right\}^{-} \rightarrow \mathbb{R}^{3}$ over a set of smooth enough vector fields $\hat{\mathbf{\Psi}}^{\varepsilon}=\left\{\hat{\Omega}^{\varepsilon}\right\}^{-} \rightarrow \mathbb{R}^{3}$ satisfying $\hat{\mathbf{\Psi}}^{\varepsilon}\left(\hat{x}^{\varepsilon}\right)=\hat{x}^{\varepsilon}$ for all $\hat{x}^{\varepsilon} \in \boldsymbol{\Theta}\left(\gamma_{0} \times[-\varepsilon, \varepsilon]\right)$ of the functional $\hat{J}^{\varepsilon}$ defined by

$$
\hat{J}^{\varepsilon}\left(\hat{\mathbf{\Psi}}^{\varepsilon}\right):=\int_{\hat{\Omega}^{\varepsilon}} \hat{W}\left(\hat{\nabla}^{\varepsilon} \hat{\Psi}^{\varepsilon}\right) d \hat{x}^{\varepsilon}-\int_{\hat{\Omega}^{\varepsilon}} \hat{\boldsymbol{f}}^{\varepsilon} \cdot \hat{\mathbf{\Psi}}^{\varepsilon} d \hat{x}^{\varepsilon},
$$

where

$$
\hat{W}(\boldsymbol{F})=\frac{\lambda}{8}\left(\operatorname{tr}\left(\boldsymbol{F}^{T}+\boldsymbol{F}-2 \boldsymbol{I}\right)\right)^{2}+\frac{\mu}{4}\left\|\boldsymbol{F}^{T}+\boldsymbol{F}-2 \boldsymbol{I}\right\|^{2} \text { for all } \boldsymbol{F} \in \mathbb{M}^{3}
$$

(this stored energy function for a linearly elsatic material easily follows from the equations of linearized three-dimensional elasticity given in Section 1.4). Its expression shows that the functional $\hat{J}^{\varepsilon}$ is well defined if $\hat{\boldsymbol{\Psi}}^{\varepsilon} \in H^{1}\left(\hat{\Omega}^{\varepsilon} ; \mathbb{R}^{3}\right)$.

As in the nonlinear case, this minimization problem can be recast in curvilinear coordinates. As such, it consists in finding a minimizer $\boldsymbol{\Phi}^{\varepsilon}: \bar{\Omega}^{\varepsilon} \rightarrow \mathbb{R}^{3}$ over the set of all vector fields $\boldsymbol{\Psi}^{\varepsilon} \in H^{1}\left(\bar{\Omega}^{\varepsilon} ; \mathbb{R}^{3}\right)$ satisfying $\boldsymbol{\Psi}^{\varepsilon}=\boldsymbol{\Theta}$ on $\gamma_{0} \times[-\varepsilon, \varepsilon]$ of the functional $J^{\varepsilon}$ defined by

$$
J^{\varepsilon}\left(\boldsymbol{\Psi}^{\varepsilon}\right)=\int_{\Omega^{\varepsilon}} \hat{W}\left(\boldsymbol{\nabla}^{\varepsilon} \boldsymbol{\Psi}^{\varepsilon}\left(\boldsymbol{\nabla}^{\varepsilon} \boldsymbol{\Theta}\right)^{-1}\right) \sqrt{g^{\varepsilon}} d x^{\varepsilon}-\int_{\Omega^{\varepsilon}} \boldsymbol{f}^{\varepsilon} \cdot \boldsymbol{\Psi}^{\varepsilon} \sqrt{g^{\varepsilon}} d x^{\varepsilon} .
$$

As usual in linearized elasticity, it is more convenient to express this energy in terms of the displacement field $\boldsymbol{u}^{\varepsilon}: \bar{\Omega}^{\varepsilon} \rightarrow \mathbb{R}^{3}$, defined by

$$
\boldsymbol{\Phi}^{\varepsilon}\left(x^{\varepsilon}\right)=\boldsymbol{\Theta}\left(x^{\varepsilon}\right)+\boldsymbol{u}^{\varepsilon}\left(x^{\varepsilon}\right) \text { for all } x^{\varepsilon} \in \bar{\Omega}^{\varepsilon} .
$$

Likewise, let $\boldsymbol{v}^{\varepsilon}: \bar{\Omega}^{\varepsilon} \rightarrow \mathbb{R}^{3}$ be such that $\Psi^{\varepsilon}=\boldsymbol{\Theta}+\boldsymbol{v}^{\varepsilon}$. Then a straightforward calculation shows that

$$
\hat{W}\left(\boldsymbol{\nabla}^{\varepsilon} \boldsymbol{\Psi}^{\varepsilon}\left(\boldsymbol{\nabla}^{\varepsilon} \boldsymbol{\Theta}\right)^{-1}\right)=A^{i j k \ell, \varepsilon} e_{i j}^{\varepsilon}\left(\boldsymbol{v}^{\varepsilon}\right) e_{k \ell}^{\varepsilon}\left(\boldsymbol{v}^{\varepsilon}\right),
$$

where

$$
\begin{aligned}
A^{i j k \ell, \varepsilon}:=\lambda g^{i j, \varepsilon} g^{k \ell, \varepsilon}+\mu\left(g^{i k, \varepsilon} g^{j \ell, \varepsilon}+g^{i \ell, \varepsilon} g^{j k, \varepsilon}\right), \\
e_{i j}^{\varepsilon}\left(\boldsymbol{v}^{\varepsilon}\right):=\frac{1}{2}\left(\partial_{i}^{\varepsilon} \boldsymbol{v}^{\varepsilon} \cdot \boldsymbol{g}_{j}^{\varepsilon}+\partial_{j}^{\varepsilon} \boldsymbol{v}^{\varepsilon} \cdot \boldsymbol{g}_{i}^{\varepsilon}\right) .
\end{aligned}
$$

The functions $A^{i j k \ell, \varepsilon}$ and $e_{i j}^{\varepsilon}\left(\boldsymbol{u}^{\varepsilon}\right)$ denote respectively the contravariant components of the three-dimensional elasticity tensor in curvilinear coordinates, and the covariant components of the linearized strain tensor associated with the displacement field $\boldsymbol{v}^{\varepsilon}$. It is then easy to see that $\boldsymbol{u}^{\varepsilon}$ is a minimizer over the vector space

$$
\boldsymbol{V}\left(\Omega^{\varepsilon}\right):=\left\{\boldsymbol{u}^{\varepsilon}=u_{i}^{\varepsilon} \boldsymbol{g}^{i, \varepsilon} ; u_{i}^{\varepsilon} \in H^{1}\left(\Omega^{\varepsilon}\right), u_{i}^{\varepsilon}=0 \text { on } \gamma_{0} \times(-\varepsilon, \varepsilon)\right\},
$$

of the functional $J^{\varepsilon}$ defined by

$$
J^{\varepsilon}\left(\boldsymbol{u}^{\varepsilon}\right):=\frac{1}{2} \int_{\Omega^{\varepsilon}} A^{i j k \ell, \varepsilon} e_{i j}^{\varepsilon}\left(\boldsymbol{u}^{\varepsilon}\right) e_{k \ell}^{\varepsilon}\left(\boldsymbol{u}^{\varepsilon}\right) \sqrt{g^{\varepsilon}} d x^{\varepsilon}-\int_{\Omega^{\varepsilon}} \boldsymbol{f}^{\varepsilon} \cdot \boldsymbol{u}^{\varepsilon} \sqrt{g^{\varepsilon}} d x^{\varepsilon} .
$$

This minimization problem will be used in Section 2.6 as a point of departure for deriving two-dimensional linear shell models. 


\subsection{THE TWO-DIMENSIONAL APPROACH TO SHELL THEORY}

In a two-dimensional approach, the above minimization problems of Section 2.3 are "replaced" by a, presumably much simpler, two-dimensional problem, this time "posed over the middle surface $S$ of the shell". This means that the new unknown should be now the deformation $\varphi: \bar{\omega} \rightarrow \mathbb{R}^{3}$ of the points of the middle surface $S=\boldsymbol{\theta}(\bar{\omega})$, or, equivalently, the displacement field $\boldsymbol{\zeta}: \bar{\omega} \rightarrow \mathbb{R}^{3}$ of the points of the same surface $S$ (the deformation and the displacement fields are related by the equation $\boldsymbol{\varphi}=\boldsymbol{\theta}+\boldsymbol{\zeta}$ ); cf. Figure 2.4-1.

FIGURE 2.4-1. An elastic shell modeled as a two-dimensional problem. For $\varepsilon>0$ "small enough" and data of ad hoc orders of magnitude, the three-dimensional shell problem is "replaced" by a "two-dimensional shell problem". This means that the new unknowns are the three covariant components $\zeta_{i}: \bar{\omega} \rightarrow \mathbb{R}$ of the displacement field $\zeta_{i} \mathbf{a}^{i}: \bar{\omega} \rightarrow \mathbb{R}^{3}$ of the points of the middle surface $S=\boldsymbol{\theta}(\bar{\omega})$. In this process, the "three-dimensional" boundary conditions on $\Gamma_{0}$ need to be replaced by ad hoc "two-dimensional" boundary conditions on $\gamma_{0}$. For instance, the "boundary conditions of clamping" $\zeta_{i}=\partial_{\nu} \zeta_{3}=0$ on $\gamma_{0}$ (used in Koiter's linear equations; cf. Section 2.8) mean that the points of, and the tangent spaces to, the deformed and undeformed middle surfaces coincide along the set $\boldsymbol{\theta}\left(\gamma_{0}\right)$.

The two-dimensional approach to shell theory yield a variety of two-dimensional shell models, which can be classified in two categories (the same classification applies for both nonlinear and linearized shell models):

A first category of two-dimensional models are those that are obtained from the three-dimesional equations of shells "by letting $\varepsilon$ go to zero". Depending on the data (geometry of the middle surface of the shell, boundary conditions imposed on 
the displacement fields, applied forces) one obtains either a membrane shell model, or a flexural shell model, also called a bending shell model. A brief description of these models and of their derivation is given in Sections 2.5 and 2.6.

A second category of two-dimensional models are those that are obtained from the three-dimensional model by restricting the range of admissible deformations and stresses by means of specific a priori assumptions that are supposed to take into account the "smallness" of the thickness (e.g., the Cosserat assumptions, the Kirchhoff-Love assumptions, etc.). A variety of two-dimensional models of shells are obtained in this fashion, as, e.g., those of Koiter, Naghdi, etc. A detailed description of Koiter's model is given in Sections 2.7 and 2.8.

\subsection{Nonlinear Shell MOdels OBtained By $\Gamma$-CONVERGENCE}

Remarkable achievements in the asymptotic analysis of nonlinearly elastic shells are due to Le Dret \& Raoult [64] and to Friesecke, James, Mora \& Müller [49], who gave the first (and only ones to this date) rigorous proofs of convergence as the thickness approaches zero. In so doing, they extended to shells the analysis that they had successfully applied to nonlinearly elastic plates in Le Dret \& Raoult [62] and Friesecke, James \& Müller [48].

We begin with the asymptotic analysis of nonlinearly elastic membrane shells. $\mathrm{H}$. Le Dret and A. Raoult showed that a subsequence of deformations that minimize (or rather "almost minimize" in a sense explained below) the scaled three-dimensional energies weakly converges in $W^{1, p}\left(\Omega ; \mathbb{R}^{3}\right)$ as $\varepsilon \rightarrow 0$ (the number $p>1$ is governed by the growth properties of the stored energy function). They showed in addition that the weak limit minimizes a "membrane" energy that is the $\Gamma$-limit of the (appropriately scaled) energies. We now give an abridged account of their analysis.

Let $\omega$ be a domain in $\mathbb{R}^{2}$ with boundary $\gamma$ and let $\boldsymbol{\theta} \in \mathcal{C}^{2}\left(\bar{\omega} ; \mathbb{R}^{3}\right)$ be an injective mapping such that the two vectors $\boldsymbol{a}_{\alpha}(y)=\partial_{\alpha} \boldsymbol{\theta}(y)$ are linearly independent at all points $y=\left(y_{\alpha}\right) \in \bar{\omega}$.

Consider a family of elastic shells with the same middle surface $S=\boldsymbol{\theta}(\bar{\omega})$ and whose thickness $2 \varepsilon>0$ approaches zero. The reference configuration of each shell is thus the image $\Theta\left(\bar{\Omega}^{\varepsilon}\right) \subset \mathbb{R}^{3}$ of the set $\bar{\Omega}^{\varepsilon} \subset \mathbb{R}^{3}$ through a mapping $\Theta: \bar{\Omega}^{\varepsilon} \rightarrow \mathbb{R}^{3}$ defined in Section 2.2 .

By Theorem 2.2-1, if the injective mapping $\boldsymbol{\theta}: \bar{\omega} \rightarrow \mathbb{R}^{3}$ is smooth enough, the mapping $\Theta: \bar{\Omega}^{\varepsilon} \rightarrow \mathbb{R}^{3}$ is also injective for $\varepsilon>0$ small enough and $y_{1}, y_{2}, x_{3}^{\varepsilon}$ then constitute the "natural" curvilinear coordinates for describing each reference configuration $\Theta\left(\bar{\Omega}^{\varepsilon}\right)$.

Assume that all the shells in the family are made of the same hyperelastic homogeneous material (see Section 1.8), satisfying the following properties :

The stored energy function $\hat{W}: \mathbb{M}^{3} \rightarrow \mathbb{R}$ of the hyperelastic material satisfies the following assumptions: There exist constants $C>0, \alpha>0, \beta \in \mathbb{R}$, and $1<p<\infty$ such that

$$
\begin{aligned}
|\hat{W}(\boldsymbol{F})| & \leq C\left(1+|\boldsymbol{F}|^{p}\right) \quad \text { for all } \boldsymbol{F} \in \mathbb{M}^{3}, \\
\hat{W}(\boldsymbol{F}) & \geq \alpha|\boldsymbol{F}|^{p}+\beta \text { for all } \boldsymbol{F} \in \mathbb{M}^{3}, \\
|\hat{W}(\boldsymbol{F})-\hat{W}(\boldsymbol{G})| & \leq C\left(1+|\boldsymbol{F}|^{p-1}+|\boldsymbol{G}|^{p-1}\right)|\boldsymbol{F}-\boldsymbol{G}| \\
& \text { for all } \boldsymbol{F}, \boldsymbol{G} \in \mathbb{M}^{3} .
\end{aligned}
$$


It can be verified that the stored energy function of a St Venant-Kirchhoff material, which is given by

$$
\hat{W}(\boldsymbol{F})=\frac{\mu}{4} \operatorname{tr}\left(\boldsymbol{F}^{T} \boldsymbol{F}-\boldsymbol{I}\right)^{2}+\frac{\lambda}{8}\left\{\operatorname{tr}\left(\boldsymbol{F}^{T} \boldsymbol{F}-\boldsymbol{I}\right)\right\}^{2},
$$

satisfies such inequalities with $p=4$.

Remark. By contrast, the stored energy function of a linearly elastic material, which is given by

$$
\hat{W}(\boldsymbol{F})=\frac{\mu}{4}\left\|\boldsymbol{F}+\boldsymbol{F}^{T}-2 \boldsymbol{I}\right\|^{2}+\frac{\lambda}{8}\left\{\operatorname{tr}\left(\boldsymbol{F}^{T}+\boldsymbol{F}-2 \boldsymbol{I}\right)\right\}^{2},
$$

where $\|\boldsymbol{F}\|:=\left\{\operatorname{tr} \boldsymbol{F}^{T} \boldsymbol{F}\right\}^{1 / 2}$, satisfies the first inequality with $p=2$, but not the second one.

It is further assumed that, for each $\varepsilon>0$, the shells are subjected in their interior to applied body forces of density $\hat{\boldsymbol{f}}^{\varepsilon}=\hat{f}_{i}^{\varepsilon} \boldsymbol{g}^{i, \varepsilon}: \hat{\Omega}^{\varepsilon} \rightarrow \mathbb{R}^{3}$ per unit volume, where $\hat{f}_{i}^{\varepsilon} \in L^{q}\left(\hat{\Omega}^{\varepsilon}\right)$ and $\frac{1}{p}+\frac{1}{q}=1$, and that these densities do not depend on the unknown deformation. Applied surface forces on the "upper" and "lower" faces of the shells could be likewise considered, but are omitted for simplicity; see in this respect Le Dret \& Raoult [64] who consider a pressure load, an example of applied surface force that depends on the unknown deformation.

Finally, it is assumed that each shell is subjected to a boundary condition of place along its entire lateral face $\boldsymbol{\Theta}(\gamma \times[-\varepsilon, \varepsilon])$, where $\gamma:=\partial \omega$, i.e., that the displacement vanishes there.

The three-dimensional problem is then posed as a minimization problem in terms of the unknown deformation field

$$
\boldsymbol{\Phi}^{\varepsilon}\left(x^{\varepsilon}\right):=\boldsymbol{\Theta}\left(x^{\varepsilon}\right)+\boldsymbol{u}^{\varepsilon}\left(x^{\varepsilon}\right), x^{\varepsilon} \in \bar{\Omega}^{\varepsilon},
$$

of the reference configuration, where $\boldsymbol{u}^{\varepsilon}: \bar{\Omega}^{\varepsilon} \rightarrow \mathbb{R}^{3}$ is its displacement field (Section 2.3): It consists in finding $\boldsymbol{\Phi}^{\varepsilon}$ such that

$$
\begin{gathered}
\boldsymbol{\Phi}^{\varepsilon} \in \mathcal{M}\left(\Omega^{\varepsilon}\right) \text { and } J^{\varepsilon}\left(\boldsymbol{\Phi}^{\varepsilon}\right)=\inf _{\boldsymbol{\Psi}^{\varepsilon} \in \mathcal{M}\left(\Omega^{\varepsilon}\right)} J^{\varepsilon}\left(\boldsymbol{\Psi}^{\varepsilon}\right), \text { where } \\
\mathcal{M}\left(\Omega^{\varepsilon}\right):=\left\{\boldsymbol{\Psi}^{\varepsilon} \in W^{1, p}\left(\Omega^{\varepsilon} ; \mathbb{R}^{3}\right) ; \boldsymbol{\Psi}^{\varepsilon}=\boldsymbol{\Theta} \text { on } \gamma \times[-\varepsilon, \varepsilon]\right\}, \\
J^{\varepsilon}\left(\boldsymbol{\Psi}^{\varepsilon}\right)=\int_{\Omega^{\varepsilon}} \hat{W}\left(\boldsymbol{\nabla}^{\varepsilon} \boldsymbol{\Psi}^{\varepsilon}\left(\boldsymbol{\nabla}^{\varepsilon} \boldsymbol{\Theta}\right)^{-1}\right) \operatorname{det} \boldsymbol{\nabla}^{\varepsilon} \boldsymbol{\Theta} d x^{\varepsilon} \\
\quad-\int_{\Omega^{\varepsilon}} \boldsymbol{f}^{\varepsilon} \cdot \boldsymbol{\Psi}^{\varepsilon} \operatorname{det} \boldsymbol{\nabla}^{\varepsilon} \boldsymbol{\Theta} d x^{\varepsilon} .
\end{gathered}
$$

This minimization problem may have no solution; however, this is not a shortcoming as only the existence of a "diagonal infimizing family", whose existence is always guaranteed, is required in the ensuing analysis (Theorem 2.5-1).

The above minimization problem is then transformed into an analogous one, but now posed over the fixed domain $\Omega:=\omega \times]-1,1\left[\right.$. Let $x=\left(x_{1}, x_{2}, x_{3}\right)$ denote a generic point in $\bar{\Omega}$ and let $\partial_{i}:=\partial / \partial x_{i}$. With each point $x \in \bar{\Omega}$, we associate the point $x^{\varepsilon} \in \bar{\Omega}^{\varepsilon}$ through the bijection

$$
\pi^{\varepsilon}: x=\left(x_{1}, x_{2}, x_{3}\right) \in \bar{\Omega} \rightarrow x^{\varepsilon}=\left(x_{i}^{\varepsilon}\right)=\left(x_{1}, x_{2}, \varepsilon x_{3}\right) \in \bar{\Omega}^{\varepsilon} .
$$

We then define the unknown scaled deformation $\Phi(\varepsilon): \bar{\Omega} \rightarrow \mathbb{R}^{3}$ by letting

$$
\boldsymbol{\Phi}(\varepsilon)(x):=\boldsymbol{\Phi}^{\varepsilon}\left(x^{\varepsilon}\right) \text { for all } x^{\varepsilon}=\pi^{\varepsilon} x, x \in \bar{\Omega} .
$$


Finally, we assume that the applied body forces are of order $\mathcal{O}(1)$ with respect to $\varepsilon$, in the sense that there exists a vector field $f \in \mathrm{E}^{2}\left(\Omega ; \mathbb{R}^{3}\right)$ independent of $\varepsilon$ such that

$$
\boldsymbol{f}^{\varepsilon}\left(x^{\varepsilon}\right)=\boldsymbol{f}(x) \text { for all } x^{\varepsilon}=\pi^{\varepsilon} x, x \in \bar{\Omega} \text {. }
$$

Remark. Should applied surface forces act on the upper and lower faces of the shells, we would then assume that they are of order $O(\varepsilon)$ with respect to $\varepsilon$.

In what follows, the notation $\left(\boldsymbol{b}_{1} ; \boldsymbol{b}_{2} ; \boldsymbol{b}_{3}\right)$ stands for the matrix in $\mathbb{M}^{3}$ whose three column vectors are $\boldsymbol{b}_{1}, \boldsymbol{b}_{2}, \boldsymbol{b}_{3}$ (in this order).

These scalings and assumptions then imply that the scaled deformation $\boldsymbol{\Phi}(\varepsilon)$ satisfies the following minimization problem:

$$
\begin{array}{r}
\boldsymbol{\Phi}(\varepsilon) \in \mathcal{M}(\varepsilon ; \Omega) \text { and } J(\varepsilon)(\boldsymbol{\Phi}(\varepsilon))=\inf _{\boldsymbol{\Psi} \in \mathcal{M}(\varepsilon ; \Omega)} J(\varepsilon)(\boldsymbol{\Psi}), \text { where } \\
\mathcal{M}(\varepsilon ; \Omega):=\left\{\boldsymbol{\Psi} \in W^{1, p}\left(\Omega ; \mathbb{R}^{3}\right) ; \boldsymbol{\Psi}=\boldsymbol{\Phi}_{0}(\varepsilon) \text { on } \gamma \times[-1,1]\right\}, \\
J(\varepsilon)(\boldsymbol{\Psi}):=\int_{\Omega} \hat{W}\left(\left(\partial_{1} \boldsymbol{\Psi} ; \partial_{2} \mathbf{\Psi} ; \frac{1}{\varepsilon} \partial_{3} \boldsymbol{\Psi}\right)(\boldsymbol{G}(\varepsilon))^{-1}\right) \operatorname{det} \boldsymbol{G}(\varepsilon) d x \\
\quad-\int_{\Omega} \boldsymbol{f} \cdot \boldsymbol{\psi} \operatorname{det} \boldsymbol{G}(\varepsilon) d x,
\end{array}
$$

where the vector field $\boldsymbol{\Phi}_{0}(\varepsilon): \bar{\Omega} \rightarrow \mathbb{R}^{3}$ is defined for each $\varepsilon>0$ by

$$
\boldsymbol{\Phi}_{0}(\varepsilon)(x):=\mathbf{\Theta}\left(x^{\varepsilon}\right) \text { for all } x^{\varepsilon}=\pi^{\varepsilon} x, x \in \bar{\Omega},
$$

and the matrix field $\boldsymbol{G}(\varepsilon): \bar{\Omega} \rightarrow \mathbb{M}^{3}$ is defined by

$$
\boldsymbol{G}(\varepsilon)(x):=\boldsymbol{\nabla}^{\varepsilon} \boldsymbol{\Theta}\left(x^{\varepsilon}\right) \text { for all } x^{\varepsilon}=\pi^{\varepsilon} x, x \in \bar{\Omega} .
$$

The scaled displacement

$$
\boldsymbol{u}(\varepsilon):=\boldsymbol{\Phi}(\varepsilon)-\boldsymbol{\Phi}_{0}(\varepsilon)
$$

therefore satisfies the following minimization problem:

$$
\begin{aligned}
\boldsymbol{u}(\varepsilon) \in W\left(\Omega ; \mathbb{R}^{3}\right) \text { and } J(\varepsilon)(\boldsymbol{u}(\varepsilon)) & =\inf _{\boldsymbol{v} \in W\left(\Omega ; \mathbb{R}^{3}\right)} J(\varepsilon)(\boldsymbol{v}), \text { where } \\
W\left(\Omega ; \mathbb{R}^{3}\right) & :=\left\{\boldsymbol{v} \in W^{1, p}\left(\Omega ; \mathbb{R}^{3}\right) ; \boldsymbol{v}=\mathbf{0} \text { on } \gamma \times[-1,1]\right\} \\
J(\varepsilon)(\boldsymbol{v}) & :=\int_{\Omega} \hat{W}\left(\boldsymbol{I}+\left(\partial_{1} \boldsymbol{v} ; \partial_{2} \boldsymbol{v} ; \frac{1}{\varepsilon} \partial_{3} \boldsymbol{v}\right)(\boldsymbol{G}(\varepsilon))^{-1}\right) \operatorname{det} \boldsymbol{G}(\varepsilon) d x \\
& -\int_{\Omega} \boldsymbol{f} \cdot\left(\boldsymbol{\Phi}_{0}(\varepsilon)+\boldsymbol{v}\right) \operatorname{det} \boldsymbol{G}(\varepsilon) d x .
\end{aligned}
$$

Central to the ensuing result of convergence is the notion of quasiconvexity, due to Morrey [71, 72] (an account of its importance in the calculus of variations is provided in Dacorogna [38, Chap 5]): Let $\mathbb{M}^{m \times n}$ denote the space of all real matrices with $m$ rows and $n$ columns; a function $\hat{W}: \mathbb{M}^{m \times n} \rightarrow \mathbb{R}$ is quasiconvex if, for all bounded open subsets $U \subset \mathbb{R}^{n}$, all $\boldsymbol{F} \in \mathbb{M}^{m \times n}$, and all $\boldsymbol{\xi}=\left(\xi_{i}\right)_{i=1}^{m} \in W_{0}^{1, \infty}\left(U ; \mathbb{R}^{m}\right)$,

$$
\hat{W}(\boldsymbol{F}) \leq \frac{1}{\text { meas } U} \int_{U} \hat{W}(\boldsymbol{F}+\boldsymbol{\nabla} \boldsymbol{\xi}(x)) d x,
$$

where $\boldsymbol{\nabla} \boldsymbol{\xi}$ denotes the matrix $\left(\partial_{j} \xi_{i}\right) \in \mathbb{M}^{m \times n}$. Given any function $\hat{W}: \mathbb{M}^{m \times n} \rightarrow \mathbb{R}$, its quasiconvex envelope $\mathcal{Q} \hat{W}: \mathbb{M}^{m \times n} \rightarrow \mathbb{R}$ is the function defined by

$$
\mathcal{Q} \hat{W}:=\sup \left\{\hat{X}: \mathbb{M}^{m \times n} \rightarrow \mathbb{R} ; \hat{X} \text { is quasi-convex and } \hat{X} \leq \hat{W}\right\} .
$$


Remark. An illuminating instance of actual computation of a quasiconvex envelope is found in Le Dret \& Raoult [63], who explicitly determine the quasiconvex envelope of the stored energy function of a St Venant-Kirchhoff material.

Also central to the ensuing analysis is the notion of $\Gamma$-convergence, a powerful theory initiated by De Giorgi [40,41] (see also De Giorgi \& Franzoni [43]); an illuminating introduction is found in De Giorgi \& Dal Maso [42] and thorough treatments are given in the books of Attouch [8] and Dal Maso [39]. As shown by Acerbi, Buttazzo \& Percivale [1] for nonlinearly elastic strings, by Le Dret \& Raoult $[62,64]$ for nonlinearly elastic planar membranes and membrane shells, by Friesecke, James \& Müller [48] for nonlinearly elastic flexural plates, and by Friesecke, James, Mora \& Müller [49] for nonlinearly elastic flexural shells, this approach has thus far provided the only known convergence theorems for justifying lower-dimensional nonlinear theories of elastic bodies. See also Ciarlet [19, Section 1.11] for an application to linearly elastic plates.

We then recall the fundamental definition underlying this theory: Let $V$ be a metric space and let $J(\varepsilon): V \rightarrow \mathbb{R}$ be functionals defined for all $\varepsilon>0$. The family $(J(\varepsilon))_{\varepsilon>0}$ is said to $\Gamma$-converge as $\varepsilon \rightarrow 0$ if there exists a functional $J: V \rightarrow$ $\mathbb{R} \cup\{+\infty\}$, called the $\Gamma$-limit of the functionals $J(\varepsilon)$, such that

$$
v(\varepsilon) \rightarrow v \text { as } \varepsilon \rightarrow 0 \Rightarrow J(v) \leq \liminf _{\varepsilon \rightarrow 0} J(\varepsilon)(v(\varepsilon)),
$$

on the one hand and, given any $v \in V$, there exist $v(\varepsilon) \in V, \varepsilon>0$, such that

$$
v(\varepsilon) \rightarrow v \text { as } \varepsilon \rightarrow 0 \text { and } J(v)=\lim _{\varepsilon \rightarrow 0} J(\varepsilon)(v(\varepsilon)),
$$

on the other.

As a preparation to the application of $\Gamma$-convergence theory, the scaled energies $J(\varepsilon): \mathcal{M}(\Omega) \rightarrow \mathbb{R}$ found above are first extended to the larger space $L^{p}\left(\Omega ; \mathbb{R}^{3}\right)$ by letting

$$
\tilde{J}(\varepsilon)(\boldsymbol{v})=\left\{\begin{array}{l}
J(\varepsilon)(\boldsymbol{v}) \text { if } \boldsymbol{v} \in \mathcal{M}(\Omega), \\
+\infty \text { if } \boldsymbol{v} \in L^{p}\left(\Omega ; \mathbb{R}^{3}\right) \text { but } \boldsymbol{v} \notin \mathcal{M}(\Omega) .
\end{array}\right.
$$

Such an extension, customary in $\Gamma$-convergence theory, has inter alia the advantage of "incorporating" the boundary condition into the extended functional.

Le Dret \& Raoult [64] then establish that the family $(\tilde{J}(\varepsilon))_{\varepsilon>0}$ of extended energies $\Gamma$-converges as $\varepsilon \rightarrow 0$ in $L^{p}\left(\Omega ; \mathbb{R}^{3}\right)$ and that its $\Gamma$-limit can be computed by means of quasiconvex envelopes.

More precisely, their analysis leads to the following remarkable convergence theorem, where the limit minimization problems are directly posed as two-dimensional problems (part c)); this is licit since the solutions of these limit problems do not depend on the transverse variable (part (b)). Note that, while minimizers of $J(\varepsilon)$ over $\mathcal{M}(\Omega)$ need not exist, the existence of a "diagonal infimizing family" in the sense understood below is always guaranteed because $\inf _{\boldsymbol{v} \in \mathcal{M}(\Omega)} J(\varepsilon)(\boldsymbol{v})>-\infty$.

In what follows, the notation $\left(\boldsymbol{b}_{1} ; \boldsymbol{b}_{2}\right)$ stands for the matrix in $\mathbb{M}^{3 \times 2}$ with $\boldsymbol{b}_{1}, \boldsymbol{b}_{2}$ (in this order) as its column vectors and $\sqrt{a} d y$ denotes as usual the area element along the surface $S$.

Theorem 2.5-1. Assume that the applied body forces are of order $\mathcal{O}(1)$ with respect to $\varepsilon$, and that there exist $C>0, \alpha>0, \beta \in \mathbb{R}$, and $1<p<\infty$ such that the stored 
energy function $\hat{W}: \mathbb{M}^{3} \rightarrow \mathbb{R}$ satisfies the following growth conditions:

$$
\begin{aligned}
|\hat{W}(\boldsymbol{F})| & \leq C\left(1+|\boldsymbol{F}|^{p}\right) & & \text { for all } \boldsymbol{F} \in \mathbb{M}^{3}, \\
\hat{W}(\boldsymbol{F}) & \geq \alpha|\boldsymbol{F}|^{p}+\beta & & \text { for all } \boldsymbol{F} \in \mathbb{M}^{3}, \\
|\hat{W}(\boldsymbol{F})-\hat{W}(\boldsymbol{G})| & \leq C\left(1+|\boldsymbol{F}|^{p-1}+|\boldsymbol{G}|^{p-1}\right)|\boldsymbol{F}-\boldsymbol{G}| & & \text { for all } \boldsymbol{F}, \boldsymbol{G} \in \mathbb{M}^{3} .
\end{aligned}
$$

Let the space $\mathcal{M}(\Omega)$ be defined by

$$
\mathcal{M}(\Omega):=\left\{\boldsymbol{v} \in W^{1, p}\left(\Omega ; \mathbb{R}^{3}\right) ; \boldsymbol{v}=\mathbf{0} \text { on } \gamma \times[-1,1]\right\},
$$

and let $(\boldsymbol{u}(\varepsilon))_{\varepsilon>0}$ be a "diagonal infimizing family" of the scaled energies, i.e., a family that satisfies

$$
\boldsymbol{u}(\varepsilon) \in \mathcal{M}(\Omega) \text { and } J(\varepsilon)(\boldsymbol{u}(\varepsilon)) \leq \inf _{\boldsymbol{v} \in \mathcal{M}(\Omega)} J(\varepsilon)(\boldsymbol{v})+h(\varepsilon) \text { for all } \varepsilon>0,
$$

where $h$ is any positive function that satisfies $h(\varepsilon) \rightarrow 0$ as $\varepsilon \rightarrow 0$. Then:

(a) The family $(\boldsymbol{u}(\varepsilon))_{\varepsilon>0}$ lies in a weakly compact subset of the space $W^{1, p}\left(\Omega ; \mathbb{R}^{3}\right)$.

(b) The limit $\boldsymbol{u} \in \mathcal{M}(\Omega)$ as $\varepsilon \rightarrow 0$ of any weakly convergent subsequence of $(\boldsymbol{u}(\varepsilon))_{\varepsilon>0}$ satisfies $\partial_{3} \boldsymbol{u}=\mathbf{0}$ in $\Omega$ and is thus independent of the transverse variable.

(c) The vector field $\boldsymbol{\zeta}:=\frac{1}{2} \int_{-1}^{1} \boldsymbol{u} d x_{3}$ satisfies the following minimization problem:

$$
\boldsymbol{\zeta} \in W_{0}^{1, p}\left(\omega ; \mathbb{R}^{3}\right) \text { and } j_{M}(\boldsymbol{\zeta})=\inf _{\boldsymbol{\eta} \in W_{0}^{1, p}\left(\omega ; \mathbb{R}^{3}\right)} j_{M}(\boldsymbol{\eta}),
$$

where

$$
\begin{aligned}
& j_{M}(\boldsymbol{\eta}):=2 \int_{\omega} \mathcal{Q} \hat{W}_{M}\left(y,\left(\boldsymbol{a}_{1}+\partial_{1} \boldsymbol{\eta} ; \boldsymbol{a}_{2}+\partial_{2} \boldsymbol{\eta}\right)\right) \sqrt{a} d y-\int_{\omega}\left\{\int_{-1}^{1} \boldsymbol{f} d x_{3}\right\} \cdot \boldsymbol{\eta} \sqrt{a} d y, \\
& \hat{W}_{M}\left(y,\left(\boldsymbol{b}_{1} ; \boldsymbol{b}_{2}\right)\right):=\inf _{\boldsymbol{b}_{3} \in \mathbb{R}^{3}} \hat{W}\left(\left(\boldsymbol{b}_{1} ; \boldsymbol{b}_{2} ; \boldsymbol{b}_{3}\right) \boldsymbol{G}^{-1}(y)\right) \text { for all }\left(y,\left(\boldsymbol{b}_{1} ; \boldsymbol{b}_{2}\right)\right) \in \bar{\omega} \times \mathbb{M}^{3 \times 2}, \\
& \boldsymbol{G}(y):=\left(\boldsymbol{a}_{1}(y) ; \boldsymbol{a}_{2}(y) ; \boldsymbol{a}_{3}(y)\right),
\end{aligned}
$$

the vectors $\boldsymbol{a}_{i}(y)$ forming for each $y \in \bar{\omega}$ the covariant basis at the point $\boldsymbol{\theta}(y) \in S$, and $\mathcal{Q} \hat{W}_{0}(y, \cdot)$ denotes for each $y \in \bar{\omega}$ the quasiconvex envelope of $\hat{W}_{0}(y, \cdot)$.

It remains to de-scale the vector field $\boldsymbol{\zeta}$. In view of the scalings performed on the deformations, we are naturally led to defining for each $\varepsilon>0$ the limit displacement field $\boldsymbol{\zeta}^{\varepsilon}: \bar{\omega} \rightarrow \mathbb{R}^{3}$ of the middle surface $S$ by

$$
\zeta^{\varepsilon}:=\zeta
$$

It is then immediately verified that $\boldsymbol{\zeta}^{\varepsilon}$ satisfies the following minimization problem (the notations are those of Theorem 2.5-1):

$$
\begin{aligned}
\boldsymbol{\zeta}^{\varepsilon} \in W_{0}^{1, p}\left(\omega ; \mathbb{R}^{3}\right) \text { and } j_{M}^{\varepsilon}\left(\boldsymbol{\zeta}^{\varepsilon}\right)=\inf _{\boldsymbol{\eta} \in W_{0}^{1, p}\left(\omega ; \mathbb{R}^{3}\right)} j_{M}^{\varepsilon}(\boldsymbol{\eta}), \text { where } \\
\begin{aligned}
j_{M}^{\varepsilon}(\boldsymbol{\eta})=2 \varepsilon \int_{\omega} \mathcal{Q} \hat{W}_{M}\left(y,\left(\boldsymbol{a}_{1}+\partial_{1} \boldsymbol{\eta} ;\right.\right. & \left.\left.\boldsymbol{a}_{2}+\partial_{2} \boldsymbol{\eta}\right)\right) \sqrt{a} d y \\
& -\int_{\omega}\left\{\int_{-\varepsilon}^{\varepsilon} \boldsymbol{f}^{\varepsilon} d x_{3}^{\varepsilon}\right\} \cdot \boldsymbol{\eta} \sqrt{a} d y .
\end{aligned}
\end{aligned}
$$


The unknown $\boldsymbol{\eta}$ in the above minimization problem appears only by means of its first-order partial derivatives $\partial_{\alpha} \boldsymbol{\eta}$ in the stored energy function

$$
\boldsymbol{\eta} \in W^{1, p}\left(\omega ; \mathbb{R}^{3}\right) \rightarrow \varepsilon \mathcal{Q} \hat{W}_{F}\left(\cdot,\left(\boldsymbol{a}_{1}+\partial_{1} \boldsymbol{\eta} ; \boldsymbol{a}_{2}+\partial_{2} \boldsymbol{\eta}\right)\right)
$$

found in the integrand of the energy $j_{M}^{\varepsilon}$.

Assume that the original stored energy function is frame-indifferent, in the sense that

$$
\hat{W}(\mathbf{R} \boldsymbol{F})=\hat{W}(\boldsymbol{F}) \text { for all } \boldsymbol{F} \in \mathbb{M}^{3} \text { and } \mathbf{R} \in \mathbb{O}_{+}^{3} .
$$

This relation is stronger than the usual one, which holds only for $\boldsymbol{F} \in \mathbb{M}^{3}$ with $\operatorname{det} \boldsymbol{F}>0$ (see Ciarlet [18, Theorem 4.2-1]); it is, however, verified by the kinds of stored energy functions to which the present analysis applies, e.g., that of a St Venant-Kirchhoff material. Under this stronger assumption, Le Dret \& Raoult [64, Theorem 10] establish the crucial properties that the stored energy function found in $j_{M}^{\varepsilon}$, once expressed as a function of the points of $S$, is frame-indifferent and that it depends only on the metric of the deformed middle surface. For this reason, this theory is a frame-indifferent, nonlinear "membrane" shell theory.

It is remarkable that the stored energy function found in $j_{M}^{\varepsilon}$ can be explicitly computed when the original three-dimensional stored energy function is that of a St Venant-Kirchhoff material; see Le Dret \& Raoult [64, Section 6].

Again for a St Venant-Kirchhoff material, Genevey [50] has furthermore shown that, when the singular values of the $3 \times 2$ matrix fields $\left(\partial_{\alpha} \eta_{i}\right)$ associated with a field $\boldsymbol{\eta}=\eta_{i} \mathbf{a}^{i}$ belong to an appropriate compact subset of $\mathbb{R}^{2}$ (which can be explicitely identified), the expression $j_{M}^{\varepsilon}(\boldsymbol{\eta})$ takes the simpler form

$j_{M}^{\varepsilon}(\boldsymbol{\eta})=\frac{\varepsilon}{8} \int_{\omega} a^{\alpha \beta \sigma \tau}\left(a_{\sigma \tau}(\boldsymbol{\eta})-a_{\sigma \tau}\right)\left(a_{\alpha \beta}(\boldsymbol{\eta})-a_{\alpha \beta}\right) \sqrt{a} d y-\int_{\omega}\left\{\int_{-\varepsilon}^{\varepsilon} \boldsymbol{f}^{\varepsilon} d x_{3}^{\varepsilon}\right\} \cdot \boldsymbol{\eta} \sqrt{a} d y$,

where

$$
\begin{aligned}
a^{\alpha \beta \sigma \tau} & :=\frac{4 \lambda \mu}{\lambda+2 \mu} a^{\alpha \beta} a^{\sigma \tau}+2 \mu\left(a^{\alpha \sigma} a^{\beta \tau}+a^{\alpha \tau} a^{\beta \sigma}\right), \\
a_{\alpha \beta}(\boldsymbol{\eta}) & :=\partial_{\alpha}\left(\boldsymbol{\theta}+\eta_{i} \mathbf{a}^{i}\right) \cdot \partial_{\beta}\left(\boldsymbol{\theta}+\eta_{j} \mathbf{a}^{j}\right) .
\end{aligned}
$$

This is precisely the expression of $j_{M}^{\varepsilon}(\boldsymbol{\eta})$ that was found by Miara [69] to hold "for all fields $\boldsymbol{\eta}$ " (i.e., without any restriction on the fields $\boldsymbol{\eta}$ such as that found by Genevey [50]), by means of a formal asymptotic analysis. This observation thus provides a striking example where the limit equations found by a formal asymptotic analysis "do not always coincide" with those found by means of a rigorous convergence theorem.

Le Dret \& Raoult [64, Section 6] have further shown that, if the stored energy function is frame-indifferent and satisfies $\hat{W}(\boldsymbol{F}) \geq \hat{W}(\boldsymbol{I})$ for all $\boldsymbol{F} \in \mathbb{M}^{3}$ (as does the stored energy function of a St Venant-Kirchhoff material), then the corresponding shell energy is constant under compression. This result has the striking consequence that "nonlinear membrane shells offer no resistance to crumpling. This is an empirical fact, witnessed by anyone who ever played with a deflated balloon" (to quote H. Le Dret and A. Raoult).

We now turn our attention to the asymptotic analysis, by means of $\Gamma$-convergence theory, of nonlinearly elastic flexural shells. In its principle, the approach is essentially the same (although more delicate) as that used for deriving the nonlinear membrane shell equations. There are, however, two major differences regarding the assumptions that are made at the onset of the asymptotic analysis. 
A first difference is that the applied body forces are now assumed to be of order $\mathcal{O}\left(\varepsilon^{2}\right)$ with respect to $\varepsilon$ (instead of $\mathcal{O}(1)$ ), in the sense that there exists a vector field $\boldsymbol{f} \in L^{2}\left(\Omega ; \mathbb{R}^{3}\right)$ independent of $\varepsilon$ such that

$$
\boldsymbol{f}^{\varepsilon}\left(x^{\varepsilon}\right)=\varepsilon^{2} \boldsymbol{f}(x) \text { for all } x^{\varepsilon}=\pi^{\varepsilon} x \in \bar{\Omega} .
$$

A second difference (without any counterpart for membrane shells) is that the set denoted $\mathcal{M}_{F}(\omega)$ in the next theorem contains other fields than $\boldsymbol{\theta}$ (the interpretation of this key assumption is briefly commented upon after the theorem).

Under these assumptions, Friesecke, James, Mora \& Müller [49] have proved the following result. The notations $\mathbf{a}_{3}(\boldsymbol{\psi}), a_{\alpha \beta}(\boldsymbol{\psi})$, and $b_{\alpha \beta}(\boldsymbol{\psi})$ used in the next statement are self-explanatory: Given an arbitrary vector field $\boldsymbol{\psi} \in H^{2}\left(\omega ; \mathbb{R}^{3}\right)$,

$$
\mathbf{a}_{3}(\boldsymbol{\psi}):=\frac{\partial_{1} \boldsymbol{\psi} \wedge \partial_{2} \boldsymbol{\psi}}{\left|\partial_{1} \boldsymbol{\psi} \wedge \partial_{2} \boldsymbol{\psi}\right|}, a_{\alpha \beta}(\boldsymbol{\psi}):=\partial_{\alpha} \boldsymbol{\psi} \cdot \partial_{\beta} \boldsymbol{\psi}, b_{\alpha \beta}(\boldsymbol{\psi}):=-\partial_{\alpha} \mathbf{a}_{3}(\boldsymbol{\psi}) \cdot \partial_{\beta} \boldsymbol{\psi}
$$

Theorem 2.5-2. Assume that the applied body forces are of order $\mathcal{O}\left(\varepsilon^{2}\right)$ with respect to $\varepsilon$. Assume in addition that the stored energy function $\hat{W}: \mathbb{M}^{3} \rightarrow \mathbb{R}$ satisfies the following properties: It is measurable and of class $\mathcal{C}^{2}$ in a neighborhood of $\mathbb{O}_{+}^{3}$, it satisfies

$$
\hat{W}(\boldsymbol{I})=0 \text { and } \hat{W}(\boldsymbol{R F})=\hat{W}(\boldsymbol{F}) \text { for all } \boldsymbol{F} \in \mathbb{M}^{3} \text { and } \boldsymbol{R} \in \mathbb{O}_{+}^{3},
$$

and, finally, it satisfies the following growth condition: There exists a constant $C>0$ such that

$$
|\hat{W}(\boldsymbol{F})| \geq C \inf _{\boldsymbol{R} \in \mathbb{O}_{+}^{3}}|\boldsymbol{F}-\boldsymbol{R}|^{2} \text { for all } \boldsymbol{F} \in \mathbb{M}^{3} .
$$

Finally, assume that the set

$$
\begin{aligned}
\mathcal{M}_{F}(\omega):=\left\{\boldsymbol{\psi} \in H^{2}\left(\omega ; \mathbb{R}^{3}\right) ;\right. & a_{\alpha \beta}(\boldsymbol{\psi})=a_{\alpha \beta} \text { in } \omega ; \\
& \left.\boldsymbol{\psi}=\boldsymbol{\theta} \text { and } \mathbf{a}_{3}(\boldsymbol{\psi})=\mathbf{a}_{3} \text { on } \gamma_{0}\right\},
\end{aligned}
$$

contains other vector fields than $\boldsymbol{\theta}$.

Then the scaled energies $J(\varepsilon), \varepsilon>0$, constitute a family that $\Gamma$-converges as $\varepsilon \rightarrow 0$ in the following sense: Any "diagonal infimizing family" (defined as in Theorem 2.5-1) contains a subsequence that strongly converges in $H^{1}\left(\Omega ; \mathbb{R}^{3}\right)$.

Besides, the limit $\boldsymbol{\Phi}$ of any such subsequence is independent of the transverse variable, and the vector field $\boldsymbol{\varphi}:=\frac{1}{2} \int_{-1}^{1} \boldsymbol{\Phi} d x_{3}$ satisfies

$$
\boldsymbol{\varphi} \in \mathcal{M}_{F}(\omega) \text { and } j_{F}(\boldsymbol{\varphi})=\inf _{\boldsymbol{\psi} \in \mathcal{M}_{F}(\omega)} j_{F}(\boldsymbol{\psi}),
$$

where the functional $j_{F}: \mathcal{M}_{F}(\omega) \rightarrow \mathbb{R}$ is defined by

$$
\begin{aligned}
j_{F}(\boldsymbol{\psi})= & \frac{2}{3} \int_{\omega} \hat{W}_{F}\left(y,\left(\left(b_{1 \beta}(\boldsymbol{\psi})-b_{1 \beta}\right) \mathbf{a}^{\beta} ;\left(b_{2 \beta}(\boldsymbol{\psi})-b_{2 \beta}\right) \mathbf{a}^{\beta}\right)\right) \sqrt{a} d y \\
& -\int_{\omega}\left\{\int_{-1}^{1} \boldsymbol{f}_{\varepsilon} d x_{3}\right\} \cdot \boldsymbol{\psi} \sqrt{a} d y
\end{aligned}
$$

where

$$
\begin{aligned}
& \hat{W}_{F}\left(y,\left(\boldsymbol{b}_{1} ; \boldsymbol{b}_{2}\right)\right):=\inf _{\boldsymbol{b}_{3} \in \mathbb{R}^{3}} \frac{1}{2} \frac{\partial^{2} \hat{W}}{\partial \boldsymbol{F}^{2}}(\boldsymbol{I})\left(\left(\boldsymbol{b}_{1} ; \boldsymbol{b}_{2} ; \boldsymbol{b}_{3}\right) \boldsymbol{G}^{-1}(y),\left(\boldsymbol{b}_{1} ; \boldsymbol{b}_{2} ; \boldsymbol{b}_{3}\right) \boldsymbol{G}^{-1}(y)\right) \\
& \quad \text { for all }\left(y,\left(\boldsymbol{b}_{1} ; \boldsymbol{b}_{2}\right)\right) \in \bar{\omega} \times \mathbb{M}^{3 \times 2}, \\
& \boldsymbol{G}(y):=\left(\mathbf{a}_{1}(y) ; \mathbf{a}_{2}(y) ; \mathbf{a}_{3}(y)\right) .
\end{aligned}
$$


The assumption that the set $\mathcal{M}_{F}(\omega)$ contains other vector fields than $\boldsymbol{\theta}$ means that there exist nonzero displacement fields $\eta_{i} \mathbf{a}^{i}$ of the middle surface $\boldsymbol{\theta}(\bar{\omega})$ that are both inextensional, in the sense that the surfaces $\boldsymbol{\theta}(\bar{\omega})$ and $\boldsymbol{\psi}(\bar{\omega})$, where $\boldsymbol{\psi}:=$ $\boldsymbol{\theta}+\eta_{i} \mathbf{a}^{i}$, have the same metric (as reflected by the assumption $a_{\alpha \beta}(\boldsymbol{\psi})=a_{\alpha \beta}$ in $\omega)$, and admissible, in the sense that the points of, and the tangent spaces to, the surfaces $\boldsymbol{\theta}(\bar{\omega})$ and $\boldsymbol{\psi}(\bar{\omega})$ coincide along the set $\boldsymbol{\theta}\left(\gamma_{0}\right)$ (as reflected by the boundary conditions $\boldsymbol{\psi}=\boldsymbol{\theta}$ and $\mathbf{a}_{3}(\boldsymbol{\psi})=\mathbf{a}_{3}$ on $\left.\gamma_{0}\right)$.

It follows that the "de-scaled" unknown deformation $\varphi^{\varepsilon}: \bar{\omega} \rightarrow \mathbb{R}^{3}$ of the middle surface of the shell is a minimizer over the set $\mathcal{M}_{F}(\omega)$ of the functional $j_{F}^{\varepsilon}$ defined by

$$
\begin{aligned}
j_{F}^{\varepsilon}(\boldsymbol{\psi}):= & \frac{2 \varepsilon^{3}}{3} \int_{\omega} \hat{W}_{F}\left(y,\left(\left(b_{1 \beta}(\boldsymbol{\psi})-b_{1 \beta}\right) \mathbf{a}^{\beta} ;\left(b_{2 \beta}(\boldsymbol{\psi})-b_{2 \beta}\right) \mathbf{a}^{\beta}\right)\right) \sqrt{a} d y \\
& -\int_{\omega}\left\{\int_{-\varepsilon}^{\varepsilon} \boldsymbol{f}_{\varepsilon} d x_{3}^{\varepsilon}\right\} \cdot \boldsymbol{\psi} \sqrt{a} d y \text { for all } \boldsymbol{\psi} \in \mathcal{M}_{F}(\omega) .
\end{aligned}
$$

When the original three-dimensional stored energy function is that of a St Venant Kirchhoff material, the expression $j_{F}^{\varepsilon}(\boldsymbol{\psi})$ takes the simpler form

$j_{F}^{\varepsilon}(\boldsymbol{\psi})=\frac{\varepsilon^{3}}{6} \int_{\omega} a^{\alpha \beta \sigma \tau}\left(b_{\sigma \tau}(\boldsymbol{\psi})-b_{\sigma \tau}\right)\left(b_{\alpha \beta}(\boldsymbol{\psi})-b_{\alpha \beta}\right) \sqrt{a} d y-\int_{\omega}\left\{\int_{-\varepsilon}^{\varepsilon} \boldsymbol{f}_{\varepsilon} d x_{3}^{\varepsilon}\right\} \cdot \boldsymbol{\psi} \sqrt{a} d y$,

where

$$
a^{\alpha \beta \sigma \tau}:=\frac{4 \lambda \mu}{\lambda+2 \mu} a^{\alpha \beta} a^{\sigma \tau}+2 \mu\left(a^{\alpha \sigma} a^{\beta \tau}+a^{\alpha \tau} a^{\beta \sigma}\right) .
$$

Interestingly, exactly the same expression $j_{F}^{\varepsilon}(\psi)$ was found for all $\boldsymbol{\psi} \in \mathcal{M}_{F}(\omega)$ by means of a formal asymptotic analysis by Lods \& Miara [65], as the outcome of sometimes exceedingly delicate computations. This observation is thus in sharp contrast with that made for a membrane shell, whose limit equations cannot always be recovered by a formal approach, as noted earlier.

Remark. Although $\Gamma$-convergence automatically provides the existence of a minimizer of the $\Gamma$-limit functional, the existence of a minimizer of the functional $j_{F}$ over the set $\mathcal{M}_{F}(\omega)$ can be also established by means of a direct method of calculus of variations; cf. Ciarlet \& Coutand [24].

\subsection{LINEAR SHELL MODELS OBTAINED BY ASYMPTOTIC ANALYSIS}

In this section, we briefly review the genesis of those two-dimensional linear shell theories that can be found, and rigorously justified, as the outcome of an asymptotic analysis of the equations of three-dimensional linearized elasticity as $\varepsilon \rightarrow 0$.

The asymptotic analysis of elastic shells has been a subject of considerable attention during the past decades. After the landmark attempt of Goldenveizer [53], a major step for linearly elastic shells was achieved by Destuynder [44] in his Doctoral Dissertation, where a convergence theorem for "membrane shells" was "almost proved". Another major step was achieved by Sanchez-Palencia [77], who clearly delineated the kinds of geometries of the middle surface and boundary conditions that yield either two-dimensional membrane, or two-dimensional flexural, equations when the method of formal asymptotic expansions is applied to the variational equations of three-dimensional linearized elasticity (see also Caillerie \& Sanchez-Palencia [17] and Miara \& Sanchez-Palencia [70]). 
Then Ciarlet \& Lods [27, 28] and Ciarlet, Lods \& Miara [31] carried out an asymptotic analysis of linearly elastic shells that covers all possible cases: Under three distinct sets of assumptions on the geometry of the middle surface, on the boundary conditions, and on the order of magnitude of the applied forces, they established convergence theorems in $H^{1}$, in $L^{2}$, or in ad hoc completion spaces, that justify either the linear two-dimensional equations of a "membrane shell", or those of a "generalized membrane shell", or those of a "flexural shell".

More specifically, consider a family of linearly elastic shells of thickness $2 \varepsilon$ that satisfy the following assumptions: All the shells have the same middle surface $S=\boldsymbol{\theta}(\bar{\omega}) \subset \mathbb{R}^{3}$, where $\omega$ is a domain in $\mathbb{R}^{2}$ with boundary $\gamma$, and $\boldsymbol{\theta} \in \mathcal{C}^{3}\left(\bar{\omega} ; \mathbb{R}^{3}\right)$. Their reference configurations are thus of the form $\Theta\left(\bar{\Omega}^{\varepsilon}\right), \varepsilon>0$, where

$$
\Omega^{\varepsilon}:=\omega \times(-\varepsilon, \varepsilon),
$$

and the mapping $\Theta$ is defined by

$$
\boldsymbol{\Theta}\left(y, x_{3}^{\varepsilon}\right):=\boldsymbol{\theta}(y)+x_{3}^{\varepsilon} \mathbf{a}_{3}(y) \text { for all }\left(y, x_{3}^{\varepsilon}\right) .
$$

All the shells in the family are made with the same homogeneous isotropic elastic material and that their reference configurations are natural states. Their elastic material is thus characterized by two Lamé constants $\lambda>0$ and $\mu>0$.

The shells are subjected to body forces and that the corresponding applied body force density is $O\left(\varepsilon^{p}\right)$ with respect to $\varepsilon$, for some ad hoc power $p$ (which will be specified later). This means that, for each $\varepsilon>0$, the contravariant components $f^{i, \varepsilon} \in L^{2}\left(\Omega^{\varepsilon}\right)$ of the body force density $\boldsymbol{f}^{\varepsilon}=f^{i, \varepsilon} \boldsymbol{g}_{i}^{\varepsilon}$ are of the form

$$
\left.f^{i, \varepsilon}\left(y, \varepsilon x_{3}\right)=\varepsilon^{p} f^{i}\left(y, x_{3}\right) \text { for all }\left(y, x_{3}\right) \in \Omega:=\omega \times\right]-1,1[,
$$

and the functions $f^{i} \in L^{2}(\Omega)$ are independent of $\varepsilon$ (surface forces acting on the "upper" and "lower" faces of the shell could be as well taken into account but will not be considered here, for simplicity of exposition). Let then the functions $p^{i, \varepsilon} \in L^{2}(\omega)$ be defined for each $\varepsilon>0$ by

$$
p^{i, \varepsilon}:=\int_{-\varepsilon}^{\varepsilon} f^{i, \varepsilon} d x_{3}^{\varepsilon} \text {. }
$$

Finally, each shell is subjected to a boundary condition of place on the portion $\boldsymbol{\Theta}\left(\gamma_{0} \times[-\varepsilon, \varepsilon]\right)$ of its lateral face, where $\gamma_{0}$ is a fixed portion of $\gamma$, with length $\gamma_{0}>0$.

Then the displacement field of the shell satisfies the following minimization problem associated with the equations of linearized three-dimensional elasticity in curvilinear coordinates (see Section 2.3):

$$
\begin{aligned}
& \boldsymbol{u}^{\varepsilon} \in \boldsymbol{V}\left(\Omega^{\varepsilon}\right) \text { and } J_{\varepsilon}\left(\boldsymbol{u}^{\varepsilon}\right)=\min _{\boldsymbol{v}^{\varepsilon} \in \boldsymbol{V}\left(\Omega^{\varepsilon}\right)} J^{\varepsilon}\left(\boldsymbol{v}^{\varepsilon}\right), \text { where } \\
& J^{\varepsilon}\left(\boldsymbol{v}^{\varepsilon}\right):=\frac{1}{2} \int_{\Omega^{\varepsilon}} A^{i j k \ell, \varepsilon} e_{i j}^{\varepsilon}\left(\boldsymbol{v}^{\varepsilon}\right) e_{k \ell}^{\varepsilon}\left(\boldsymbol{v}^{\varepsilon}\right) \sqrt{g^{\varepsilon}} d x^{\varepsilon}-\int_{\Omega^{\varepsilon}} \boldsymbol{f}^{\varepsilon} \cdot \boldsymbol{v}^{\varepsilon} \sqrt{g^{\varepsilon}} d x^{\varepsilon}, \\
& \boldsymbol{V}\left(\Omega^{\varepsilon}\right):=\left\{\boldsymbol{v}^{\varepsilon}=v_{i}^{\varepsilon} \boldsymbol{g}^{i, \varepsilon} ; v_{i}^{\varepsilon} \in H^{1}(\Omega), v_{i}^{\varepsilon}=0 \text { on } \gamma_{0} \times(-\varepsilon, \varepsilon)\right\} .
\end{aligned}
$$

For each $\varepsilon>0$, this problem has one and only one solution $\boldsymbol{u}^{\varepsilon} \in \boldsymbol{V}(\Omega)$.

For any displacement field $\boldsymbol{\eta}=\eta_{i} \mathbf{a}^{i}: \omega \rightarrow \mathbb{R}^{3}$, let

$$
\gamma_{\alpha \beta}(\boldsymbol{\eta})=\frac{1}{2}\left(\partial_{\beta} \boldsymbol{\eta} \cdot \mathbf{a}_{\alpha}+\partial_{\alpha} \boldsymbol{\eta} \cdot \mathbf{a}_{\beta}\right) \text { and } \rho_{\alpha \beta}(\boldsymbol{\eta})=\left(\partial_{\alpha \beta} \boldsymbol{\eta}-\Gamma_{\alpha \beta}^{\sigma} \partial_{\sigma} \boldsymbol{\eta}\right) \cdot \mathbf{a}_{3}
$$

denote as usual the covariant components of the linearized change of metric, and linearized change of curvature, tensors. 
In Ciarlet, Lods \& Miara [31] it is first assumed that the space of linearized inextensional displacements (introduced by Sanchez-Palencia [75])

$$
\begin{aligned}
\boldsymbol{V}_{F}(\omega):=\left\{\boldsymbol{\eta}=\eta_{i} \mathbf{a}^{i} ;\right. & \eta_{\alpha} \in H^{1}(\omega), \eta_{3} \in H^{2}(\omega) ; \\
& \left.\eta_{i}=\partial_{\nu} \eta_{3}=0 \text { on } \gamma_{0}, \gamma_{\alpha \beta}(\boldsymbol{\eta})=0 \text { in } \omega\right\}
\end{aligned}
$$

contains non-zero functions. This assumption is in fact one in disguise about the geometry of the surface $S$ and on the set $\gamma_{0}$. For instance, it is satisfied if $S$ is a portion of a cylinder and $\boldsymbol{\theta}\left(\gamma_{0}\right)$ is contained in one or two generatrices of $S$, or if $S$ is contained in a plane, in which case the shells are plates.

Under this assumption Ciarlet, Lods \& Miara [31] showed that, if the applied body force density is $O\left(\varepsilon^{2}\right)$ with respect to $\varepsilon$, then

$$
\frac{1}{2 \varepsilon} \int_{-\varepsilon}^{\varepsilon} \boldsymbol{u}^{\varepsilon} d x_{3}^{\varepsilon} \rightarrow \boldsymbol{\zeta} \text { in } H^{1}\left(\omega ; \mathbb{R}^{3}\right) \text { as } \varepsilon \rightarrow 0,
$$

where the limit vector field $\boldsymbol{\zeta}:=\zeta_{i} \mathbf{a}^{i}$ belongs to the space $\boldsymbol{V}_{F}(\omega)$ and satisfies the equations of a linearly elastic "flexural shell", viz.,

$$
\frac{\varepsilon^{3}}{3} \int_{\omega} a^{\alpha \beta \sigma \tau} \rho_{\sigma \tau}(\boldsymbol{\zeta}) \rho_{\alpha \beta}(\boldsymbol{\eta}) \sqrt{a} d y=\int_{\omega} p^{i, \varepsilon} \eta_{i} \sqrt{a} d y
$$

for all $\boldsymbol{\eta}=\eta_{i} \mathbf{a}^{i} \in \boldsymbol{V}_{F}(\omega)$. Observe in passing that the limit $\boldsymbol{\zeta}$ is indeed independent of $\varepsilon$, since both sides of these variational equations are of the same order (viz., $\varepsilon^{3}$ ), because of the assumptions made on the applied forces.

Equivalently, the vector field $\boldsymbol{\zeta}$ satisfies the following constrained minimization problem:

$$
\boldsymbol{\zeta} \in \boldsymbol{V}_{F}(\omega) \text { and } j_{F}^{\varepsilon}(\boldsymbol{\zeta})=\inf j_{F}^{\varepsilon}(\boldsymbol{\eta})
$$

where

$$
j_{F}^{\varepsilon}(\boldsymbol{\eta}):=\frac{1}{2} \int_{\omega} \frac{\varepsilon^{3}}{3} a^{\alpha \beta \sigma \tau} \rho_{\sigma \tau}(\boldsymbol{\eta}) \rho_{\alpha \beta}(\boldsymbol{\eta}) \sqrt{a} d y-\int_{\omega} p^{i, \varepsilon} \eta_{i} \sqrt{a} d y
$$

for all $\boldsymbol{\eta}=\eta_{i} \mathbf{a}^{i} \in \boldsymbol{V}_{F}(\omega)$, where the functions

$$
a^{\alpha \beta \sigma \tau}=\frac{4 \lambda \mu}{(\lambda+2 \mu)} a^{\alpha \beta} a^{\sigma \tau}+2 \mu\left(a^{\alpha \sigma} a^{\beta \tau}+a^{\alpha \tau} a^{\beta \sigma}\right)
$$

are precisely the familiar contravariant components of the shell elasticity tensor.

If $\boldsymbol{V}_{F}(\omega) \neq\{\mathbf{0}\}$, the two-dimensional equations of a linearly elastic "flexural shell" are therefore justified.

If $\boldsymbol{V}_{F}(\omega)=\{\mathbf{0}\}$, the above convergence result still applies. However, the only information it provides is that $\frac{1}{2 \varepsilon} \int_{-\varepsilon}^{\varepsilon} \boldsymbol{u}^{\varepsilon} d x_{3}^{\varepsilon} \rightarrow \mathbf{0}$ in $H^{1}\left(\omega ; \mathbb{R}^{3}\right)$ as $\varepsilon \rightarrow 0$. Hence a more refined asymptotic analysis is needed in this case.

A first instance of such a refinement was given by Ciarlet \& Lods [27], where it was assumed that $\gamma_{0}=\gamma$ and that the surface $S$ is elliptic, in the sense that its Gaussian curvature is $>0$ everywhere. As shown in Ciarlet \& Lods [27] and Ciarlet \& Sanchez-Palencia [35], these two conditions, together with ad hoc regularity assumptions, indeed imply that $\boldsymbol{V}_{F}(\omega)=\{\mathbf{0}\}$.

In this case, Ciarlet \& Lods [28] showed that, if the applied body force density is $O(1)$ with respect to $\varepsilon$, then

$$
\frac{1}{2 \varepsilon} \int_{-\varepsilon}^{\varepsilon} u_{\alpha}^{\varepsilon} d x_{3}^{\varepsilon} \rightarrow \zeta_{\alpha} \text { in } H^{1}(\omega) \text { and } \frac{1}{2 \varepsilon} \int_{-\varepsilon}^{\varepsilon} u_{3}^{\varepsilon} d x_{3}^{\varepsilon} \rightarrow \zeta_{3} \text { in } L^{2}(\omega) \text { as } \varepsilon \rightarrow 0,
$$


where the limit vector field $\boldsymbol{\zeta}:=\zeta_{i} \mathbf{a}^{i}$ belongs to the space

$$
\boldsymbol{V}_{M}(\omega):=\left\{\boldsymbol{\eta}=\eta_{i} \mathbf{a}^{i} ; \eta_{\alpha} \in H_{0}^{1}(\omega), \eta_{3} \in L^{2}(\omega)\right\},
$$

and solves the equations of a linearly elastic "membrane shell", viz.,

$$
\int_{\omega} \varepsilon a^{\alpha \beta \sigma \tau} \gamma_{\sigma \tau}(\boldsymbol{\zeta}) \gamma_{\alpha \beta}(\boldsymbol{\eta}) \sqrt{a} d y=\int_{\omega} p^{i, \varepsilon} \eta_{i} \sqrt{a} d y
$$

for all $\boldsymbol{\eta}=\eta_{i} \mathbf{a}^{i} \in \boldsymbol{V}_{M}(\omega)$, where the functions $a^{\alpha \beta \sigma \tau}, \gamma_{\alpha \beta}(\boldsymbol{\eta}), a$, and $p^{i, \varepsilon}$ have the same meanings as above. If $\gamma_{0}=\gamma$ and $S$ is elliptic, the two-dimensional equations of a linearly elastic "membrane shell" are therefore justified. Observe that the limit $\boldsymbol{\zeta}$ is again independent of $\varepsilon$, since both sides of these variational equations are of the same order (viz., $\varepsilon$ ), because of the assumptions made on the applied forces.

Equivalently, the field $\boldsymbol{\zeta}$ satisfies the following unconstrained minimization problem:

$$
\boldsymbol{\zeta} \in \boldsymbol{V}_{M}(\omega) \text { and } j_{M}^{\varepsilon}(\boldsymbol{\zeta})=\inf _{\boldsymbol{\eta} \in \boldsymbol{V}_{M}(\omega)} j_{M}^{\varepsilon}(\boldsymbol{\eta})
$$

where

$$
j_{M}^{\varepsilon}(\boldsymbol{\eta}):=\frac{1}{2} \int_{\omega} \varepsilon a^{\alpha \beta \sigma \tau} \gamma_{\sigma \tau}(\boldsymbol{\eta}) \gamma_{\alpha \beta}(\boldsymbol{\eta}) \sqrt{a} d y-\int_{\omega} p^{i, \varepsilon} \eta_{i} \sqrt{a} d y .
$$

Finally, Ciarlet \& Lods [30] studied all the "remaining" cases where $\boldsymbol{V}_{F}(\omega)=$ $\{\mathbf{0}\}$, e.g., when $S$ is elliptic but length $\gamma_{0}<$ length $\gamma$, or when $S$ is for instance a portion of a hyperboloid of revolution, etc. To give a flavor of their results, consider the important special case where the semi-norm

$$
|\cdot|_{\omega}^{M}: \boldsymbol{\eta}=\eta_{i} \mathbf{a}^{i} \rightarrow|\boldsymbol{\eta}|_{\omega}^{M}=\left\{\sum_{\alpha, \beta}\left\|\gamma_{\alpha \beta}(\boldsymbol{\eta})\right\|_{0, \omega}^{2}\right\}^{1 / 2}
$$

becomes a norm over the space

$$
\boldsymbol{W}(\omega):=\left\{\boldsymbol{\eta} \in \boldsymbol{H}^{1}\left(\omega ; \mathbb{R}^{3}\right) ; \boldsymbol{\eta}=\mathbf{0} \text { on } \gamma_{0}\right\} .
$$

In this case, Ciarlet \& Lods [30] showed that, if the applied body forces are "admissible" in a specific sense (but a bit too technical to be described here), and if their density is again $O(1)$ with respect to $\varepsilon$, then

$$
\frac{1}{2 \varepsilon} \int_{-\varepsilon}^{\varepsilon} \boldsymbol{u}^{\varepsilon} d x_{3}^{\varepsilon} \longrightarrow \boldsymbol{\zeta} \text { in } \boldsymbol{V}_{M}^{\sharp}(\omega) \text { as } \varepsilon \rightarrow 0,
$$

where

$$
\boldsymbol{V}_{M}^{\sharp}(\omega):=\text { completion of } \boldsymbol{W}(\omega) \text { with respect to }|\cdot|_{\omega}^{M} \text {. }
$$

Furthermore, the limit field $\boldsymbol{\zeta} \in \boldsymbol{V}_{M}^{\sharp}(\omega)$ solves "limit" variational equations of the form

$$
\varepsilon B_{M}^{\sharp}\left(\boldsymbol{\zeta}^{\varepsilon}, \boldsymbol{\eta}\right)=L_{M}^{\sharp, \varepsilon}(\boldsymbol{\eta}) \text { for all } \boldsymbol{\eta} \in \boldsymbol{V}_{M}^{\sharp}(\omega),
$$

where $B_{M}^{\sharp}$ is the unique extension to $\boldsymbol{V}_{M}^{\sharp}(\omega)$ of the bilinear form $B_{M}$ defined by

$$
B_{M}(\boldsymbol{\zeta}, \boldsymbol{\eta}):=\frac{1}{2} \int_{\omega} a^{\alpha \beta \sigma \tau} \gamma_{\sigma \tau}(\boldsymbol{\zeta}) \gamma_{\alpha \beta}(\boldsymbol{\eta}) \sqrt{a} d y \text { for all } \boldsymbol{\zeta}, \boldsymbol{\eta} \in \boldsymbol{W}(\omega),
$$

i.e., $\varepsilon B_{M}$ is the bilinear form found above for a linearly elastic "membrane shell", and $L_{M}^{\sharp, \varepsilon}: \boldsymbol{V}_{M}^{\sharp}(\omega) \rightarrow \boldsymbol{R}$ is an ad hoc linear form, determined by the behavior as $\varepsilon \rightarrow 0$ of the admissible body forces.

In the "last" remaining case, where $\boldsymbol{V}_{F}(\omega)=\{\mathbf{0}\}$ but $|\cdot|_{\omega}^{M}$ is not a norm over the space $\boldsymbol{W}(\omega)$, a similar convergence result can be established, but only in the 
completion $\dot{\boldsymbol{V}}_{M}^{\sharp}(\omega)$ with respect of $|\cdot|_{\omega}^{M}$ of the quotient space $\boldsymbol{W}(\omega) / \boldsymbol{W}_{0}(\omega)$, where $\boldsymbol{W}_{0}(\omega)=\left\{\boldsymbol{\eta} \in \boldsymbol{W}(\omega) ; \gamma_{\alpha \beta}(\boldsymbol{\eta})=0\right.$ in $\left.\omega\right\}$.

Either one of the above variational problems corresponding to the "remaining" cases where $\boldsymbol{V}_{F}=\{\boldsymbol{0}\}$ constitute the equations of a linearly elastic "generalized" membrane shell, whose two-dimensional equations are therefore justified.

The proofs of the above convergence results are long and technically difficult. Suffice it to say here that they crucially hinge on the Korn inequality "with boundary conditions" (Theorem 2.9-3) and on the Korn inequality "on an elliptic surface" (end of Section 2.9).

Combining these convergences with earlier results of Destuynder [45] and SanchezPalencia [75, 76, 78] (see also Sanchez-Hubert \& Sanchez-Palencia [79]), Ciarlet \& Lods $[28,29]$ have also justified as follows the linear Koiter shell equations studied in Sections 2.8 to 2.10, again in all possible cases.

Let $\boldsymbol{\zeta}^{\varepsilon}$ denote for each $\varepsilon>0$ the unique solution (Theorem 2.10-2) to the linear Koiter shell equations, viz., the vector field that satisfies

$$
\begin{gathered}
\boldsymbol{\zeta}^{\varepsilon} \in \boldsymbol{V}(\omega)=\left\{\boldsymbol{\eta}=\eta_{i} \mathbf{a}^{i} ; \eta_{\alpha} \in H^{1}(\omega), \eta_{3} \in H^{2}(\omega) ; \eta_{i}=\partial_{\nu} \eta_{3}=0 \text { on } \gamma_{0}\right\}, \\
\int_{\omega}\left\{\varepsilon a^{\alpha \beta \sigma \tau} \gamma_{\sigma \tau}\left(\boldsymbol{\zeta}^{\varepsilon}\right) \gamma_{\alpha \beta}(\boldsymbol{\eta})+\frac{\varepsilon^{3}}{3} a^{\alpha \beta \sigma \tau} \rho_{\sigma \tau}\left(\boldsymbol{\zeta}^{\varepsilon}\right) \rho_{\alpha \beta}(\boldsymbol{\eta})\right\} \sqrt{a} d y \\
=\int_{\omega} p^{i, \varepsilon} \eta_{i} \sqrt{a} d y \text { for all } \boldsymbol{\eta}=\eta_{i} \mathbf{a}^{i} \in \boldsymbol{V}(\omega),
\end{gathered}
$$

or equivalently, the unique solution to the minimization problem

$$
\boldsymbol{\zeta}^{\varepsilon} \in \boldsymbol{V}(\omega) \text { and } j\left(\boldsymbol{\zeta}^{\varepsilon}\right)=\inf _{\boldsymbol{\eta} \in \boldsymbol{V}(\omega)} j(\boldsymbol{\eta})
$$

where

$$
\begin{aligned}
j(\boldsymbol{\eta})= & \frac{1}{2} \int_{\omega}\left\{\varepsilon a^{\alpha \beta \sigma \tau} \gamma_{\sigma \tau}(\boldsymbol{\eta}) \gamma_{\alpha \beta}(\boldsymbol{\eta})+\frac{\varepsilon^{3}}{3} a^{\alpha \beta \sigma \tau} \rho_{\sigma \tau}(\boldsymbol{\eta}) \rho_{\alpha \beta}(\boldsymbol{\eta})\right\} \sqrt{a} d y \\
& -\int_{\omega} p^{i, \varepsilon} \eta_{i} \sqrt{a} d y .
\end{aligned}
$$

Observe in passing that, for a linearly elastic shell, the stored energy function found in Koiter's energy, viz.,

$$
\boldsymbol{\eta} \longrightarrow\left\{\frac{\varepsilon}{2} a^{\alpha \beta \sigma \tau} \gamma_{\sigma \tau}(\boldsymbol{\eta}) \gamma_{\alpha \beta}(\boldsymbol{\eta})+\frac{\varepsilon^{3}}{6} a^{\alpha \beta \sigma \tau} \rho_{\sigma \tau}(\boldsymbol{\eta}) \rho_{\alpha \beta}(\boldsymbol{\eta})\right\}
$$

is thus exactly the sum of the stored energy function of a linearly elastic "membrane shell" and of that of a linearly elastic "flexural shell".

Then, for each category of linearly elastic shells (membrane, generalized membrane, or flexural), the vector fields $\boldsymbol{\zeta}^{\varepsilon}$ and $\frac{1}{2 \varepsilon} \int_{-\varepsilon}^{\varepsilon} \boldsymbol{u}^{\varepsilon} d x_{3}^{\varepsilon}$, where $\boldsymbol{u}^{\varepsilon}$ denotes the solution of the three-dimensional problem, have exactly the same asymptotic behavior as $\varepsilon \rightarrow 0$, in precisely the same function spaces that were found in the asymptotic analysis of the three-dimensional solution.

It is all the more remarkable that Koiter's equations can be fully justified for all types of shells, since it is clear that Koiter's equations cannot be recovered as the outcome of an asymptotic analysis of the three-dimensional equations, the two-dimensional equations of linearly elastic, membrane, generalized membrane, or flexural, shells exhausting all such possible outcomes! 
So, even though Koiter's linear model is not a limit model, it is in a sense the "best" two-dimensional one for linearly elastic shells!

One can thus only marvel at the insight that led W.T. Koiter to conceive the "right" equations, whose versatility is indeed remarkable, out of purely mechanical and geometrical intuitions!

We refer to Ciarlet [20] for a detailed analysis of the asymptotic analysis of linearly elastic shells, for a detailed description and analysis of other linear shell models, such as those of Naghdi, Budiansky and Sanders, Novozilov, etc., and for an extensive list of references.

\subsection{The NOnLinear Koiter Shell MOdel}

pagestylemyheadings

In this section, we begin our study of the equations proposed by W.T. Koiter for modeling thin elastic shells. These equations are "two-dimensional", in the sense that they are expressed in terms of two curvilinear coordinates used for defining the middle surface of the shell.

To begin with, we describe the nonlinear Koiter shell equations, so named after Koiter [59], and since then a two-dimensional nonlinear model of choice in computational mechanics.

Given an arbitrary displacement field $\boldsymbol{\eta}:=\eta_{i} \mathbf{a}^{i}: \bar{\omega} \rightarrow \mathbb{R}^{3}$ of the surface $S$ with smooth enough components $\eta_{i}: \bar{\omega} \rightarrow \mathbb{R}$, let

$$
a_{\alpha \beta}(\boldsymbol{\eta}):=\mathbf{a}_{\alpha}(\boldsymbol{\eta}) \cdot \mathbf{a}_{\beta}(\boldsymbol{\eta}), \text { where } \mathbf{a}_{\alpha}(\boldsymbol{\eta}):=\partial_{\alpha}(\boldsymbol{\theta}+\boldsymbol{\eta}),
$$

denote the covariant components of the first fundamental form of the deformed surface $(\boldsymbol{\theta}+\boldsymbol{\eta})(\bar{\omega})$. Then the functions

$$
G_{\alpha \beta}(\boldsymbol{\eta}):=\frac{1}{2}\left(a_{\alpha \beta}(\boldsymbol{\eta})-a_{\alpha \beta}\right)
$$

denote the covariant components of the change of metric tensor associated with the displacement field $\boldsymbol{\eta}=\eta_{i} \mathbf{a}^{i}$ of $S$.

If the two vectors $\mathbf{a}_{\alpha}(\boldsymbol{\eta})$ are linearly independent at all points of $\omega$, let

$$
b_{\alpha \beta}(\boldsymbol{\eta}):=\frac{1}{\sqrt{a(\boldsymbol{\eta})}} \partial_{\alpha \beta}(\boldsymbol{\theta}+\boldsymbol{\eta}) \cdot\left\{\mathbf{a}_{1}(\boldsymbol{\eta}) \wedge \mathbf{a}_{2}(\boldsymbol{\eta})\right\},
$$

where

$$
a(\boldsymbol{\eta}):=\operatorname{det}\left(a_{\alpha \beta}(\boldsymbol{\eta})\right),
$$

denote the covariant components of the second fundamental form of the deformed surface $(\boldsymbol{\theta}+\boldsymbol{\eta})(\bar{\omega})$. Then the functions

$$
R_{\alpha \beta}(\boldsymbol{\eta}):=b_{\alpha \beta}(\boldsymbol{\eta})-b_{\alpha \beta}
$$

denote the covariant components of the change of curvature tensor field associated with the displacement field $\boldsymbol{\eta}=\eta_{i} \mathbf{a}^{i}$ of $S$. Note that $\sqrt{a(\boldsymbol{\eta})}=\mid \mathbf{a}_{1}(\boldsymbol{\eta}) \wedge$ $\mathbf{a}_{2}(\boldsymbol{\eta}) \mid$.

Note that both surfaces $\boldsymbol{\theta}(\bar{\omega})$ and $(\boldsymbol{\theta}+\boldsymbol{\eta})(\bar{\omega})$ are equipped with the same curvilinear coordinates $y_{1}, y_{2}$.

As a point of departure, consider an elastic shell made of a St Venant-Kichhoff material modeled as a three-dimensional problem (Section 2.3). The nonlinear two-dimensional equations proposed by Koiter [59] for modeling such an elastic shell are then derived from those of nonlinear three-dimensional elasticity on the basis of two a priori assumptions: One assumption, of a geometrical nature, is 
the Kirchhoff-Love assumption. It asserts that any point situated on a normal to the middle surface remains on the normal to the deformed middle surface after the deformation has taken place and that, in addition, the distance between such a point and the middle surface remains constant. The other assumption, of a mechanical nature, asserts that the state of stress inside the shell is planar and parallel to the middle surface (this second assumption is itself based on delicate a priori estimates due to John $[56,57])$.

Taking these a priori assumptions into account, W.T. Koiter then reached the conclusion that the displacement field $\boldsymbol{\zeta}^{\varepsilon}=\zeta_{i}^{\varepsilon} \mathbf{a}^{i}$ of the middle surface $S:=\boldsymbol{\theta}(\bar{\omega})$ of the shell, where the functions $\zeta_{i}^{\varepsilon}$ are unknowns, should be a stationary point, in particular a minimizer, over a set of smooth enough vector fields $\boldsymbol{\eta}=\eta_{i} \mathbf{a}^{i}: \bar{\omega} \rightarrow \mathbb{R}^{3}$ satisfying ad hoc boundary conditions on $\gamma_{0}$, of the functional $j$ defined by (cf. Koiter [59], eqs. (4.2), (8.1), and (8.3)):

$$
\begin{aligned}
j(\boldsymbol{\eta})= & \frac{1}{2} \int_{\omega}\left\{\varepsilon a^{\alpha \beta \sigma \tau} G_{\sigma \tau}(\boldsymbol{\eta}) G_{\alpha \beta}(\boldsymbol{\eta})+\frac{\varepsilon^{3}}{3} a^{\alpha \beta \sigma \tau} R_{\sigma \tau}(\boldsymbol{\eta}) R_{\alpha \beta}(\boldsymbol{\eta})\right\} \sqrt{a} d y \\
& -\int_{\omega} p^{i, \varepsilon} \eta_{i} \sqrt{a} d y,
\end{aligned}
$$

where the functions $a^{\alpha \beta \sigma \tau}$ and $p^{i, \varepsilon} \in L^{2}(\omega)$ are the same as in Section 2.6, i.e., they are defined by

$$
\begin{aligned}
a^{\alpha \beta \sigma \tau} & :=\frac{4 \lambda \mu}{\lambda+2 \mu} a^{\alpha \beta} a^{\sigma \tau}+2 \mu\left(a^{\alpha \sigma} a^{\beta \tau}+a^{\alpha \tau} a^{\beta \sigma}\right) \\
p^{i, \varepsilon} & :=\int_{-\varepsilon}^{\varepsilon} f^{i, \varepsilon} d x_{3} .
\end{aligned}
$$

The above functional $j$ is called Koiter's energy for a nonlinear elastic shell.

The stored energy function $w_{K}$ found in Koiter's energy $j$ is thus defined by

$$
w_{K}(\boldsymbol{\eta})=\frac{\varepsilon}{2} a^{\alpha \beta \sigma \tau} G_{\sigma \tau}(\boldsymbol{\eta}) G_{\alpha \beta}(\boldsymbol{\eta})+\frac{\varepsilon^{3}}{6} a^{\alpha \beta \sigma \tau} R_{\sigma \tau}(\boldsymbol{\eta}) R_{\alpha \beta}(\boldsymbol{\eta})
$$

for ad hoc vector fields $\boldsymbol{\eta}=\eta_{i} \mathbf{a}^{i}$. This expression is the sum of the "membrane" part

$$
w_{M}(\boldsymbol{\eta})=\frac{\varepsilon}{2} a^{\alpha \beta \sigma \tau} G_{\sigma \tau}(\boldsymbol{\eta}) G_{\alpha \beta}(\boldsymbol{\eta})
$$

and of the "flexural" part

$$
w_{F}(\boldsymbol{\eta})=\frac{\varepsilon^{3}}{6} a^{\alpha \beta \sigma \tau} R_{\sigma \tau}(\boldsymbol{\eta}) R_{\alpha \beta}(\boldsymbol{\eta}) .
$$

Another closely related set of nonlinear shell equations "of Koiter's type" has been proposed by Ciarlet [21]. In these equations, the denominator $\sqrt{a(\boldsymbol{\eta})}$ that appears in the functions $R_{\alpha \beta}(\boldsymbol{\eta})=b_{\alpha \beta}(\boldsymbol{\eta})-b_{\alpha \beta}$ is simply replaced by $\sqrt{a}$, thereby avoiding the possibility of a vanishing denominator in the expression $w_{K}(\boldsymbol{\eta})$. Then Ciarlet \& Roquefort [34] have shown that the leading term of a formal asymptotic expansion of a solution to this two-dimensional model, with the thickness $2 \varepsilon$ as the "small" parameter, coincides with that found by a formal asymptotic analysis of the three-dimensional equations. This result thus raises hopes that a rigorous justification, by means of $\Gamma$-convergence theory, of a nonlinear shell model of Koiter's type might be possible. 


\subsection{The LineAR KoITER SHELl MODEL}

Consider the Koiter energy $j$ for a nonlinearly elastic shell, defined by (cf. Section 2.7)

$$
\begin{aligned}
j(\boldsymbol{\eta})= & \frac{1}{2} \int_{\omega}\left\{\varepsilon a^{\alpha \beta \sigma \tau} G_{\sigma \tau}(\boldsymbol{\eta}) G_{\alpha \beta}(\boldsymbol{\eta})+\frac{\varepsilon^{3}}{3} a^{\alpha \beta \sigma \tau} R_{\sigma \tau}(\boldsymbol{\eta}) R_{\alpha \beta}(\boldsymbol{\eta})\right\} \sqrt{a} d y \\
& -\int_{\omega} p^{i, \varepsilon} \eta_{i} \sqrt{a} d y,
\end{aligned}
$$

for smooth enough vector fields $\boldsymbol{\eta}=\eta_{i} \mathbf{a}^{i}: \bar{\omega} \rightarrow \mathbb{R}^{3}$. One of its virtues is that the integrands of the first two integrals are quadratic expressions in terms of the covariant components $G_{\alpha \beta}(\boldsymbol{\eta})$ and $R_{\alpha \beta}(\boldsymbol{\eta})$ of the change of metric, and change of curvature, tensors associated with a displacement field $\boldsymbol{\eta}=\eta_{i} \mathbf{a}^{i}$ of the middle surface $S=\boldsymbol{\theta}(\bar{\omega})$ of the shell. In order to obtain the energy corresponding to the linear equations of Koiter [60], which we are about to describe, it suffices, "by definition", to replace the covariant components

$$
G_{\alpha \beta}(\boldsymbol{\eta})=\frac{1}{2}\left(a_{\alpha \beta}(\boldsymbol{\eta})-a_{\alpha \beta}\right) \text { and } R_{\alpha \beta}(\boldsymbol{\eta})=b_{\alpha \beta}(\boldsymbol{\eta})-b_{\alpha \beta},
$$

of these tensors by their linear parts with respect to $\boldsymbol{\eta}$, respectively denoted $\gamma_{\alpha \beta}(\boldsymbol{\eta})$ and $\rho_{\alpha \beta}(\boldsymbol{\eta})$ below. Accordingly, our first task consists in finding explicit expressions of such linearized tensors. To begin with, we compute the components $\gamma_{\alpha \beta}(\boldsymbol{\eta})$.

Theorem 2.8-1. Let $\omega$ be a domain in $\mathbb{R}^{2}$ and let $\boldsymbol{\theta} \in \mathcal{C}^{2}\left(\bar{\omega} ; \mathbb{R}^{3}\right)$ be an immersion. Given a displacement field $\boldsymbol{\eta}:=\eta_{i} \mathbf{a}^{i}$ of the surface $S=\boldsymbol{\theta}(\bar{\omega})$ with smooth enough covariant components $\eta_{i}: \bar{\omega} \rightarrow \mathbb{R}$, let the function $\gamma_{\alpha \beta}(\boldsymbol{\eta}): \bar{\omega} \rightarrow \mathbb{R}$ be defined by

$$
\gamma_{\alpha \beta}(\boldsymbol{\eta}):=\frac{1}{2}\left[a_{\alpha \beta}(\boldsymbol{\eta})-a_{\alpha \beta}\right]^{\text {lin }},
$$

where $a_{\alpha \beta}$ and $a_{\alpha \beta}(\boldsymbol{\eta})$ are the covariant components of the first fundamental form of the surfaces $\boldsymbol{\theta}(\bar{\omega})$ and $(\boldsymbol{\theta}+\boldsymbol{\eta})(\bar{\omega})$, and $[\cdots]^{\text {lin }}$ denotes the linear part with respect to $\boldsymbol{\eta}$ in the expression $[\cdots]$. Then

$$
\begin{aligned}
\gamma_{\alpha \beta}(\boldsymbol{\eta}) & =\frac{1}{2}\left(\partial_{\beta} \boldsymbol{\eta} \cdot \mathbf{a}_{\alpha}+\partial_{\alpha} \boldsymbol{\eta} \cdot \mathbf{a}_{\beta}\right)=\gamma_{\beta \alpha}(\boldsymbol{\eta}) \\
& =\frac{1}{2}\left(\eta_{\alpha \mid \beta}+\eta_{\beta \mid \alpha}\right)-b_{\alpha \beta} \eta_{3} \\
& =\frac{1}{2}\left(\partial_{\beta} \eta_{\alpha}+\partial_{\alpha} \eta_{\beta}\right)-\Gamma_{\alpha \beta}^{\sigma} \eta_{\sigma}-b_{\alpha \beta} \eta_{3},
\end{aligned}
$$

where the covariant derivatives $\eta_{\alpha \mid \beta}$ are defined by $\eta_{\alpha \mid \beta}=\partial_{\beta} \eta_{\alpha}-\Gamma_{\alpha \beta}^{\sigma} \eta_{\sigma}$. In particular then,

$$
\eta_{\alpha} \in H^{1}(\omega) \text { and } \eta_{3} \in L^{2}(\omega) \Rightarrow \gamma_{\alpha \beta}(\boldsymbol{\eta}) \in L^{2}(\omega) .
$$

Proof. The covariant components $a_{\alpha \beta}(\boldsymbol{\eta})$ of the metric tensor of the surface $(\boldsymbol{\theta}+$ $\boldsymbol{\eta})(\bar{\omega})$ are by definition given by

$$
a_{\alpha \beta}(\boldsymbol{\eta})=\partial_{\alpha}(\boldsymbol{\theta}+\boldsymbol{\eta}) \cdot \partial_{\beta}(\boldsymbol{\theta}+\boldsymbol{\eta}) .
$$

The relations

$$
\partial_{\alpha}(\boldsymbol{\theta}+\boldsymbol{\eta})=\mathbf{a}_{\alpha}+\partial_{\alpha} \boldsymbol{\eta}
$$


then show that

$$
\begin{aligned}
a_{\alpha \beta}(\boldsymbol{\eta}) & =\left(\mathbf{a}_{\alpha}+\partial_{\alpha} \boldsymbol{\eta}\right) \cdot\left(\mathbf{a}_{\beta}+\partial_{\beta} \boldsymbol{\eta}\right) \\
& =a_{\alpha \beta}+\partial_{\beta} \boldsymbol{\eta} \cdot \mathbf{a}_{\alpha}+\partial_{\alpha} \boldsymbol{\eta} \cdot \mathbf{a}_{\beta}+\partial_{\alpha} \boldsymbol{\eta} \cdot \partial_{\beta} \boldsymbol{\eta},
\end{aligned}
$$

hence that

$$
\gamma_{\alpha \beta}(\boldsymbol{\eta})=\frac{1}{2}\left[a_{\alpha \beta}(\boldsymbol{\eta})-a_{\alpha \beta}\right]^{\text {lin }}=\frac{1}{2}\left(\partial_{\beta} \boldsymbol{\eta} \cdot \mathbf{a}_{\alpha}+\partial_{\alpha} \boldsymbol{\eta} \cdot \mathbf{a}_{\beta}\right) .
$$

The other expressions of $\gamma_{\alpha \beta}(\boldsymbol{\eta})$ immediately follow from the relation

$$
\partial_{\alpha} \boldsymbol{\eta}=\partial_{\alpha}\left(\eta_{i} \mathbf{a}^{i}\right)=\left(\partial_{\alpha} \eta_{\sigma}-\Gamma_{\alpha \sigma}^{\tau} \eta_{\tau}-b_{\alpha \sigma} \eta_{3}\right) \mathbf{a}^{\sigma}+\left(\partial_{\alpha} \eta_{3}+b_{\alpha}^{\tau} \eta_{\tau}\right) \mathbf{a}^{3},
$$

itself a consequence of the Gauss and Weingarten equations (see Section 2.1)

$$
\begin{aligned}
& \partial_{\alpha} \mathbf{a}^{\tau}=-\Gamma_{\alpha \sigma}^{\tau} \mathbf{a}^{\sigma}+b_{\alpha}^{\tau} \mathbf{a}^{3}, \\
& \partial_{\alpha} \mathbf{a}^{3}=-b_{\alpha \sigma} \mathbf{a}^{\sigma} .
\end{aligned}
$$

The functions $\gamma_{\alpha \beta}(\boldsymbol{\eta})$ are called the covariant components of the linearized change of metric tensor associated with a displacement $\boldsymbol{\eta}=\eta_{i} \mathbf{a}^{i}$ of the surface $S$.

We next compute the components $\rho_{\alpha \beta}(\boldsymbol{\eta})$.

Theorem 2.8-2. Let $\omega$ be a domain in $\mathbb{R}^{2}$ and let $\boldsymbol{\theta} \in \mathcal{C}^{3}\left(\bar{\omega} ; \mathbb{R}^{3}\right)$ be an immersion. Given a displacement field $\boldsymbol{\eta}:=\eta_{i} \mathbf{a}^{i}$ of the surface $S=\boldsymbol{\theta}(\bar{\omega})$ with smooth enough and "small enough" covariant components $\eta_{i}: \bar{\omega} \rightarrow \mathbb{R}$, let the functions $\rho_{\alpha \beta}(\boldsymbol{\eta})$ : $\bar{\omega} \rightarrow \mathbb{R}$ be defined by

$$
\rho_{\alpha \beta}(\boldsymbol{\eta}):=\left[b_{\alpha \beta}(\boldsymbol{\eta})-b_{\alpha \beta}\right]^{\text {lin }},
$$

where $b_{\alpha \beta}$ and $b_{\alpha \beta}(\boldsymbol{\eta})$ are the covariant components of the second fundamental form of the surfaces $\boldsymbol{\theta}(\bar{\omega})$ and $(\boldsymbol{\theta}+\boldsymbol{\eta})(\bar{\omega})$, and $[\cdots]^{\text {lin }}$ denotes the linear part with respect to $\boldsymbol{\eta}$ in the expression $[\cdots]$. Then

$$
\begin{aligned}
\rho_{\alpha \beta}(\boldsymbol{\eta})= & \left(\partial_{\alpha \beta} \boldsymbol{\eta}-\Gamma_{\alpha \beta}^{\sigma} \partial_{\sigma} \boldsymbol{\eta}\right) \cdot \mathbf{a}_{3}=\rho_{\beta \alpha}(\boldsymbol{\eta}) \\
= & \eta_{3 \mid \alpha \beta}-b_{\alpha}^{\sigma} b_{\sigma \beta} \eta_{3}+b_{\alpha}^{\sigma} \eta_{\sigma \mid \beta}+b_{\beta}^{\tau} \eta_{\tau \mid \alpha}+\left.b_{\beta}^{\tau}\right|_{\alpha} \eta_{\tau} \\
= & \partial_{\alpha \beta} \eta_{3}-\Gamma_{\alpha \beta}^{\sigma} \partial_{\sigma} \eta_{3}-b_{\alpha}^{\sigma} b_{\sigma \beta} \eta_{3} \\
& +b_{\alpha}^{\sigma}\left(\partial_{\beta} \eta_{\sigma}-\Gamma_{\beta \sigma}^{\tau} \eta_{\tau}\right)+b_{\beta}^{\tau}\left(\partial_{\alpha} \eta_{\tau}-\Gamma_{\alpha \tau}^{\sigma} \eta_{\sigma}\right) \\
& +\left(\partial_{\alpha} b_{\beta}^{\tau}+\Gamma_{\alpha \sigma}^{\tau} b_{\beta}^{\sigma}-\Gamma_{\alpha \beta}^{\sigma} b_{\sigma}^{\tau}\right) \eta_{\tau},
\end{aligned}
$$

where the covariant derivatives $\eta_{\alpha \mid \beta}, \eta_{3 \mid \alpha \beta}$, and $\left.b_{\beta}^{\tau}\right|_{\alpha}$ are defined by

$$
\begin{aligned}
\eta_{\alpha \mid \beta} & :=\partial_{\beta} \eta_{\alpha}-\Gamma_{\alpha \beta}^{\sigma} \eta_{\sigma}, \\
\eta_{3 \mid \alpha \beta} & :=\partial_{\alpha \beta} \eta_{3}-\Gamma_{\alpha \beta}^{\sigma} \partial_{\sigma} \eta_{3}, \\
\left.b_{\beta}^{\tau}\right|_{\alpha} & :=\partial_{\alpha} b_{\beta}^{\tau}+\Gamma_{\alpha \sigma}^{\tau} b_{\beta}^{\sigma}-\Gamma_{\alpha \beta}^{\sigma} b_{\sigma}^{\tau} .
\end{aligned}
$$

In particular then,

$$
\eta_{\alpha} \in H^{1}(\omega) \text { and } \eta_{3} \in H^{2}(\omega) \Rightarrow \rho_{\alpha \beta}(\boldsymbol{\eta}) \in L^{2}(\omega) .
$$

The functions $\left.b_{\beta}^{\tau}\right|_{\alpha}$ satisfy the symmetry relations

$$
\left.b_{\beta}^{\tau}\right|_{\alpha}=\left.b_{\alpha}^{\tau}\right|_{\beta} .
$$


Proof. For convenience, the proof is divided into five parts. In parts (i) and (ii), we establish elementary relations satisfied by the vectors $\mathbf{a}_{i}$ and $\mathbf{a}^{i}$ of the covariant and contravariant bases along $S$.

(i) The two vectors $\mathbf{a}_{\alpha}=\partial_{\alpha} \boldsymbol{\theta}$ satisfy $\left|\mathbf{a}_{1} \wedge \mathbf{a}_{2}\right|=\sqrt{a}$, where $a=\operatorname{det}\left(a_{\alpha \beta}\right)$.

Let $\boldsymbol{A}$ denote the matrix of order three with $\mathbf{a}_{1}, \mathbf{a}_{2}, \mathbf{a}_{3}$ as its column vectors. Consequently,

$$
\operatorname{det} \boldsymbol{A}=\left(\mathbf{a}_{1} \wedge \mathbf{a}_{2}\right) \cdot \mathbf{a}_{3}=\left(\mathbf{a}_{1} \wedge \mathbf{a}_{2}\right) \cdot \frac{\mathbf{a}_{1} \wedge \mathbf{a}_{2}}{\left|\mathbf{a}_{1} \wedge \mathbf{a}_{2}\right|}=\left|\mathbf{a}_{1} \wedge \mathbf{a}_{2}\right| .
$$

Besides,

$$
(\operatorname{det} \boldsymbol{A})^{2}=\operatorname{det}\left(\boldsymbol{A}^{T} \boldsymbol{A}\right)=\operatorname{det}\left(a_{\alpha \beta}\right)=a,
$$

since $\mathbf{a}_{\alpha} \cdot \mathbf{a}_{\beta}=a_{\alpha \beta}$ and $\mathbf{a}_{\alpha} \cdot \mathbf{a}_{3}=\delta_{\alpha 3}$. Hence $\left|\mathbf{a}_{1} \wedge \mathbf{a}_{2}\right|=\sqrt{a}$.

(ii) The vector fields $\mathbf{a}_{i}$ and $\mathbf{a}^{\alpha}$ are related by $\mathbf{a}_{1} \wedge \mathbf{a}_{3}=-\sqrt{a} \mathbf{a}^{2}$ and $\mathbf{a}_{3} \wedge \mathbf{a}_{2}=$ $-\sqrt{a} \mathbf{a}^{1}$.

To prove that two vector fields $\boldsymbol{c}$ and $\boldsymbol{d}$ coincide, it suffices to prove that $\boldsymbol{c} \cdot \mathbf{a}_{i}=$ $\boldsymbol{d} \cdot \mathbf{a}_{i}$ for $i \in\{1,2,3\}$. In the present case,

$$
\begin{aligned}
& \left(\mathbf{a}_{1} \wedge \mathbf{a}_{3}\right) \cdot \mathbf{a}_{1}=0 \text { and }\left(\mathbf{a}_{1} \wedge \mathbf{a}_{3}\right) \cdot \mathbf{a}_{3}=0, \\
& \left(\mathbf{a}_{1} \wedge \mathbf{a}_{3}\right) \cdot \mathbf{a}_{2}=-\left(\mathbf{a}_{1} \wedge \mathbf{a}_{2}\right) \cdot \mathbf{a}_{3}=-\sqrt{a},
\end{aligned}
$$

since $\sqrt{a} \mathbf{a}_{3}=\mathbf{a}_{1} \wedge \mathbf{a}_{2}$ by (i), on the one hand; on the other hand,

$$
-\sqrt{a} \mathbf{a}^{2} \cdot \mathbf{a}_{1}=-\sqrt{a} \mathbf{a}^{2} \cdot \mathbf{a}_{3}=0 \text { and }-\sqrt{a} \mathbf{a}^{2} \cdot \mathbf{a}_{2}=-\sqrt{a},
$$

since $\mathbf{a}^{i} \cdot \mathbf{a}_{j}=\delta_{j}^{i}$. Hence $\mathbf{a}_{1} \wedge \mathbf{a}_{3}=-\sqrt{a} \mathbf{a}^{2}$. The other relation is similarly established.

(iii) The covariant components $b_{\alpha \beta}(\boldsymbol{\eta})$ satisfy

$$
b_{\alpha \beta}(\boldsymbol{\eta})=b_{\alpha \beta}+\left(\partial_{\alpha \beta} \boldsymbol{\eta}-\Gamma_{\alpha \beta}^{\sigma} \partial_{\sigma} \boldsymbol{\eta}\right) \cdot \mathbf{a}_{3}+\text { h.o.t. }
$$

where "h.o.t." stands for "higher-order terms", i.e., terms of order higher than linear with respect to $\boldsymbol{\eta}$. Consequently,

$$
\rho_{\alpha \beta}(\boldsymbol{\eta}):=\left[b_{\alpha \beta}(\boldsymbol{\eta})-b_{\alpha \beta}\right]^{\text {lin }}=\left(\partial_{\alpha \beta} \boldsymbol{\eta}-\Gamma_{\alpha \beta}^{\sigma} \partial_{\sigma} \boldsymbol{\eta}\right) \cdot \mathbf{a}_{3}=\rho_{\beta \alpha}(\boldsymbol{\eta}) .
$$

Since the vectors $\mathbf{a}_{\alpha}=\partial_{\alpha} \boldsymbol{\theta}$ are linearly independent in $\bar{\omega}$ and the fields $\boldsymbol{\eta}=$ $\eta_{i} \mathbf{a}_{i}$ are smooth enough by assumption, the vectors $\partial_{\alpha}(\boldsymbol{\theta}+\boldsymbol{\eta})$ are also linearly independent in $\bar{\omega}$ provided the fields $\boldsymbol{\eta}$ are "small enough", e.g., with respect to the norm of the space $\mathcal{C}^{1}\left(\bar{\omega} ; \mathbb{R}^{3}\right)$. The following computations are therefore licit as they apply to a linearization around $\boldsymbol{\eta}=\mathbf{0}$.

Let

$$
\mathbf{a}_{\alpha}(\boldsymbol{\eta}):=\partial_{\alpha}(\boldsymbol{\theta}+\boldsymbol{\eta})=\mathbf{a}_{\alpha}+\partial_{\alpha} \boldsymbol{\eta} \text { and } \mathbf{a}_{3}(\boldsymbol{\eta}):=\frac{\mathbf{a}_{1}(\boldsymbol{\eta}) \wedge \mathbf{a}_{2}(\boldsymbol{\eta})}{\sqrt{a(\boldsymbol{\eta})}}
$$

where

$$
a(\boldsymbol{\eta}):=\operatorname{det}\left(a_{\alpha \beta}(\boldsymbol{\eta})\right) \text { and } a_{\alpha \beta}(\boldsymbol{\eta}):=a_{\alpha}(\boldsymbol{\eta}) \cdot \mathbf{a}_{\beta}(\eta) \text {. }
$$


Then

$$
\begin{aligned}
& b_{\alpha \beta}(\boldsymbol{\eta})=\partial_{\alpha} \mathbf{a}_{\beta}(\boldsymbol{\eta}) \cdot \mathbf{a}_{3}(\boldsymbol{\eta}) \\
& =\frac{1}{\sqrt{a(\boldsymbol{\eta})}}\left(\partial_{\alpha} \mathbf{a}_{\beta}+\partial_{\alpha \beta} \boldsymbol{\eta}\right) \cdot\left(\mathbf{a}_{1} \wedge \mathbf{a}_{2}+\mathbf{a}_{1} \wedge \partial_{2} \boldsymbol{\eta}+\partial_{1} \boldsymbol{\eta} \wedge \mathbf{a}_{2}+\text { h.o.t. }\right) \\
& =\frac{1}{\sqrt{a(\boldsymbol{\eta})}}\left\{\sqrt{a}\left(b_{\alpha \beta}+\partial_{\alpha \beta} \boldsymbol{\eta} \cdot \mathbf{a}_{3}\right)\right\} \\
& \quad+\frac{1}{\sqrt{a(\boldsymbol{\eta})}}\left\{\left(\Gamma_{\alpha \beta}^{\sigma} \mathbf{a}_{\sigma}+b_{\alpha \beta} \mathbf{a}_{3}\right) \cdot\left(\mathbf{a}_{1} \wedge \partial_{2} \boldsymbol{\eta}+\partial_{1} \boldsymbol{\eta} \wedge \mathbf{a}_{2}\right)+\text { h.o.t. }\right\},
\end{aligned}
$$

since $b_{\alpha \beta}=\partial_{\alpha} \mathbf{a}_{\beta} \cdot \mathbf{a}_{3}$ and $\partial_{\alpha} \mathbf{a}_{\beta}=\Gamma_{\alpha \beta}^{\sigma} \mathbf{a}_{\sigma}+b_{\alpha \beta} \mathbf{a}_{3}$ by the formula of Gauss. Next,

$$
\begin{aligned}
& \left(\Gamma_{\alpha \beta}^{\sigma} \mathbf{a}_{\sigma}+b_{\alpha \beta} \mathbf{a}_{3}\right) \cdot\left(\mathbf{a}_{1} \wedge \partial_{2} \boldsymbol{\eta}\right) \\
& =\Gamma_{\alpha \beta}^{2} \mathbf{a}_{2} \cdot\left(\mathbf{a}_{1} \wedge \partial_{2} \boldsymbol{\eta}\right)-b_{\alpha \beta} \partial_{2} \boldsymbol{\eta} \cdot\left(\mathbf{a}_{1} \wedge \mathbf{a}_{3}\right) \\
& =\sqrt{a}\left(-\Gamma_{\alpha \beta}^{2} \partial_{2} \boldsymbol{\eta} \cdot \mathbf{a}_{3}+b_{\alpha \beta} \partial_{2} \boldsymbol{\eta} \cdot \mathbf{a}^{2}\right),
\end{aligned}
$$

since, by (ii), $\mathbf{a}_{2} \cdot\left(\mathbf{a}_{1} \wedge \partial_{2} \boldsymbol{\eta}\right)=-\partial_{2} \boldsymbol{\eta} \cdot\left(\mathbf{a}_{1} \wedge \mathbf{a}_{2}\right)=-\sqrt{a} \partial_{2} \boldsymbol{\eta} \cdot \mathbf{a}_{3}$ and $\mathbf{a}_{1} \wedge \mathbf{a}_{3}=-\sqrt{a} \mathbf{a}^{2}$; likewise,

$$
\left(\Gamma_{\alpha \beta}^{\sigma} \mathbf{a}_{\sigma}+b_{\alpha \beta} \mathbf{a}_{3}\right) \cdot\left(\partial_{1} \boldsymbol{\eta} \wedge \mathbf{a}_{2}\right)=\sqrt{a}\left(-\Gamma_{\alpha \beta}^{1} \partial_{1} \boldsymbol{\eta} \cdot \mathbf{a}_{3}+b_{\alpha \beta} \partial_{1} \boldsymbol{\eta} \cdot \mathbf{a}^{1}\right)
$$

Consequently,

$$
b_{\alpha \beta}(\boldsymbol{\eta})=\sqrt{\frac{a}{a(\boldsymbol{\eta})}}\left\{b_{\alpha \beta}\left(1+\partial_{\sigma} \boldsymbol{\eta} \cdot \mathbf{a}^{\sigma}\right)+\left(\partial_{\alpha \beta} \boldsymbol{\eta}-\Gamma_{\alpha \beta}^{\sigma} \partial_{\sigma} \boldsymbol{\eta}\right) \cdot \mathbf{a}_{3}+\text { h.o.t. }\right\} .
$$

There remains to find the linear term with respect to $\boldsymbol{\eta}$ in the expansion $\frac{1}{\sqrt{a(\boldsymbol{\eta})}}=$ $\frac{1}{\sqrt{a}}(1+\cdots)$. To this end, we note that

$$
\operatorname{det}(\boldsymbol{A}+\boldsymbol{H})=(\operatorname{det} \boldsymbol{A})\left(1+\operatorname{tr}\left(\boldsymbol{A}^{-1} \boldsymbol{H}\right)+o(\boldsymbol{H})\right)
$$

with $\boldsymbol{A}:=\left(a_{\alpha \beta}\right)$ and $\boldsymbol{A}+\boldsymbol{H}:=\left(a_{\alpha \beta}(\boldsymbol{\eta})\right)$. Hence

$$
\boldsymbol{H}=\partial_{\beta} \boldsymbol{\eta} \cdot \mathbf{a}_{\alpha}+\partial_{\alpha} \boldsymbol{\eta} \cdot \mathbf{a}_{\beta}+\text { h.o.t., }
$$

since $\left[a_{\alpha \beta}(\boldsymbol{\eta})-a_{\alpha \beta}\right]^{\operatorname{lin}}=\partial_{\beta} \boldsymbol{\eta} \cdot \mathbf{a}_{\alpha}+\partial_{\alpha} \boldsymbol{\eta} \cdot \mathbf{a}_{\beta}$ (Theorem 2.8-1). Therefore,

$$
a(\boldsymbol{\eta})=\operatorname{det}\left(a_{\alpha \beta}(\boldsymbol{\eta})\right)=\operatorname{det}\left(a_{\alpha \beta}\right)\left(1+2 \partial_{\alpha} \boldsymbol{\eta} \cdot \mathbf{a}^{\alpha}+\text { h.o.t. }\right),
$$

since $\boldsymbol{A}^{-1}=\left(a^{\alpha \beta}\right)$; consequently,

$$
\frac{1}{\sqrt{a(\boldsymbol{\eta})}}=\frac{1}{\sqrt{a}}\left(1-\partial_{\alpha} \boldsymbol{\eta} \cdot \mathbf{a}^{\alpha}+\text { h.o.t. }\right) .
$$

Noting that there are no linear terms with respect to $\boldsymbol{\eta}$ in the product $\left(1-\partial_{\alpha} \boldsymbol{\eta}\right.$. $\left.\mathbf{a}^{\alpha}\right)\left(1+\partial_{\sigma} \boldsymbol{\eta} \cdot \mathbf{a}^{\sigma}\right)$, we find the announced expansion, viz.,

$$
b_{\alpha \beta}(\boldsymbol{\eta})=b_{\alpha \beta}+\left(\partial_{\alpha \beta} \boldsymbol{\eta}-\Gamma_{\alpha \beta}^{\sigma} \partial_{\sigma} \boldsymbol{\eta}\right) \cdot \mathbf{a}_{3}+\text { h.o.t. }
$$

(iv) The components $\rho_{\alpha \beta}(\boldsymbol{\eta})$ can be also written as

$$
\rho_{\alpha \beta}(\boldsymbol{\eta})=\eta_{3 \mid \alpha \beta}-b_{\alpha}^{\sigma} b_{\sigma \beta} \eta_{3}+b_{\alpha}^{\sigma} \eta_{\sigma \mid \beta}+b_{\beta}^{\tau} \eta_{\tau \mid \alpha}+\left.b_{\beta}^{\tau}\right|_{\alpha} \eta_{\tau}
$$

where the functions $\eta_{3 \mid \alpha \beta}$ and $\left.b_{\beta}^{\tau}\right|_{\alpha}$ are defined as in the statement of the theorem. 
The Gauss and Weingarten equations, viz.,

$$
\begin{aligned}
& \partial_{\alpha} \mathbf{a}^{\tau}=-\Gamma_{\alpha \sigma}^{\tau} \mathbf{a}^{\sigma}+b_{\alpha}^{\tau} \mathbf{a}_{3}, \\
& \partial_{\alpha} \mathbf{a}^{3}=-b_{\alpha \sigma} \mathbf{a}^{\sigma}
\end{aligned}
$$

imply that

$$
\partial_{\sigma} \boldsymbol{\eta}=\left(\partial_{\sigma} \eta_{\beta}-\Gamma_{\sigma \beta}^{\tau} \eta_{\tau}-b_{\sigma \beta} \eta_{3}\right) \mathbf{a}^{\beta}+\left(\partial_{\sigma} \eta_{3}+b_{\sigma}^{\tau} \eta_{\tau}\right) \mathbf{a}^{3}
$$

then that

$$
\begin{aligned}
\partial_{\alpha \beta} \boldsymbol{\eta} \cdot \mathbf{a}_{3}= & \partial_{\alpha}\left\{\left(\partial_{\beta} \eta_{\sigma}-\Gamma_{\beta \sigma}^{\tau} \eta_{\tau}-b_{\beta \sigma} \eta_{3}\right) \mathbf{a}^{\sigma}+\left(\partial_{\beta} \eta_{3}+b_{\beta}^{\tau} \eta_{\tau}\right) \mathbf{a}^{3}\right\} \cdot \mathbf{a}_{3} \\
= & \left(\partial_{\beta} \eta_{\sigma}-\Gamma_{\beta \sigma}^{\tau} \eta_{\tau}-b_{\beta \sigma} \eta_{3}\right) \partial_{\alpha} \mathbf{a}^{\sigma} \cdot \mathbf{a}_{3} \\
& +\left(\partial_{\alpha \beta} \eta_{3}+\left(\partial_{\alpha} b_{\beta}^{\tau}\right) \eta_{\tau}+b_{\beta}^{\tau} \partial_{\alpha} \eta_{\tau}\right) \mathbf{a}^{3} \cdot \mathbf{a}_{3}+\left(\partial_{\beta} \eta_{3}+b_{\beta}^{\tau} \eta_{\tau}\right) \partial_{\alpha} \mathbf{a}^{3} \cdot \mathbf{a}_{3} \\
= & b_{\alpha}^{\sigma}\left(\partial_{\beta} \eta_{\sigma}-\Gamma_{\beta \sigma}^{\tau} \eta_{\tau}\right)-b_{\alpha}^{\sigma} b_{\sigma \beta} \eta_{3}+\partial_{\alpha \beta} \eta_{3}+\left(\partial_{\alpha} b_{\beta}^{\tau}\right) \eta_{\tau}+b_{\beta}^{\tau} \partial_{\alpha} \eta_{\tau}
\end{aligned}
$$

since

$$
\begin{gathered}
\partial_{\alpha} \mathbf{a}^{\sigma} \cdot \mathbf{a}_{3}=\left(-\Gamma_{\alpha \tau}^{\sigma} \mathbf{a}^{\tau}+b_{\alpha}^{\sigma} \mathbf{a}^{3}\right) \cdot \mathbf{a}_{3}=b_{\alpha}^{\sigma}, \\
\partial_{\alpha} \mathbf{a}^{3} \cdot \mathbf{a}_{3}=-b_{\alpha \sigma} \mathbf{a}^{\sigma} \cdot \mathbf{a}_{3}=0 .
\end{gathered}
$$

We thus obtain

$$
\begin{aligned}
\rho_{\alpha \beta}(\boldsymbol{\eta})= & \left(\partial_{\alpha \beta} \boldsymbol{\eta}-\Gamma_{\alpha \beta}^{\sigma} \partial_{\sigma} \boldsymbol{\eta}\right) \cdot \mathbf{a}_{3} \\
= & b_{\alpha}^{\sigma}\left(\partial_{\beta} \eta_{\sigma}-\Gamma_{\beta \sigma}^{\tau} \eta_{\tau}\right)-b_{\alpha}^{\sigma} b_{\sigma \beta} \eta_{3}+\partial_{\alpha \beta} \eta_{3}+\left(\partial_{\alpha} b_{\beta}^{\tau}\right) \eta_{\tau}+b_{\beta}^{\tau} \partial_{\alpha} \eta_{\tau} \\
& -\Gamma_{\alpha \beta}^{\sigma}\left(\partial_{\sigma} \eta_{3}+b_{\sigma}^{\tau} \eta_{\tau}\right) .
\end{aligned}
$$

While this relation seemingly involves only the covariant derivatives $\eta_{3 \mid \alpha \beta}$ and $\eta_{\sigma \mid \beta}$, it may be easily rewritten so as to involve in addition the functions $\eta_{\tau \mid \alpha}$ and $b_{\beta \mid \alpha}^{\tau}$. The stratagem simply consists in using the relation $\Gamma_{\alpha \sigma}^{\tau} b_{\beta}^{\sigma} \eta_{\tau}-\Gamma_{\alpha \tau}^{\sigma} b_{\beta}^{\tau} \eta_{\sigma}=0$ ! This gives

$$
\begin{aligned}
\rho_{\alpha \beta}(\boldsymbol{\eta})= & \left(\partial_{\alpha \beta} \eta_{3}-\Gamma_{\alpha \beta}^{\sigma} \partial_{\sigma} \eta_{3}\right)-b_{\alpha}^{\sigma} b_{\sigma \beta} \eta_{3} \\
& +b_{\alpha}^{\sigma}\left(\partial_{\beta} \eta_{\sigma}-\Gamma_{\beta \sigma}^{\tau} \eta_{\tau}\right)+b_{\beta}^{\tau}\left(\partial_{\alpha} \eta_{\tau}-\Gamma_{\alpha \tau}^{\sigma} \eta_{\sigma}\right) \\
& +\left(\partial_{\alpha} b_{\beta}^{\tau}+\Gamma_{\alpha \sigma}^{\tau} b_{\beta}^{\sigma}-\Gamma_{\alpha \beta}^{\sigma} b_{\sigma}^{\tau}\right) \eta_{\tau} .
\end{aligned}
$$

(v) The functions $\left.b_{\beta}^{\tau}\right|_{\alpha}$ are symmetric with respect to the indices $\alpha$ and $\beta$. Again, because of the formulas of Gauss and Weingarten, we can write

$$
\begin{aligned}
\mathbf{0}= & \partial_{\alpha \beta} \mathbf{a}^{\tau}-\partial_{\beta \alpha} \mathbf{a}^{\tau}=\partial_{\alpha}\left(-\Gamma_{\beta \sigma}^{\tau} \mathbf{a}^{\sigma}+b_{\beta}^{\tau} \mathbf{a}^{3}\right)-\partial_{\beta}\left(-\Gamma_{\alpha \sigma}^{\tau} \mathbf{a}^{\sigma}+b_{\alpha}^{\tau} \mathbf{a}^{3}\right) \\
= & -\left(\partial_{\alpha} \Gamma_{\beta \sigma}^{\tau}\right) \mathbf{a}^{\sigma}+\Gamma_{\beta \sigma}^{\tau} \Gamma_{\alpha \nu}^{\sigma} \mathbf{a}^{\nu}-\Gamma_{\beta \sigma}^{\tau} \sigma_{\alpha}^{\sigma} \mathbf{a}^{3}+\left(\partial_{\alpha} b_{\beta}^{\tau}\right) \mathbf{a}^{3}-b_{\beta}^{\tau} b_{\alpha \sigma} \mathbf{a}^{\sigma} \\
& +\left(\partial_{\beta} \Gamma_{\alpha \sigma}^{\tau}\right) \mathbf{a}^{\sigma}-\Gamma_{\alpha \sigma}^{\tau} \Gamma_{\beta \mu}^{\sigma} \mathbf{a}^{\mu}+\Gamma_{\alpha \sigma}^{\tau} b_{\beta}^{\sigma} \mathbf{a}^{3}-\left(\partial_{\beta} b_{\alpha}^{\tau}\right) \mathbf{a}^{3}+b_{\alpha}^{\tau} b_{\beta \sigma} \mathbf{a}^{\sigma} .
\end{aligned}
$$

Consequently,

$$
0=\left(\partial_{\alpha \beta} \mathbf{a}^{\tau}-\partial_{\beta \alpha} \mathbf{a}^{\tau}\right) \cdot \mathbf{a}^{3}=\partial_{\alpha} b_{\beta}^{\tau}-\partial_{\beta} b_{\alpha}^{\tau}+\Gamma_{\alpha \sigma}^{\tau} b_{\beta}^{\sigma}-\Gamma_{\beta \sigma}^{\tau} b_{\alpha}^{\sigma},
$$

on the one hand. On the other hand, we immediately infer from the definition of the functions $\left.b_{\beta}^{\tau}\right|_{\alpha}$ that we also have

$$
\left.b_{\beta}^{\tau}\right|_{\alpha}-\left.b_{\alpha}^{\tau}\right|_{\beta}=\partial_{\alpha} b_{\beta}^{\tau}-\partial_{\beta} b_{\alpha}^{\tau}+\Gamma_{\alpha \sigma}^{\tau} b_{\beta}^{\sigma}-\Gamma_{\beta \sigma}^{\tau} b_{\alpha}^{\sigma},
$$

and thus the proof is complete. 
The functions $\rho_{\alpha \beta}(\boldsymbol{\eta})$ are called the covariant components of the linearized change of curvature tensor associated with a displacement $\boldsymbol{\eta}=\eta_{i} \mathbf{a}^{i}$ of the surface $S$. The functions

$$
\eta_{3 \mid \alpha \beta}=\partial_{\alpha \beta} \eta_{3}-\Gamma_{\alpha \beta}^{\sigma} \partial_{\sigma} \eta_{3} \text { and }\left.b_{\beta}^{\tau}\right|_{\alpha}=\partial_{\alpha} b_{\beta}^{\tau}+\Gamma_{\alpha \sigma}^{\tau} b_{\beta}^{\sigma}-\Gamma_{\alpha \beta}^{\sigma} b_{\sigma}^{\tau}
$$

respectively represent a second-order covariant derivative of the vector field $\eta_{i} \mathbf{a}^{i}$ and a first-order covariant derivative of the second fundamental form of $S$, defined here by means of its mixed components $b_{\beta}^{\tau}$.

Remarks. (1) The functions $b_{\alpha \beta}(\boldsymbol{\eta})$ are not always well defined (in order that they be, the vectors $a_{\alpha}(\boldsymbol{\eta})$ must be linearly independent in $\left.\bar{\omega}\right)$, but the functions $\rho_{\alpha \beta}(\boldsymbol{\eta})$ are always well defined.

(2) The symmetry $\rho_{\alpha \beta}(\boldsymbol{\eta})=\rho_{\beta \alpha}(\boldsymbol{\eta})$ follows immediately by inspection of the expression $\rho_{\alpha \beta}(\boldsymbol{\eta})=\left(\partial_{\alpha \beta} \boldsymbol{\eta}-\Gamma_{\alpha \beta}^{\sigma} \partial_{\sigma} \boldsymbol{\eta}\right) \cdot \mathbf{a}_{3}$ found there. By contrast, deriving the same symmetry from the other expression of $\rho_{\alpha \beta}(\boldsymbol{\eta})$ requires proving first that the covariant derivatives $\left.b_{\beta}^{\sigma}\right|_{\alpha}$ are themselves symmetric with respect to the indices $\alpha$ and $\beta$ (cf. part (v) of the proof of Theorem 2.8-1).

While the expression of the components $\rho_{\alpha \beta}(\boldsymbol{\eta})$ in terms of the covariant components $\eta_{i}$ of the displacement field is fairly complicated but well known (see, e.g., Koiter [60]), that in terms of $\boldsymbol{\eta}=\eta_{i} \mathbf{a}^{i}$ is remarkably simple but seems to have been mostly ignored, although it already appeared in Bamberger [10]. Together with the expression of the components $\gamma_{\alpha \beta}(\boldsymbol{\eta})$ in terms of $\boldsymbol{\eta}$ (Theorem 2.8-1), this simpler expression was efficiently put to use by Blouza \& Le Dret [13], who showed that their principal merit is to afford the definition of the components $\gamma_{\alpha \beta}(\boldsymbol{\eta})$ and $\rho_{\alpha \beta}(\boldsymbol{\eta})$ under substantially weaker regularity assumptions on the mapping $\boldsymbol{\theta}$.

More specifically, we were led to assume that $\boldsymbol{\theta} \in \mathcal{C}^{3}\left(\bar{\omega} ; \mathbb{R}^{3}\right)$ in Theorem 2.8-2 in order to insure that $\rho_{\alpha \beta}(\boldsymbol{\eta}) \in L^{2}(\omega)$ if $\boldsymbol{\eta}=\eta_{i} \mathbf{a}^{i}$ with $\eta_{\alpha} \in H^{1}(\omega)$ and $\eta_{3} \in H^{2}(\omega)$. The culprits responsible for this regularity are the functions $\left.b_{\beta}^{\tau}\right|_{\alpha}$ appearing in the functions $\rho_{\alpha \beta}(\boldsymbol{\eta})$. Otherwise Blouza \& Le Dret [13] have shown how this regularity assumption on $\boldsymbol{\theta}$ can be weakened if only the expressions of $\gamma_{\alpha \beta}(\boldsymbol{\eta})$ and $\rho_{\alpha \beta}(\boldsymbol{\eta})$ in terms of the field $\boldsymbol{\eta}$ are considered.

We are now in a position to describe the linear Koiter shell equations. Let $\gamma_{0}$ be a measurable subset of $\gamma=\partial \omega$ that satisfies length $\gamma_{0}>0$, let $\partial_{\nu}$ denote the outer normal derivative operator along $\partial \omega$, and let the space $\boldsymbol{V}(\omega)$ be defined by

$$
\boldsymbol{V}(\omega):=\left\{\boldsymbol{\eta}=\eta_{i} \mathbf{a}^{i} ; \eta_{\alpha} \in H^{1}(\omega), \eta_{3} \in H^{2}(\omega), \eta_{i}=\partial_{\nu} \eta_{3}=0 \text { on } \gamma_{0}\right\} .
$$

Then the displacement field $\boldsymbol{\zeta}^{\varepsilon}=\zeta_{i}^{\varepsilon} \mathbf{a}^{i}$ of the middle surface $S=\boldsymbol{\theta}(\bar{\omega})$ of the shell (the covariant components $\zeta_{i}^{\varepsilon}$ are unknown) should be a stationary point over the space $\boldsymbol{V}(\omega)$ of the functional $j$ defined by

$$
\begin{aligned}
j(\boldsymbol{\eta})= & \frac{1}{2} \int_{\omega}\left\{\varepsilon a^{\alpha \beta \sigma \tau} \gamma_{\sigma \tau}(\boldsymbol{\eta}) \gamma_{\alpha \beta}(\boldsymbol{\eta})+\frac{\varepsilon^{3}}{3} a^{\alpha \beta \sigma \tau} \rho_{\sigma \tau}(\boldsymbol{\eta}) \rho_{\alpha \beta}(\boldsymbol{\eta})\right\} \sqrt{a} d y \\
& -\int_{\omega} p^{i, \varepsilon} \eta_{i} \sqrt{a} d y
\end{aligned}
$$

for all $\boldsymbol{\eta}=\eta_{i} \mathbf{a}^{i} \in \boldsymbol{V}(\omega)$. This functional $j$ is called Koiter's energy for a linearly elastic shell. 
Equivalently, the vector field $\boldsymbol{\zeta}^{\varepsilon}=\zeta_{i}^{\varepsilon} \mathbf{a}^{i} \in \boldsymbol{V}(\omega)$ should satisfy the variational equations

$$
\begin{aligned}
\int_{\omega}\left\{\varepsilon a^{\alpha \beta \sigma \tau} \gamma_{\sigma \tau}\left(\boldsymbol{\zeta}^{\varepsilon}\right) \gamma_{\alpha \beta}(\boldsymbol{\eta})\right. & \left.+\frac{\varepsilon^{3}}{3} a^{\alpha \beta \sigma \tau} \rho_{\sigma \tau}\left(\boldsymbol{\zeta}^{\varepsilon}\right) \rho_{\alpha \beta}(\boldsymbol{\eta})\right\} \sqrt{a} d y \\
= & \int_{\omega} p^{i, \varepsilon} \eta_{i} \sqrt{a} d y \text { for all } \boldsymbol{\eta}=\eta_{i} \mathbf{a}^{i} \in \boldsymbol{V}(\omega) .
\end{aligned}
$$

We recall that the functions

$$
a^{\alpha \beta \sigma \tau}:=\frac{4 \lambda \mu}{\lambda+2 \mu} a^{\alpha \beta} a^{\sigma \tau}+2 \mu\left(a^{\alpha \sigma} a^{\beta \tau}+a^{\alpha \tau} a^{\beta \sigma}\right)
$$

denote the contravariant components of the shell elasticity tensor ( $\lambda$ and $\mu$ are the Lamé constants of the elastic material constituting the shell), $\gamma_{\alpha \beta}(\boldsymbol{\eta})$ and $\rho_{\alpha \beta}(\boldsymbol{\eta})$ denote the covariant components of the linearized change of metric, and change of curvature, tensors associated with a displacement field $\boldsymbol{\eta}=\eta_{i} \mathbf{a}^{i}$ of $S$, and the given functions $p^{i, \varepsilon} \in L^{2}(\omega)$ account for the applied forces. Finally, the boundary conditions $\eta_{i}=\partial_{\nu} \eta_{3}=0$ on $\gamma_{0}$ express that the shell is clamped along the portion $\boldsymbol{\theta}\left(\gamma_{0}\right)$ of its middle surface (see Figure 2.4-1).

The choice of the function spaces $H^{1}(\omega)$ and $H^{2}(\omega)$ for the tangential components $\eta_{\alpha}$ and normal components $\eta_{3}$ of the displacement fields $\boldsymbol{\eta}=\eta_{i} \mathbf{a}^{i}$ is guided by the natural requirement that the functions $\gamma_{\alpha \beta}(\boldsymbol{\eta})$ and $\rho_{\alpha \beta}(\boldsymbol{\eta})$ be both in $L^{2}(\omega)$, so that the energy is in turn well defined for $\boldsymbol{\eta} \in \boldsymbol{V}(\omega)$. Otherwise these choices can be weakened to accommodate shells whose middle surfaces have little regularity (see Blouza \& Le Dret [13]).

Remark. Koiter's linear equations can be fully justified by means of an asymptotic analysis of the "three-dimensional" equations of linearized elasticity as $\varepsilon \rightarrow 0$; see Section 2.6. For more details, see Ciarlet [20], Chapter 7 and the references therein.

\subsection{KORN'S INEQUALITIES ON A SURFACE}

Our objective in the next sections is to study the existence and uniqueness of the solution to the variational equations associated with the linear Koiter model. To this end, we shall see (Theorem 2.10-1) that, under the assumptions $3 \lambda+2 \mu>0$ and $\mu>0$, there exists a constant $c_{e}>0$ such that

$$
\sum_{\alpha, \beta}\left|t_{\alpha \beta}\right|^{2} \leq c_{e} a^{\alpha \beta \sigma \tau}(y) t_{\sigma \tau} t_{\alpha \beta}
$$

for all $y \in \bar{\omega}$ and all symmetric matrices $\left(t_{\alpha \beta}\right)$. When length $\gamma_{0}>0$, the existence and uniqueness of a solution to this variational problem by means of the LaxMilgram lemma will then be a consequence of the existence of a constant $c$ such that

$$
\begin{aligned}
& \left\{\sum_{\alpha}\left\|\eta_{\alpha}\right\|_{H^{1}(\omega)}^{2}+\left\|\eta_{3}\right\|_{H^{2}(\omega)}^{2}\right\}^{1 / 2} \\
& \leq c\left\{\sum_{\alpha, \beta}\left\|\gamma_{\alpha \beta}(\boldsymbol{\eta})\right\|_{L^{2}(\omega)}^{2}+\sum_{\alpha, \beta}\left\|\rho_{\alpha \beta}(\boldsymbol{\eta})\right\|_{L^{2}(\omega)}^{2}\right\}^{1 / 2} \text { for all } \boldsymbol{\eta}=\eta_{i} \mathbf{a}^{i} \in \boldsymbol{V}(\omega) .
\end{aligned}
$$


Such a key inequality is an instance of a Korn inequality on a surface. The objective of this section is to establish such an inequality.

To begin with, we establish a Korn's inequality on a surface, "without boundary conditions", as a consequence of the lemma of J.L. Lions (cf. Theorem 1.5-1). We follow here Ciarlet \& Miara [33] (see also Bernadou, Ciarlet \& Miara $[12])$.

Theorem 2.9-1. Let $\omega$ be a domain in $\mathbb{R}^{2}$ and let $\boldsymbol{\theta} \in \mathcal{C}^{3}\left(\bar{\omega} ; \mathbb{R}^{3}\right)$ be an injective immersion. Given $\boldsymbol{\eta}=\eta_{i} \mathbf{a}^{i}$ with $\eta_{\alpha} \in H^{1}(\omega)$ and $\eta_{3} \in H^{2}(\omega)$, let

$$
\begin{aligned}
& \gamma_{\alpha \beta}(\boldsymbol{\eta}):=\left\{\frac{1}{2}\left(\partial_{\beta} \boldsymbol{\eta} \cdot \mathbf{a}_{\alpha}+\partial_{\alpha} \boldsymbol{\eta} \cdot \mathbf{a}_{\beta}\right)\right\} \in L^{2}(\omega), \\
& \rho_{\alpha \beta}(\boldsymbol{\eta}):=\left\{\left(\partial_{\alpha \beta} \boldsymbol{\eta}-\Gamma_{\alpha \beta}^{\sigma} \partial_{\sigma} \boldsymbol{\eta}\right) \cdot \mathbf{a}_{3}\right\} \in L^{2}(\omega)
\end{aligned}
$$

denote the covariant components of the linearized change of metric, and linearized change of curvature, tensors associated with the displacement field $\boldsymbol{\eta}=\eta_{i} \mathbf{a}^{i}$ of the surface $S=\boldsymbol{\theta}(\bar{\omega})$. Then there exists a constant $c_{0}=c_{0}(\omega, \boldsymbol{\theta})$ such that

$$
\begin{aligned}
& \left\{\sum_{\alpha}\left\|\eta_{\alpha}\right\|_{H^{1}(\omega)}^{2}+\left\|\eta_{3}\right\|_{H^{2}(\omega)}^{2}\right\}^{1 / 2} \\
& \quad \leq c_{0}\left\{\sum_{\alpha}\left\|\eta_{\alpha}\right\|_{L^{2}(\omega)}^{2}+\left\|\eta_{3}\right\|_{H^{1}(\omega)}^{2}+\sum_{\alpha, \beta}\left\|\gamma_{\alpha \beta}(\boldsymbol{\eta})\right\|_{L^{2}(\omega)}^{2}+\sum_{\alpha, \beta}\left\|\rho_{\alpha \beta}(\boldsymbol{\eta})\right\|_{L^{2}(\omega)}^{2}\right\}^{1 / 2}
\end{aligned}
$$

for all $\boldsymbol{\eta}=\eta_{i} \mathbf{a}^{i}$ with $\eta_{\alpha} \in H^{1}(\omega)$ and $\eta_{3} \in H^{2}(\omega)$

Proof. The "fully explicit" expressions of the functions $\gamma_{\alpha \beta}(\boldsymbol{\eta})$ and $\rho_{\alpha \beta}(\boldsymbol{\eta})$, as found in Theorems 2.8-1 and 2.8-2, are used in this proof, simply because they are more convenient for its purposes.

(i) Define the space

$$
\begin{aligned}
\boldsymbol{W}(\omega):=\left\{\boldsymbol{\eta}=\eta_{i} \mathbf{a}^{i} ; \eta_{\alpha} \in L^{2}(\omega), \eta_{3} \in H^{1}(\omega),\right. \\
\\
\left.\gamma_{\alpha \beta}(\boldsymbol{\eta}) \in L^{2}(\omega), \rho_{\alpha \beta}(\boldsymbol{\eta}) \in L^{2}(\omega)\right\} .
\end{aligned}
$$

Then, equipped with the norm $\|\cdot\|_{\boldsymbol{W}(\omega)}$ defined by

$\|\boldsymbol{\eta}\|_{\boldsymbol{W}(\omega)}:=\left\{\sum_{\alpha}\left\|\eta_{\alpha}\right\|_{L^{2}(\omega)}^{2}+\left\|\eta_{3}\right\|_{H^{1}(\omega)}^{2}+\sum_{\alpha, \beta}\left\|\gamma_{\alpha \beta}(\boldsymbol{\eta})\right\|_{L^{2}(\omega)}^{2}+\sum_{\alpha, \beta}\left\|\rho_{\alpha \beta}(\boldsymbol{\eta})\right\|_{L^{2}(\omega)}^{2}\right\}^{1 / 2}$

the space $\boldsymbol{W}(\omega)$ is a Hilbert space.

The relations " $\gamma_{\alpha \beta}(\boldsymbol{\eta}) \in L^{2}(\omega)$ " and " $\rho_{\alpha \beta}(\boldsymbol{\eta}) \in L^{2}(\omega)$ " appearing in the definition of the space $\boldsymbol{W}(\omega)$ are to be understood in the sense of distributions. They mean that a vector field $\boldsymbol{\eta}=\eta_{i} \mathbf{a}^{i}$, with $\eta_{\alpha} \in L^{2}(\omega)$ and $\eta_{3} \in H^{1}(\omega)$, belongs to $\boldsymbol{W}(\omega)$ if there exist functions in $L^{2}(\omega)$, denoted $\gamma_{\alpha \beta}(\boldsymbol{\eta})$ and $\rho_{\alpha \beta}(\boldsymbol{\eta})$, such that for 
all $\varphi \in \mathcal{D}(\omega)$

$$
\begin{aligned}
\int_{\omega} \gamma_{\alpha \beta}(\boldsymbol{\eta}) \varphi d y=-\int_{\omega}\{ & \left\{\frac{1}{2}\left(\eta_{\beta} \partial_{\alpha} \varphi+\eta_{\alpha} \partial_{\beta} \varphi\right)+\Gamma_{\alpha \beta}^{\sigma} \eta_{\sigma} \varphi+b_{\alpha \beta} \eta_{3} \varphi\right\} d y \\
\int_{\omega} \rho_{\alpha \beta}(\boldsymbol{\eta}) \varphi d y=-\int_{\omega}\{ & \partial_{\alpha} \eta_{3} \partial_{\beta} \varphi+\Gamma_{\alpha \beta}^{\sigma} \partial_{\sigma} \eta_{3} \varphi+b_{\alpha}^{\sigma} b_{\sigma \beta} \eta_{3} \varphi \\
& +\eta_{\sigma} \partial_{\beta}\left(b_{\alpha}^{\sigma} \varphi\right)+b_{\alpha}^{\sigma} \Gamma_{\beta \sigma}^{\tau} \eta_{\tau} \varphi \\
& +\eta_{\tau} \partial_{\alpha}\left(b_{\beta}^{\tau} \varphi\right)+b_{\beta}^{\tau} \Gamma_{\alpha \tau}^{\sigma} \eta_{\sigma} \varphi \\
& \left.-\left(\partial_{\alpha} b_{\beta}^{\tau}+\Gamma_{\alpha \sigma}^{\tau} b_{\beta}^{\sigma}-\Gamma_{\alpha \beta}^{\sigma} b_{\sigma}^{\tau}\right) \eta_{\tau} \varphi\right\} d y
\end{aligned}
$$

Let there be given a Cauchy sequence $\left(\boldsymbol{\eta}^{k}\right)_{k=1}^{\infty}$ with elements $\boldsymbol{\eta}^{k}=\eta_{i}^{k} \mathbf{a}^{i} \in$ $\boldsymbol{W}(\omega)$. The definition of the norm $\|\cdot\|_{\boldsymbol{W}(\omega)}$ shows that there exist $\eta_{\alpha} \in L^{2}(\omega)$, $\eta_{3} \in H^{1}(\omega), \gamma_{\alpha \beta} \in L^{2}(\omega)$, and $\rho_{\alpha \beta} \in L^{2}(\omega)$ such that

$$
\begin{aligned}
\eta_{\alpha}^{k} \rightarrow \eta_{\alpha} \text { in } L^{2}(\omega), & \eta_{3}^{k} \rightarrow \eta_{3} \text { in } H^{1}(\omega), \\
\gamma_{\alpha \beta}\left(\boldsymbol{\eta}^{k}\right) \rightarrow \gamma_{\alpha \beta} \text { in } L^{2}(\omega), & \rho_{\alpha \beta}\left(\boldsymbol{\eta}^{k}\right) \rightarrow \rho_{\alpha \beta} \text { in } L^{2}(\omega)
\end{aligned}
$$

as $k \rightarrow \infty$. Given a function $\varphi \in \mathcal{D}(\omega)$, letting $k \rightarrow \infty$ in the relations

$$
\int_{\omega} \gamma_{\alpha \beta}\left(\boldsymbol{\eta}^{k}\right) \varphi d \omega=\ldots \text { and } \int_{\omega} \rho_{\alpha \beta}\left(\boldsymbol{\eta}^{k}\right) \varphi d \omega=\ldots
$$

then shows that $\gamma_{\alpha \beta}=\gamma_{\alpha \beta}(\boldsymbol{\eta})$ and $\rho_{\alpha \beta}=\rho_{\alpha \beta}(\boldsymbol{\eta})$.

(ii) The spaces $\boldsymbol{W}(\omega)$ and $\left\{\boldsymbol{\eta}=\eta_{i} \mathbf{a}^{i} ; \eta_{\alpha} \in H^{1}(\omega), \eta_{3} \in H^{2}(\omega)\right\}$ coincide.

Clearly, $\left\{\boldsymbol{\eta}=\eta_{i} \mathbf{a}^{i} ; \eta_{\alpha} \in H^{1}(\omega), \eta_{3} \in H^{2}(\omega)\right\} \subset \boldsymbol{W}(\omega)$. To prove the other inclusion, let $\boldsymbol{\eta}=\eta_{i} \mathbf{a}^{i} \in \boldsymbol{W}(\omega)$. The relations

$$
s_{\alpha \beta}(\boldsymbol{\eta}):=\frac{1}{2}\left(\partial_{\alpha} \eta_{\beta}+\partial_{\beta} \eta_{\alpha}\right)=\gamma_{\alpha \beta}(\boldsymbol{\eta})+\Gamma_{\alpha \beta}^{\sigma} \eta_{\sigma}+b_{\alpha \beta} \eta_{3}
$$

then imply that $s_{\alpha \beta}(\boldsymbol{\eta}) \in L^{2}(\omega)$ since the functions $\Gamma_{\alpha \beta}^{\sigma}$ and $b_{\alpha \beta}$ are continuous on $\bar{\omega}$. Therefore,

$$
\begin{aligned}
\partial_{\sigma} \eta_{\alpha} & \in H^{-1}(\omega), \\
\partial_{\beta}\left(\partial_{\sigma} \eta_{\alpha}\right)=\left\{\partial_{\beta} s_{\alpha \sigma}(\boldsymbol{\eta})+\partial_{\sigma} s_{\alpha \beta}(\boldsymbol{\eta})-\partial_{\alpha} s_{\beta \sigma}(\boldsymbol{\eta})\right\} & \in H^{-1}(\omega),
\end{aligned}
$$

since $\chi \in L^{2}(\omega)$ implies $\partial_{\sigma} \chi \in H^{-1}(\omega)$. Hence $\partial_{\sigma} \eta_{\alpha} \in L^{2}(\omega)$ by the lemma of J.L. Lions (Theorem 1.5-1) and thus $\eta_{\alpha} \in H^{1}(\omega)$.

The definition of the functions $\rho_{\alpha \beta}(\boldsymbol{\eta})$, the continuity over $\bar{\omega}$ of the functions $\Gamma_{\alpha \beta}^{\sigma}, b_{\sigma \beta}, b_{\alpha}^{\sigma}$, and $\partial_{\alpha} b_{\beta}^{\tau}$, and the relations $\rho_{\alpha \beta}(\boldsymbol{\eta}) \in L^{2}(\omega)$ then imply that $\partial_{\alpha \beta} \eta_{3} \in$ $L^{2}(\omega)$, hence that $\eta_{3} \in H^{2}(\omega)$.

(iii) Korn's inequality without boundary conditions.

The identity mapping $\iota$ from the space $\left\{\boldsymbol{\eta}=\eta_{i} \mathbf{a}^{i} ; \eta_{\alpha} \in H^{1}(\omega), \eta_{3} \in H^{2}(\omega)\right\}$ equipped with the norm $\boldsymbol{\eta}=\eta_{i} \mathbf{a}^{i} \mapsto\left\{\sum_{\alpha}\left\|\eta_{\alpha}\right\|_{H^{1}(\omega)}^{2}+\left\|\eta_{3}\right\|_{H^{2}(\omega)}^{2}\right\}^{1 / 2}$ into the space $\boldsymbol{W}(\omega)$ equipped with $\|\cdot\|_{\boldsymbol{W}(\omega)}$ is injective, continuous, and surjective by (ii). Since both spaces are complete (cf. (i)), the open mapping theorem then shows that the inverse mapping $\iota^{-1}$ is also continuous or equivalently, that the inequality of Korn's type without boundary conditions holds.

In order to establish a Korn's inequality "with boundary conditions", we have to identify classes of boundary conditions to be imposed on the fields $\boldsymbol{\eta}=\eta_{i} \mathbf{a}^{i}$, with 
$\eta_{\alpha} \in H^{1}(\omega)$ and $\eta_{3} \in H^{2}(\omega)$, in order that we can "get rid" of the norms $\left\|\eta_{\alpha}\right\|_{L^{2}(\omega)}$ and $\left\|\eta_{3}\right\|_{H^{1}(\omega)}$ in the right-hand side of the above inequality, i.e., situations where the semi-norm

$$
\boldsymbol{\eta}=\eta_{i} \mathbf{a}^{i} \rightarrow\left\{\sum_{\alpha, \beta}\left\|\gamma_{\alpha \beta}(\boldsymbol{\eta})\right\|_{L^{2}(\omega)}^{2}+\sum_{\alpha, \beta}\left\|\rho_{\alpha \beta}(\boldsymbol{\eta})\right\|_{L^{2}(\omega)}^{2}\right\}^{1 / 2}
$$

becomes a norm, which should be in addition equivalent to the norm

$$
\boldsymbol{\eta}=\eta_{i} \mathbf{a}^{i} \mapsto\left\{\sum_{\alpha}\left\|\eta_{\alpha}\right\|_{H^{1}(\omega)}^{2}+\left\|\eta_{3}\right\|_{H^{2}(\omega)}^{2}\right\}^{1 / 2} .
$$

To this end, we need an infinitesimal rigid displacement lemma "on a surface" (the adjective "infinitesimal" reminds that only the linearized parts $\gamma_{\alpha \beta}(\boldsymbol{\eta})$ and $\rho_{\alpha \beta}(\boldsymbol{\eta})$ of the "full" change of metric and curvature tensors $\frac{1}{2}\left(a_{\alpha \beta}(\boldsymbol{\eta})-a_{\alpha \beta}\right)$ and $\left(b_{\alpha \beta}(\boldsymbol{\eta})-b_{\alpha \beta}\right)$ are required to vanish in $\left.\omega\right)$, which is due to Bernadou \& Ciarlet [11], Theorems 5.1-1 and 5.2-1; see also Bernadou, Ciarlet \& Miara [12], Lemmas 2.5 and 2.6 and Blouza \& Le Dret [13], Theorem 6.

Part (a) in the next theorem is an infinitesimal rigid displacement lemma on a surface, "without boundary conditions", while part (b) is an infinitesimal rigid displacement lemma on a surface, "with boundary conditions".

Any proof of this theorem is, at least to some extent, delicate. The one given here is not the shortest, but it is a natural one: It relies on the classical, and much easier to prove, "three-dimensional infinitesimal rigid displacement lemma in Cartesian coordinates" used in part (iii) of the next proof (a "direct" proof, such as the one originally found by Bernadou \& Ciarlet [11], is surprisingly "technical").

In the next proof, the functions $\widehat{e}_{i j}(\widehat{\boldsymbol{v}})$ and $e_{i j}(\boldsymbol{v})$ are the Cartesian and covariant components of the "three-dimensional" linearized strain tensor respectively associated with a displacement field $\widehat{v}_{i} \widehat{\mathbf{e}}^{i}:\{\widehat{\Omega}\}^{-} \rightarrow \mathbb{R}^{3}$ and a displacement field $v_{i} \boldsymbol{g}^{i}: \bar{\Omega} \rightarrow \mathbb{R}^{3}$; the functions $\Gamma_{i j}^{p}$ are the Christoffel symbols (of the second kind) associated with the mapping $\Theta: \bar{\Omega} \rightarrow\{\widehat{\Omega}\}^{-}$; finally, the functions $v_{i \| j}$ are the covariant derivatives of the vector field $v_{i} \boldsymbol{g}^{i}: \bar{\Omega} \rightarrow \mathbb{R}^{3}$.

Theorem 2.9-2. Let there be given a domain $\omega$ in $\mathbb{R}^{2}$ and an injective immersion $\boldsymbol{\theta} \in \mathcal{C}^{3}\left(\bar{\omega} ; \mathbb{R}^{3}\right)$.

(a) Let $\boldsymbol{\eta}=\eta_{i} \mathbf{a}^{i}$ with $\eta_{\alpha} \in H^{1}(\omega)$ and $\eta_{3} \in H^{2}(\omega)$ be such that

$$
\gamma_{\alpha \beta}(\boldsymbol{\eta})=\rho_{\alpha \beta}(\boldsymbol{\eta})=0 \text { in } \omega .
$$

Then there exist two vectors $\mathbf{a}, \boldsymbol{b} \in \mathbb{R}^{3}$ such that

$$
\boldsymbol{\eta}(y)=\mathbf{a}+\boldsymbol{b} \wedge \boldsymbol{\theta}(y) \text { for all } y \in \bar{\omega} .
$$

(b) Let $\gamma_{0}$ be a $\mathrm{d} \gamma$-measurable subset of $\gamma=\partial \omega$ that satisfies length $\gamma_{0}>0$ and let a vector field $\boldsymbol{\eta}=\eta_{i} \mathbf{a}^{i}$ with $\eta_{\alpha} \in H^{1}(\omega)$ and $\eta_{3} \in H^{2}(\omega)$ be such that

$$
\gamma_{\alpha \beta}(\boldsymbol{\eta})=\rho_{\alpha \beta}(\boldsymbol{\eta})=0 \text { in } \omega \text { and } \eta_{i}=\partial_{\nu} \eta_{3}=0 \text { on } \gamma_{0} .
$$

Then $\boldsymbol{\eta}=\mathbf{0}$ in $\omega$.

Proof. The proof is divided into five parts, numbered (i) to (v).

(i) In parts (i) to (iii), $\Omega$ denotes a domain in $\mathbb{R}^{3}$ and $\Theta: \bar{\Omega} \rightarrow \mathbb{R}^{3}$ denotes a $\mathcal{C}^{2}$-diffeomorphism from $\bar{\Omega}$ onto its image $\Theta(\bar{\Omega})$. Consequently, the three vectors $\boldsymbol{g}_{i}(x):=\partial_{i} \boldsymbol{\Theta}(x)$, where $\partial_{i}=\partial / \partial x_{i}$, are linearly independent at all points $x=$ 
$\left(x_{i}\right) \in \bar{\Omega}$ and the three vectors $\boldsymbol{g}^{i}(x)$ defined by $\boldsymbol{g}^{i}(x) \boldsymbol{g}_{j}(x)=\delta_{j}^{i}$ are likewise linearly independent at all points $x \in \bar{\Omega}$. Note also that $\boldsymbol{g}_{i} \in \mathcal{C}^{1}\left(\bar{\Omega} ; \mathbb{R}^{3}\right)$ and $\boldsymbol{g}^{i} \in \mathcal{C}^{1}\left(\bar{\Omega} ; \mathbb{R}^{3}\right)$.

Let $\widehat{\mathbf{e}}_{i}$ denote the basis of $\mathbb{R}^{3}$, let $\widehat{x}_{i}$ denote the Cartesian coordinates of a point $\widehat{x} \in \mathbb{R}^{3}$, let $\widehat{\partial}_{i}:=\partial / \partial \widehat{x}_{i}$, and let $\widehat{\Omega}:=\boldsymbol{\Theta}(\Omega)$. With any vector field $\boldsymbol{v}=v_{i} \boldsymbol{g}^{i}: \bar{\Omega} \rightarrow$ $\mathbb{R}^{3}$ with $v_{i} \in H^{1}(\Omega)$, we then associate a vector field $\widehat{\boldsymbol{v}}=\widehat{v}_{i} \widehat{\mathbf{e}}^{i}:\{\widehat{\Omega}\}^{-} \rightarrow \mathbb{R}^{3}$ by letting

$$
\widehat{v}_{i}(\widehat{x}) \widehat{\mathbf{e}}^{i}=v_{i}(x) \boldsymbol{g}^{i}(x) \text { for all } \widehat{x}=\boldsymbol{\Theta}(x), x \in \bar{\Omega} .
$$

It is then clear that $\widehat{v}_{i} \in H^{1}(\widehat{\Omega})$.

We now show that, for all $x \in \Omega$,

$$
\widehat{\partial}_{j} \widehat{v}_{i}(\widehat{x})=\left(v_{k \| \ell}\left[\boldsymbol{g}^{k}\right]_{i}\left[\boldsymbol{g}^{\ell}\right]_{j}\right)(x), \widehat{x}=\boldsymbol{\Theta}(x),
$$

where

$$
v_{i \| j}:=\partial_{j} v_{i}-\Gamma_{i j}^{p} v_{p} \text { and } \Gamma_{i j}^{p}:=\boldsymbol{g}^{p} \cdot \partial_{i} \boldsymbol{g}_{j},
$$

and

$$
\left[\boldsymbol{g}^{k}(x)\right]_{i}:=\boldsymbol{g}^{k}(x) \cdot \widehat{\mathbf{e}}_{i}
$$

denotes the $i$-th component of $\boldsymbol{g}^{k}(x)$ over the basis $\left\{\widehat{\mathbf{e}}_{1}, \widehat{\mathbf{e}}_{2}, \widehat{\mathbf{e}}_{3}\right\}$.

In what follows, the simultaneous appearance of $\widehat{x}$ and $x$ in an equality means that they are related by $\widehat{x}=\boldsymbol{\Theta}(x)$ and that the equality in question holds for all $x \in \Omega$.

Let $\boldsymbol{\Theta}(x)=\Theta^{k}(x) \widehat{\mathbf{e}}_{k}$ and $\widehat{\boldsymbol{\Theta}}(\widehat{x})=\widehat{\Theta}^{i}(\widehat{x}) \mathbf{e}_{i}$, where $\widehat{\boldsymbol{\Theta}}: \widehat{\Omega} \rightarrow \mathbb{R}^{3}$ denotes the inverse mapping of $\Theta: \Omega \rightarrow \mathbb{R}^{3}$. Since $\widehat{\boldsymbol{\Theta}}(\boldsymbol{\Theta}(x))=x$ for all $x \in \Omega$, the chain rule shows that the matrices $\boldsymbol{\nabla} \boldsymbol{\Theta}(x):=\left(\partial_{j} \Theta^{k}(x)\right)$ (the row index is $k$ ) and $\widehat{\nabla} \widehat{\boldsymbol{\Theta}}(\widehat{x}):=\left(\widehat{\partial}_{k} \widehat{\Theta}^{i}(\widehat{x})\right)$ (the row index is $i$ ) satisfy

$$
\widehat{\nabla} \widehat{\Theta}(\widehat{x}) \nabla \Theta(x)=\boldsymbol{I},
$$

or equivalently,

$$
\widehat{\partial}_{k} \widehat{\Theta}^{i}(\widehat{x}) \partial_{j} \Theta^{k}(x)=\left(\begin{array}{lll}
\widehat{\partial}_{1} \widehat{\Theta}^{i}(\widehat{x}) & \partial_{2} \widehat{\Theta}^{i}(\widehat{x}) & \partial_{3} \widehat{\Theta}^{i}(\widehat{x})
\end{array}\right)\left(\begin{array}{c}
\partial_{j} \Theta^{1}(x) \\
\partial_{j} \Theta^{2}(x) \\
\partial_{j} \Theta^{3}(x)
\end{array}\right)=\delta_{j}^{i} .
$$

The components of the above column vector being precisely those of the vector $\boldsymbol{g}_{j}(x)$, the components of the above row vector must be those of the vector $\boldsymbol{g}^{i}(x)$ since $\boldsymbol{g}^{i}(x)$ is uniquely defined for each exponent $i$ by the three relations $\boldsymbol{g}^{i}(x)$. $\boldsymbol{g}_{j}(x)=\delta_{j}^{i}, j=1,2,3$. Hence the $k$-th component of $\boldsymbol{g}^{i}(x)$ over the basis $\left\{\widehat{\mathbf{e}}_{1}, \widehat{\mathbf{e}}_{2}, \widehat{\mathbf{e}}_{3}\right\}$ can be also expressed in terms of the inverse mapping $\widehat{\boldsymbol{\Theta}}$, as:

$$
\left[\boldsymbol{g}^{i}(x)\right]_{k}=\widehat{\partial}_{k} \widehat{\Theta}^{i}(\widehat{x}) .
$$

We next compute the derivatives $\partial_{\ell} \boldsymbol{g}^{q}(x)$ (it is easily seen that the fields $\boldsymbol{g}^{q}=g^{q r} \boldsymbol{g}_{r}$ are of class $\mathcal{C}^{1}$ on $\Omega$ since $\Theta$ is assumed to be of class $\mathcal{C}^{2}$ ). These derivatives will be needed below for expressing the derivatives $\widehat{\partial}_{j} \widehat{u}_{i}(\widehat{x})$ as functions of $x$ (recall that $\left.\widehat{u}_{i}(\widehat{x})=u_{k}(x)\left[\boldsymbol{g}^{k}(x)\right]_{i}\right)$. Recalling that the vectors $\boldsymbol{g}^{k}(x)$ form a basis, we may write a priori

$$
\partial_{\ell} \boldsymbol{g}^{q}(x)=-\Gamma_{\ell k}^{q}(x) \boldsymbol{g}^{k}(x),
$$

thereby unambiguously defining functions $\Gamma_{\ell k}^{q}: \Omega \rightarrow \mathbb{R}$. To find their expressions in terms of the mappings $\boldsymbol{\Theta}$ and $\widehat{\boldsymbol{\Theta}}$, we observe that

$$
\Gamma_{\ell k}^{q}(x)=\Gamma_{\ell m}^{q}(x) \delta_{k}^{m}=\Gamma_{\ell m}^{q}(x) \boldsymbol{g}^{m}(x) \cdot \boldsymbol{g}_{k}(x)=-\partial_{\ell} \boldsymbol{g}^{q}(x) \cdot \boldsymbol{g}_{k}(x) .
$$


Hence, noting that $\partial_{\ell}\left(\boldsymbol{g}^{q}(x) \cdot \boldsymbol{g}_{k}(x)\right)=0$ and $\left[\boldsymbol{g}^{q}(x)\right]_{p}=\widehat{\partial}_{p} \widehat{\Theta}^{q}(\widehat{x})$, we obtain

$$
\Gamma_{\ell k}^{q}(x)=\boldsymbol{g}^{q}(x) \cdot \partial_{\ell} \boldsymbol{g}_{k}(x)=\widehat{\partial}_{p} \widehat{\Theta}^{q}(\widehat{x}) \partial_{\ell k} \Theta^{p}(x)=\Gamma_{k \ell}^{q}(x) .
$$

Since $\Theta \in \mathcal{C}^{2}\left(\Omega ; \mathbb{R}^{3}\right)$ and $\widehat{\boldsymbol{\Theta}} \in \mathcal{C}^{1}\left(\widehat{\Omega} ; \mathbb{R}^{3}\right)$ by assumption, the last relations show that $\Gamma_{\ell k}^{q} \in \mathcal{C}^{0}(\Omega)$.

We are now in a position to compute the partial derivatives $\widehat{\partial}_{j} \widehat{v}_{i}(\widehat{x})$ as functions of $x$, by means of the relation $\widehat{v}_{i}(\widehat{x})=v_{k}(x)\left[\boldsymbol{g}^{k}(x)\right]_{i}$. To this end, we first note that a differentiable function $w: \Omega \rightarrow \mathbb{R}$ satisfies

$$
\widehat{\partial}_{j} w(\widehat{\boldsymbol{\Theta}}(\widehat{x}))=\partial_{\ell} w(x) \widehat{\partial}_{j} \widehat{\Theta}^{\ell}(\widehat{x})=\partial_{\ell} w(x)\left[\boldsymbol{g}^{\ell}(x)\right]_{j},
$$

by the chain rule. In particular then,

$$
\begin{aligned}
\widehat{\partial}_{j} \widehat{v}_{i}(\widehat{x}) & =\widehat{\partial}_{j} v_{k}(\widehat{\boldsymbol{\Theta}}(\widehat{x}))\left[\boldsymbol{g}^{k}(x)\right]_{i}+v_{q}(x) \widehat{\partial}_{j}\left[\boldsymbol{g}^{q}(\widehat{\boldsymbol{\Theta}}(\widehat{x}))\right]_{i} \\
& =\partial_{\ell} v_{k}(x)\left[\boldsymbol{g}^{\ell}(x)\right]_{j}\left[\boldsymbol{g}^{k}(x)\right]_{i}+v_{q}(x)\left(\partial_{\ell}\left[\boldsymbol{g}^{q}(x)\right]_{i}\right)\left[\boldsymbol{g}^{\ell}(x)\right]_{j} \\
& =\left(\partial_{\ell} v_{k}(x)-\Gamma_{\ell k}^{q}(x) v_{q}(x)\right)\left[\boldsymbol{g}^{k}(x)\right]_{i}\left[\boldsymbol{g}^{\ell}(x)\right]_{j},
\end{aligned}
$$

since $\partial_{\ell} \boldsymbol{g}^{q}(x)=-\Gamma_{\ell k}^{q}(x) \boldsymbol{g}^{k}(x)$. We have therefore shown that

$$
\widehat{\partial}_{j} \widehat{v}_{i}(\widehat{x})=v_{k \| \ell}(x)\left[\boldsymbol{g}^{k}(x)\right]_{i}\left[\boldsymbol{g}^{\ell}(x)\right]_{j},
$$

where

$$
v_{k \| \ell}(x):=\partial_{\ell} v_{k}(x)-\Gamma_{\ell k}^{q}(x) v_{q}(x)
$$

and $\left[\boldsymbol{g}^{k}(x)\right]_{i}$ and $\Gamma_{\ell k}^{q}(x)$ are defined as above.

(ii) With any vector field $\boldsymbol{v}=v_{i} \boldsymbol{g}^{i}: \Omega \rightarrow \mathbb{R}^{3}$ with $v_{i} \in H^{1}(\Omega)$, we next associate the functions $e_{i j}(\boldsymbol{v}) \in L^{2}(\Omega)$ defined by

$$
e_{i j}(\boldsymbol{v}):=\frac{1}{2}\left(v_{i \| j}+v_{j \| i}\right)=\frac{1}{2}\left(\partial_{j} v_{i}+\partial_{i} v_{j}\right)-\Gamma_{i j}^{p} v_{p}
$$

and, with any vector field $\widehat{\boldsymbol{v}}=\widehat{v}_{i} \widehat{\mathbf{e}}^{i}: \widehat{\Omega} \rightarrow \mathbb{R}^{3}$ with $\widehat{v}_{i} \in H^{1}(\widehat{\Omega})$ we associate the functions $\widehat{e}_{i j}(\widehat{\boldsymbol{v}}) \in L^{2}(\widehat{\Omega})$ defined by

$$
\widehat{e}_{i j}(\widehat{\boldsymbol{v}}):=\frac{1}{2}\left(\widehat{\partial}_{j} \widehat{v}_{i}+\widehat{\partial}_{i} \widehat{v}_{j}\right)
$$

If the fields $\boldsymbol{v}$ and $\widehat{\boldsymbol{v}}$ are related as in (i), it then immediately follows from (i) that

$$
\widehat{e}_{i j}(\widehat{\boldsymbol{v}})(\widehat{x})=\left(e_{k \ell}(\boldsymbol{v})\left[\boldsymbol{g}^{k}\right]_{i}\left[\boldsymbol{g}^{\ell}\right]_{j}\right)(x) \text { for all } \widehat{x}=\boldsymbol{\Theta}(x), x \in \Omega .
$$

(iii) Let a vector field $\boldsymbol{v}=v_{i} \boldsymbol{g}^{i}$ with $v_{i} \in H^{1}(\Omega)$ be such that

$$
e_{i j}(\boldsymbol{v})=0 \text { in } \Omega \text {. }
$$

Then there exist two vectors $\mathbf{a}, \boldsymbol{b} \in \mathbb{R}^{3}$ such that the associated vector field $v_{i} \boldsymbol{g}^{i}$ is of the form

$$
\boldsymbol{v}(x)=\mathbf{a}+\boldsymbol{b} \wedge \boldsymbol{\Theta}(x) \text { for all } x \in \bar{\Omega} .
$$

Next, let $\Gamma_{0}$ be a $\mathrm{d} \Gamma$-measurable subset of the boundary $\partial \Omega$ that satisfies area $\Gamma_{0}>$ 0 , and let a vector field $\boldsymbol{v}=v_{i} \boldsymbol{g}^{i}$ with $v_{i} \in H^{1}(\Omega)$ be such that

$$
e_{i j}(\boldsymbol{v})=0 \text { in } \Omega \text { and } \boldsymbol{v}=\mathbf{0} \text { on } \Gamma_{0} .
$$

Then $\boldsymbol{v}=\mathbf{0}$ in $\Omega$.

It follows from part (ii) that

$$
e_{i j}(\boldsymbol{v})=0 \text { in } \Omega \text { implies } \widehat{e}_{i j}(\widehat{\boldsymbol{v}})=0 \text { in } \widehat{\Omega} .
$$


Then the identity

$$
\widehat{\partial}_{j}\left(\widehat{\partial}_{k} \widehat{v}_{i}\right)=\widehat{\partial}_{j} \widehat{e}_{i k}(\widehat{\boldsymbol{v}})+\widehat{\partial}_{k} \widehat{e}_{i j}(\widehat{\boldsymbol{v}})-\widehat{\partial}_{i} \widehat{e}_{j k}(\widehat{\boldsymbol{v}}) \text { in } \mathcal{D}^{\prime}(\widehat{\Omega})
$$

further shows that

$$
\widehat{e}_{i j}(\widehat{\boldsymbol{v}})=0 \text { in } \widehat{\Omega} \text { implies } \widehat{\partial}_{j}\left(\widehat{\partial}_{k} \widehat{v}_{i}\right)=0 \text { in } \mathcal{D}^{\prime}(\widehat{\Omega}) .
$$

By a classical result from distribution theory (Schwartz [80], p. 60), each function $\widehat{v}_{i}$ is therefore a polynomial of degree $\leq 1$ in the variables $\widehat{x}_{j}$, since the set $\widehat{\Omega}$ is connected. There thus exist constants $a_{i}$ and $b_{i j}$ such that

$$
\widehat{v}_{i}(\widehat{x})=a_{i}+b_{i j} \widehat{x}_{j} \text { for all } \widehat{x}=\left(\widehat{x}_{i}\right) \in \widehat{\Omega} \text {. }
$$

But $\widehat{e}_{i j}(\widehat{\boldsymbol{v}})=0$ also implies that $b_{i j}=-b_{j i}$. Hence there exist two vectors $\mathbf{a}, \boldsymbol{b} \in \mathbb{R}^{3}$ such that ( $\widehat{\boldsymbol{x}}$ denotes the column vector with components $\left.\widehat{x}_{i}\right)$

$$
\widehat{v}_{i}(\widehat{x}) \widehat{\mathbf{e}}^{i}=\mathbf{a}+\boldsymbol{b} \wedge \widehat{\boldsymbol{x}} \text { for all } \widehat{x} \in \widehat{\Omega},
$$

or equivalently, such that

$$
v_{i}(x) \boldsymbol{g}^{i}(x)=\mathbf{a}+\boldsymbol{b} \wedge \boldsymbol{\Theta}(x) \text { for all } x \in \Omega .
$$

Since the set where such a vector field $\widehat{v}_{i} \widehat{\mathrm{e}}^{i}$ vanishes is always of zero area unless $\mathbf{a}=\boldsymbol{b}=\mathbf{0}$ (as is easily proved; see, e.g., Ciarlet [18], Theorem 6.3-4), the assumption area $\Gamma_{0}>0$ implies that $\widehat{\boldsymbol{v}}=\mathbf{0}$.

(iv) We now let

$$
\Omega:=\omega \times\left(-\varepsilon_{0}, \varepsilon_{0}\right),
$$

and we let the mapping $\Theta: \bar{\Omega} \rightarrow \mathbb{R}^{3}$ be defined by

$$
\boldsymbol{\Theta}\left(y, x_{3}\right):=\boldsymbol{\theta}(y)+x_{3} \mathbf{a}_{3}(y) \text { for all } x=\left(x_{i}\right):=\left(y, x_{3}\right) \in \bar{\Omega},
$$

where $\varepsilon_{0}>0$ has been chosen in such a way that the mapping $\Theta$ is a $\mathcal{C}^{2}$-diffeomorphism from $\bar{\Omega}$ into its image $\boldsymbol{\Theta}(\bar{\Omega})$ (Theorem 2.2-1). With any vector field $\boldsymbol{\eta}=\eta_{i} \mathbf{a}^{i}$ with covariant components $\eta_{\alpha}$ in $H^{1}(\omega)$ and $\eta_{3}$ in $H^{2}(\omega)$, let there be associated the vector field $\boldsymbol{v}=v_{i} \boldsymbol{g}^{i}$ defined on $\bar{\Omega}$ by

$$
v_{i}\left(y, x_{3}\right) \boldsymbol{g}^{i}\left(y, x_{3}\right)=\eta_{i}(y) \mathbf{a}^{i}(y)-x_{3}\left(\partial_{\alpha} \eta_{3}+b_{\alpha}^{\sigma} \eta_{\sigma}\right)(y) \mathbf{a}^{\alpha}(y)
$$

for all $\left(y, x_{3}\right) \in \Omega$, where the vectors $\boldsymbol{g}^{i}$ are defined by $\boldsymbol{g}^{i} \cdot \boldsymbol{g}_{j}=\delta_{j}^{i}$.

Then the covariant components $v_{i}$ of the vector field $v_{i} \boldsymbol{g}^{i}$ are in $H^{1}(\Omega)$ and the corresponding functions $e_{i j}(\boldsymbol{v}) \in L^{2}(\Omega)$ defined as in part (ii) are given by

$$
\begin{aligned}
e_{\alpha \beta}(\boldsymbol{v})= & \gamma_{\alpha \beta}(\boldsymbol{\eta})-x_{3} \rho_{\alpha \beta}(\boldsymbol{\eta}) \\
& +\frac{x_{3}^{2}}{2}\left\{b_{\alpha}^{\sigma} \rho_{\beta \sigma}(\boldsymbol{\eta})+b_{\beta}^{\tau} \rho_{\alpha \tau}(\boldsymbol{\eta})-2 b_{\alpha}^{\sigma} b_{\beta}^{\tau} \gamma_{\sigma \tau}(\boldsymbol{\eta})\right\}, \\
e_{i 3}(\boldsymbol{v})= & 0 .
\end{aligned}
$$

Note that, as in the above expressions of the functions $e_{\alpha \beta}(\boldsymbol{v})$, the dependence on $x_{3}$ is explicit, but the dependence with respect to $y \in \bar{\omega}$ is omitted, throughout the proof. The explicit expressions of the functions $\gamma_{\alpha \beta}(\boldsymbol{\eta})$ and $\rho_{\alpha \beta}(\boldsymbol{\eta})$ in terms of the functions $\eta_{i}$ (Theorems 2.8-1 and 2.8-2) are used in this part of the proof.

To prove the above assertion, we proceed in two stages. First, given functions $\eta_{\alpha}, \mathcal{X}_{\alpha} \in H^{1}(\omega)$ and $\eta_{3} \in H^{2}(\omega)$, let the vector field $\boldsymbol{v}=v_{i} \boldsymbol{g}^{i}$ be defined on $\bar{\Omega}$ by

$$
v_{i} \boldsymbol{g}^{i}=\eta_{i} \mathbf{a}^{i}+x_{3} \mathcal{X}_{\alpha} \mathbf{a}^{\alpha} .
$$


Then the functions $v_{i}$ are in $H^{1}(\Omega)$. Besides, the functions $e_{i \| j}(\boldsymbol{v})$ defined as in part (ii) are given by

$$
\begin{aligned}
e_{\alpha \beta}(\boldsymbol{v})= & \left\{\frac{1}{2}\left(\eta_{\alpha \mid \beta}+\eta_{\beta \mid \alpha}\right)-b_{\alpha \beta} \eta_{3}\right\} \\
& +\frac{x_{3}}{2}\left\{\mathcal{X}_{\alpha \mid \beta}+\mathcal{X}_{\beta \mid \alpha}-b_{\alpha}^{\sigma}\left(\eta_{\sigma \mid \beta}-b_{\beta \sigma} \eta_{3}\right)-b_{\beta}^{\tau}\left(\eta_{\tau \mid \alpha}-b_{\alpha \tau} \eta_{3}\right)\right\} \\
& +\frac{x_{3}^{2}}{2}\left\{-b_{\alpha}^{\sigma} \mathcal{X}_{\sigma \mid \beta}-b_{\beta}^{\tau} \mathcal{X}_{\tau \mid \alpha}\right\}, \\
e_{\alpha 3}(\boldsymbol{v})= & \frac{1}{2}\left(\mathcal{X}_{\alpha}+\partial_{\alpha} \eta_{3}+b_{\alpha}^{\sigma} \eta_{\sigma}\right), \\
e_{33}(\boldsymbol{v})= & 0
\end{aligned}
$$

where $\eta_{\alpha \mid \beta}=\partial_{\beta} \eta_{\alpha}-\Gamma_{\alpha \beta}^{\sigma} \eta_{\sigma}$ and $\mathcal{X}_{\alpha \mid \beta}=\partial_{\beta} \mathcal{X}_{\alpha}-\Gamma_{\alpha \beta}^{\sigma} \mathcal{X}_{\sigma}$ designate the covariant derivatives of the fields $\eta_{i} \mathbf{a}^{i}$ and $\mathcal{X}_{i} \mathbf{a}^{i}$ with $\mathcal{X}_{3}=0$.

To see this, we note that

$$
\partial_{\alpha} \mathbf{a}_{3}=-b_{\alpha}^{\sigma} \mathbf{a}_{\sigma}
$$

by the formula of Weingarten (see Section 2.1). Hence, the vectors of the covariant basis associated with the mapping $\boldsymbol{\Theta}=\boldsymbol{\theta}+x_{3} \mathbf{a}_{3}$ are given by

$$
\boldsymbol{g}_{\alpha}=\mathbf{a}_{\alpha}-x_{3} b_{\alpha}^{\sigma} \mathbf{a}_{\sigma} \text { and } \boldsymbol{g}_{3}=\mathbf{a}_{3} .
$$

The assumed regularities of the functions $\eta_{i}$ and $\mathcal{X}_{\alpha}$ imply that

$$
v_{i}=\left(v_{j} \boldsymbol{g}^{j}\right) \cdot \boldsymbol{g}_{i}=\left(\eta_{j} \mathbf{a}^{j}+x_{3} \mathcal{X}_{\alpha} \mathbf{a}^{\alpha}\right) \cdot \boldsymbol{g}_{i} \in H^{1}(\Omega)
$$

since $\boldsymbol{g}_{i} \in \boldsymbol{C}^{1}(\bar{\Omega})$. The announced expressions for the functions $e_{i j}(\boldsymbol{v})$ are then obtained by simple computations, based on the relations $v_{i \| j}=\left\{\partial_{j}\left(v_{k} \boldsymbol{g}^{k}\right)\right\} \cdot \boldsymbol{g}_{i}$ $\left(\right.$ part (i)) and $e_{i j}(\boldsymbol{v})=\frac{1}{2}\left(v_{i \| j}+v_{j \| i}\right)$.

Second, we show that, when

$$
\mathcal{X}_{\alpha}=-\left(\partial_{\alpha} \eta_{3}+b_{\alpha}^{\sigma} \eta_{\sigma}\right),
$$

the functions $e_{i j}(\boldsymbol{v})$ above take the expressions announced in the statement of part (iv).

We first note that $\mathcal{X}_{\alpha} \in H^{1}(\omega)$ (since $b_{\alpha}^{\sigma} \in \mathcal{C}^{1}(\bar{\omega})$ ) and that $e_{\alpha 3}(\boldsymbol{v})=0$ when $\mathcal{X}_{\alpha}=-\left(\partial_{\alpha} \eta_{3}+b_{\alpha}^{\sigma} \eta_{\sigma}\right)$. It thus remains to find the explicit forms of the functions $e_{\alpha \beta}(\boldsymbol{v})$ in this case. Replacing the functions $\mathcal{X}_{\alpha}$ by their expressions and using the symmetry relations $\left.b_{\alpha}^{\sigma}\right|_{\beta}=\left.b_{\beta}^{\sigma}\right|_{\alpha}$ (Theorem 2.8-2), we find that

$$
\begin{array}{r}
\frac{1}{2}\left\{\mathcal{X}_{\alpha \mid \beta}+\mathcal{X}_{\beta \mid \alpha}-b_{\alpha}^{\sigma}\left(\eta_{\sigma \mid \beta}-b_{\beta \sigma} \eta_{3}\right)-b_{\beta}^{\tau}\left(\eta_{\tau \mid \alpha}-b_{\alpha \tau} \eta_{3}\right)\right\} \\
=-\eta_{3 \mid \alpha \beta}-b_{\alpha}^{\sigma} \eta_{\sigma \mid \beta}-b_{\beta}^{\tau} \eta_{\tau \mid \alpha}-b_{\beta \mid \alpha}^{\tau} \eta_{\tau}+b_{\alpha}^{\sigma} b_{\sigma \beta} \eta_{3},
\end{array}
$$

i.e., the factor of $x_{3}$ in $e_{\alpha \beta}(\boldsymbol{v})$ is equal to $-\rho_{\alpha \beta}(\boldsymbol{\eta})$. Finally,

$$
\begin{aligned}
-b_{\alpha}^{\sigma} & \mathcal{X}_{\sigma \mid \beta}-b_{\beta}^{\tau} \mathcal{X}_{\tau \mid \alpha} \\
& =b_{\alpha}^{\sigma}\left(\eta_{3 \mid \beta \sigma}+b_{\sigma \mid \beta}^{\tau} \eta_{\tau}+b_{\sigma}^{\tau} \eta_{\tau \mid \beta}\right)+b_{\beta}^{\tau}\left(\eta_{3 \mid \alpha \tau}+b_{\tau \mid \alpha}^{\sigma} \eta_{\sigma}+b_{\tau}^{\sigma} \eta_{\sigma \mid \alpha}\right) \\
& =b_{\alpha}^{\sigma}\left(\rho_{\beta \sigma}(\boldsymbol{\eta})-b_{\beta}^{\tau} \eta_{\tau \mid \sigma}+b_{\beta}^{\tau} b_{\tau \sigma} \eta_{3}\right)+b_{\beta}^{\tau}\left(\rho_{\alpha \tau}(\boldsymbol{\eta})-b_{\alpha}^{\sigma} \eta_{\sigma \mid \tau}+b_{\alpha}^{\sigma} b_{\sigma \tau} \eta_{3}\right) \\
& =b_{\alpha}^{\sigma} \rho_{\beta \sigma}(\boldsymbol{\eta})+b_{\beta}^{\tau} \rho_{\alpha \tau}(\boldsymbol{\eta})-2 b_{\alpha}^{\sigma} b_{\beta}^{\tau} \gamma_{\sigma \tau}(\boldsymbol{\eta}),
\end{aligned}
$$

i.e., the factor of $\frac{x_{3}^{2}}{2}$ in $e_{\alpha \beta}(\boldsymbol{v})$ is indeed as announced. 
(v) Let the set $\Omega=\omega \times\left(-\varepsilon_{0}, \varepsilon_{0}\right)$ and let the vector field $\boldsymbol{v}=v_{i} \boldsymbol{g}^{i}$ with $v_{i} \in H^{1}(\Omega)$ be defined as in part (iv). By part (iv), the assumption that $\gamma_{\alpha \beta}(\boldsymbol{\eta})=\rho_{\alpha \beta}(\boldsymbol{\eta})=0$ in $\omega$ implies that

$$
e_{i j}(\boldsymbol{v})=0 \text { in } \Omega \text {. }
$$

Therefore, by part (iii), there exist two vectors $\mathbf{a}, \boldsymbol{b} \in \mathbb{R}^{3}$ such that

$$
v_{i}\left(y, x_{3}\right) \boldsymbol{g}^{i}\left(y, x_{3}\right)=\mathbf{a}+\boldsymbol{b} \wedge\left\{\boldsymbol{\theta}(y)+x_{3} \mathbf{a}_{3}(y)\right\} \text { for all }\left(y, x_{3}\right) \in \bar{\Omega} .
$$

Hence

$$
\eta_{i}(y) \mathbf{a}^{i}(y)=\left.v_{i}\left(y, x_{3}\right) \boldsymbol{g}^{i}\left(y, x_{3}\right)\right|_{x_{3}=0}=\mathbf{a}+\boldsymbol{b} \wedge \boldsymbol{\theta}(y) \text { for all } y \in \bar{\omega},
$$

and part (a) of the theorem is established.

Let next $\gamma_{0} \subset \gamma$ be such that length $\gamma_{0}>0$. If in addition $\eta_{i}=\partial_{\nu} \eta_{3}=0$ on $\gamma_{0}$, the functions $\chi_{\alpha}=-\left(\partial_{\alpha} \eta_{3}+b_{\alpha}^{\sigma} \eta_{\sigma}\right)$ vanish on $\gamma_{0}$, since $\eta_{3}=\partial_{\nu} \eta_{3}=0$ on $\gamma_{0}$ implies $\partial_{\alpha} \eta_{3}=0$ on $\gamma_{0}$. Part (iv) then shows that

$$
v_{i}=\left(v_{j} \boldsymbol{g}^{j}\right) \cdot \boldsymbol{g}_{i}=\left(\eta_{j} \mathbf{a}^{j}+x_{3} \mathcal{X}_{\alpha} \mathbf{a}^{\alpha}\right) \cdot \boldsymbol{g}_{i}=0 \text { on } \Gamma_{0}:=\gamma_{0} \times\left[-\varepsilon_{0}, \varepsilon_{0}\right] .
$$

Since area $\Gamma_{0}>0$, part (iii) implies that $\boldsymbol{v}=\mathbf{0}$ in $\bar{\Omega}$, hence that $\boldsymbol{\eta}=\mathbf{0}$ on $\bar{\omega}$ and part (b) of the theorem is established.

We are now in a position to prove the announced Korn's inequality on a surface, "with boundary conditions".

This inequality was first proved by Bernadou \& Ciarlet [11]. It was later given other proofs by Ciarlet \& Miara [33] and Bernadou, Ciarlet \& Miara [12]; then by Akian [4] and Ciarlet \& S. Mardare [32], who showed that it can be directly derived from the three-dimensional Korn inequality in curvilinear coordinates (this idea goes back to Destuynder [45]); then by Blouza \& Le Dret [13], who showed that it still holds under a less stringent smoothness assumption on the mapping $\boldsymbol{\theta}$. We follow here the proof of Bernadou, Ciarlet \& Miara [12].

Theorem 2.9-3. Let $\omega$ be a domain in $\mathbb{R}^{2}$, let $\boldsymbol{\theta} \in \mathcal{C}^{3}\left(\bar{\omega} ; \mathbb{R}^{3}\right)$ be an injective immersion, let $\gamma_{0}$ be a $d \gamma$-measurable subset of $\gamma=\partial \omega$ that satisfies length $\gamma_{0}>0$, and let the space $\boldsymbol{V}(\omega)$ be defined as:

$$
\boldsymbol{V}(\omega):=\left\{\boldsymbol{\eta}=\eta_{i} \mathbf{a}^{i} ; \eta_{\alpha} \in H^{1}(\omega), \eta_{3} \in H^{2}(\omega), \eta_{i}=\partial_{\nu} \eta_{3}=0 \text { on } \gamma_{0}\right\} .
$$

Given $\boldsymbol{\eta}=\eta_{i} \mathbf{a}^{i}$ with $\eta_{\alpha} \in H^{1}(\omega)$ and $\eta_{3} \in H^{2}(\omega)$, let

$$
\begin{aligned}
& \gamma_{\alpha \beta}(\boldsymbol{\eta}):=\left\{\frac{1}{2}\left(\partial_{\beta} \boldsymbol{\eta} \cdot \mathbf{a}_{\alpha}+\partial_{\alpha} \boldsymbol{\eta} \cdot \mathbf{a}_{\beta}\right\} \in L^{2}(\omega),\right. \\
& \rho_{\alpha \beta}(\boldsymbol{\eta}):=\left\{\left(\partial_{\alpha \beta} \boldsymbol{\eta}-\Gamma_{\alpha \beta}^{\sigma} \partial_{\sigma} \boldsymbol{\eta}\right) \cdot \mathbf{a}_{3}\right\} \in L^{2}(\omega)
\end{aligned}
$$

denote the covariant components of the linearized change of metric and linearized change of curvature tensors associated with the displacement field $\boldsymbol{\eta}=\eta_{i} \mathbf{a}^{i}$ of the surface $\boldsymbol{S}=\boldsymbol{\theta}(\bar{\omega})$. Then there exists a constant $c=c\left(\omega, \gamma_{0}, \boldsymbol{\theta}\right)$ such that

$$
\left\{\sum_{\alpha}\left\|\eta_{\alpha}\right\|_{H^{1}(\omega)}^{2}+\left\|\eta_{3}\right\|_{H^{2}(\omega)}^{2}\right\}^{1 / 2} \leq c\left\{\sum_{\alpha, \beta}\left\|\gamma_{\alpha \beta}(\boldsymbol{\eta})\right\|_{L^{2}(\omega)}^{2}+\sum_{\alpha, \beta}\left\|\rho_{\alpha \beta}(\boldsymbol{\eta})\right\|_{L^{2}(\omega)}^{2}\right\}^{1 / 2}
$$

for all $\boldsymbol{\eta}=\eta_{i} \mathbf{a}^{i} \in \boldsymbol{V}(\omega)$. 
Proof. Let the space $\widetilde{\boldsymbol{V}}(\omega):=\left\{\boldsymbol{\eta}=\eta_{i} \mathbf{a}^{i} ; \eta_{\alpha} \in H^{1}(\omega), \eta_{3} \in H^{2}(\omega)\right\}$ be equipped with the norm

$$
\|\boldsymbol{\eta}\|_{H^{1}(\omega) \times H^{1}(\omega) \times H^{2}(\omega)}:=\left\{\sum_{\alpha}\left\|\eta_{\alpha}\right\|_{H^{1}(\omega)}^{2}+\left\|\eta_{3}\right\|_{H^{2}(\omega)}^{2}\right\}^{1 / 2} .
$$

If the announced inequality is false, there exists a sequence $\left(\boldsymbol{\eta}^{k}\right)_{k=1}^{\infty}$ of vector fields $\boldsymbol{\eta}^{k} \in \boldsymbol{V}(\omega)$ such that

$$
\begin{gathered}
\left\|\boldsymbol{\eta}^{k}\right\|_{H^{1}(\omega) \times H^{1}(\omega) \times H^{2}(\omega)}=1 \text { for all } k, \\
\lim _{k \rightarrow \infty}\left\{\sum_{\alpha, \beta}\left\|\gamma_{\alpha \beta}\left(\boldsymbol{\eta}^{k}\right)\right\|_{L^{2}(\omega)}^{2}+\sum_{\alpha, \beta}\left\|\rho_{\alpha \beta}\left(\boldsymbol{\eta}^{k}\right)\right\|_{L^{2}(\omega)}^{2}\right\}^{1 / 2}=0 .
\end{gathered}
$$

Since the sequence $\left(\boldsymbol{\eta}^{k}\right)_{k=1}^{\infty}$ is bounded in $\tilde{\boldsymbol{V}}(\omega)$, a subsequence $\left(\boldsymbol{\eta}^{\sigma(k)}\right)_{k=1}^{\infty}(\sigma$ : $\mathbb{N} \rightarrow \mathbb{N}$ is an increasing function) converges in $\widetilde{\boldsymbol{V}}(\omega)$ by the Rellich-Kondrašov theorem. Furthermore, each sequence $\left(\gamma_{\alpha \beta}\left(\boldsymbol{\eta}^{\sigma(k)}\right)\right)_{k=1}^{\infty}$ and $\left(\rho_{\alpha \beta}\left(\boldsymbol{\eta}^{\sigma(k)}\right)\right)_{k=1}^{\infty}$ also converges in $L^{2}(\omega)$ (to 0 , but this information is not used at this stage) since

$$
\lim _{k \rightarrow \infty}\left\{\sum_{\alpha, \beta}\left\|\gamma_{\alpha \beta}\left(\boldsymbol{\eta}^{\sigma(k)}\right)\right\|_{L^{2}(\omega)}^{2}+\sum_{\alpha, \beta}\left\|\rho_{\alpha \beta}\left(\boldsymbol{\eta}^{\sigma(k)}\right)\right\|_{L^{2}(\omega)}^{2}\right\}^{1 / 2}=0
$$

The subsequence $\left(\boldsymbol{\eta}^{\sigma(k)}\right)_{k=1}^{\infty}$ is thus a Cauchy sequence with respect to the norm

$$
\boldsymbol{\eta}=\eta_{i} \mathbf{a}^{i} \rightarrow\left\{\sum_{\alpha}\left\|\eta_{\alpha}\right\|_{L^{2}(\omega)}^{2}+\left\|\eta_{3}\right\|_{H^{1}(\omega)}^{2}+\sum_{\alpha, \beta}\left\|\gamma_{\alpha \beta}(\boldsymbol{\eta})\right\|_{L^{2}(\omega)}^{2}+\sum_{\alpha, \beta}\left|\rho_{\alpha \beta}(\boldsymbol{\eta})\right|_{L^{2}(\omega)}^{2}\right\}^{1 / 2},
$$

hence with respect to the norm $\|\cdot\|_{H^{1}(\omega) \times H^{1}(\omega) \times H^{2}(\omega)}$ by Korn's inequality without boundary conditions (Theorem 2.9-1).

The space $\boldsymbol{V}(\omega)$ being complete as a closed subspace of the space $\tilde{\boldsymbol{V}}(\omega)$, there exists $\boldsymbol{\eta} \in \boldsymbol{V}(\omega)$ such that

$$
\boldsymbol{\eta}^{\sigma(k)} \rightarrow \boldsymbol{\eta} \text { in } \widetilde{\boldsymbol{V}}(\omega)
$$

and the limit $\boldsymbol{\eta}$ satisfies

$$
\begin{aligned}
& \left\|\gamma_{\alpha \beta}(\boldsymbol{\eta})\right\|_{L^{2}(\omega)}=\lim _{k \rightarrow \infty}\left\|\gamma_{\alpha \beta}\left(\boldsymbol{\eta}^{\sigma(k)}\right)\right\|_{L^{2}(\omega)}=0, \\
& \left\|\rho_{\alpha \beta}(\boldsymbol{\eta})\right\|_{L^{2}(\omega)}=\lim _{k \rightarrow \infty}\left\|\rho_{\alpha \beta}\left(\boldsymbol{\eta}^{\sigma(k)}\right)\right\|_{L^{2}(\omega)}=0 .
\end{aligned}
$$

Hence $\boldsymbol{\eta}=\mathbf{0}$ by Theorem 2.9-2. But this contradicts the relations

$$
\left\|\boldsymbol{\eta}^{\sigma(k)}\right\|_{H^{1}(\omega) \times H^{1}(\omega) \times H^{2}(\omega)}=1 \text { for all } k \geq 1,
$$

and the proof is complete.

If the mapping $\boldsymbol{\theta}$ is of the form $\boldsymbol{\theta}\left(y_{1}, y_{2}\right)=\left(y_{1}, y_{2}, 0\right)$ for all $\left(y_{1}, y_{2}\right) \in \overline{\boldsymbol{\omega}}$, the inequality of Theorem 2.9-3 reduces to two distinct inequalities (obtained by letting first $\eta_{\alpha}=0$, then $\eta_{3}=0$ ):

$$
\left\|\eta_{3}\right\|_{H^{2}(\omega)} \leq c\left\{\sum_{\alpha, \beta}\left\|\partial_{\alpha \beta} \eta_{3}\right\|_{L^{2}(\omega)}^{2}\right\}^{1 / 2}
$$

for all $\eta_{3} \in H^{2}(\omega)$ satisfying $\eta_{3}=\partial_{\nu} \eta_{3}=0$ on $\gamma_{0}$, and

$$
\left\{\sum_{\alpha}\left\|\eta_{\alpha}\right\|_{H^{1}(\omega)}^{2}\right\}^{1 / 2} \leq c\left\{\sum_{\alpha, \beta}\left\|\frac{1}{2}\left(\partial_{\beta} \eta_{\alpha}+\partial_{\alpha} \eta_{\beta}\right)\right\|_{L^{2}(\omega)}^{2}\right\}^{1 / 2}
$$


for all $\eta_{\alpha} \in H^{1}(\omega)$ satisfying $\eta_{\alpha}=0$ on $\gamma_{0}$. The first inequality is a well-known property of Sobolev spaces. The second inequality is the two-dimensional Korn inequality in Cartesian coordinates. Both play a central rôle in the existence theory for linear two-dimensional plate equations (see, e.g., Ciarlet [19], Theorems 1.5-1 and 1.5-2).

As shown by Blouza \& Le Dret [13], Le Dret [61], and Anicic, Le Dret \& Raoult [7], the regularity assumptions made on the mapping $\boldsymbol{\theta}$ and on the field $\boldsymbol{\eta}$ in both the infinitesimal rigid displacement lemma and the Korn inequality on a surface of Theorems 2.9-2 and 2.9-3 can be substantially weakened.

It is remarkable that, for specific geometries and boundary conditions, a Korn inequality can be established that only involves the linearized change of metric tensors. More specifically, Ciarlet \& Lods [27] and Ciarlet \& Sanchez-Palencia [35] have established the following Korn inequality "on an elliptic surface":

Let $\omega$ be a domain in $\mathbb{R}^{2}$ and let $\boldsymbol{\theta} \in \mathcal{C}^{2,1}\left(\bar{\omega} ; \mathbb{R}^{3}\right)$ be an injective immersion with the property that the surface $S=\boldsymbol{\theta}(\bar{\omega})$ is elliptic, in the sense that all its points are elliptic (this means that the Gaussian curvature is $>0$ everywhere on $S$ ). Then there exists a constant $c_{M}=c_{M}(\omega, \boldsymbol{\theta})>0$ such that

$$
\left\{\sum_{\alpha}\left\|\eta_{\alpha}\right\|_{H^{1}(\omega)}^{2}+\left\|\eta_{3}\right\|_{L^{2}(\omega}^{2}\right\}^{1 / 2} \leq c_{M}\left\{\sum_{\alpha, \beta}\left\|\gamma_{\alpha \beta}(\boldsymbol{\eta})\right\|_{L^{2}(\omega)}^{2}\right\}^{1 / 2}
$$

for all $\boldsymbol{\eta}=\eta_{i} \mathbf{a}^{i}$ with $\eta_{\alpha} \in H_{0}^{1}(\omega)$ and $\eta_{3} \in L^{2}(\omega)$.

Remarks. (1) The norm $\left\|\eta_{3}\right\|_{H^{2}(\omega)}$ appearing in the left-hand side of the Korn inequality on a "general" surface (Theorem 2.9-3) is now replaced by the norm $\left\|\eta_{3}\right\|_{L^{2}(\omega)}$. This replacement reflects that it is enough that $\eta_{\alpha} \in H^{1}(\omega)$ and $\eta_{3} \in$ $L^{2}(\omega)$ in order that $\gamma_{\alpha \beta}(\boldsymbol{\eta}) \in L^{2}(\omega)$, where $\boldsymbol{\eta}=\eta_{i} \mathbf{a}^{i}$. As a result, no boundary condition can be imposed on $\eta_{3}$.

(2) The Korn inequality on an elliptic surface was first established by Destuynder [45], Theorems 6.1 and 6.5, under the additional assumption that the $\mathcal{C}^{0}(\bar{\omega})$-norms of the Christoffel symbols are small enough.

Only compact surfaces defined by a single injective immersion $\boldsymbol{\theta} \in \mathcal{C}^{3}(\bar{\omega})$ have been considered so far. By contrast, a compact surface $S$ "without boundary" (such as an ellipsoid or a torus) is defined by means of a finite number $I \geq 2$ of injective immersions $\boldsymbol{\theta}_{i} \in \mathcal{C}^{3}\left(\bar{\omega}_{i}\right), 1 \leq i \leq I$, where the sets $\omega_{i}$ are domains in $\mathbb{R}^{2}$, in such a way that $S=\bigcup_{i \in I} \boldsymbol{\theta}_{i}\left(\omega_{i}\right)$. As shown by S. Mardare [67], the Korn inequality "without boundary conditions" (Theorem 2.9-1) and the infinitesimal rigid displacement lemma on a surface "without boundary conditions" (Theorem 2.9-2) can be both extended to such surfaces without boundary.

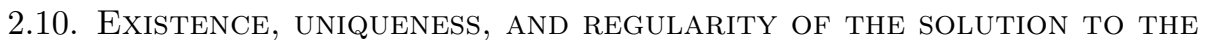 LINEAR KOITER SHELL MODEL}

Let $\omega$ be a domain in $\mathbb{R}^{2}$, let $\gamma_{0}$ be a measurable subset of $\gamma=\partial \omega$ that satisfies length $\gamma_{0}>0$, let $\partial_{\nu}$ denote the outer normal derivative operator along $\partial \omega$, let $\boldsymbol{\theta} \in \mathcal{C}^{3}\left(\bar{\omega} ; \mathbb{R}^{3}\right)$ be an immersion, and let the space $\boldsymbol{V}(\omega)$ be defined by

$$
\boldsymbol{V}(\omega):=\left\{\boldsymbol{\eta}=\eta_{i} \mathbf{a}^{i} ; \eta_{\alpha} \in H^{1}(\omega), \eta_{3} \in H^{2}(\omega) ; \eta_{i}=\partial_{\nu} \eta_{3}=0 \text { on } \gamma_{0}\right\},
$$


where $\gamma_{0}$ is a $\mathrm{d} \gamma$-measurable subset of $\gamma:=\partial \omega$ that satisfies length $\gamma_{0}>0$. Our primary objective consists in showing that the bilinear form $B: \boldsymbol{V}(\omega) \times \boldsymbol{V}(\omega) \rightarrow \mathbb{R}$ defined by

$$
B(\boldsymbol{\zeta}, \boldsymbol{\eta}):=\int_{\omega}\left\{\varepsilon a^{\alpha \beta \sigma \tau} \gamma_{\sigma \tau}(\boldsymbol{\zeta}) \gamma_{\alpha \beta}(\boldsymbol{\eta})+\frac{\varepsilon^{3}}{3} a^{\alpha \beta \sigma \tau} \rho_{\sigma \tau}(\boldsymbol{\zeta}) \rho_{\alpha \beta}(\boldsymbol{\eta})\right\} \sqrt{a} d y
$$

for all $(\boldsymbol{\zeta}, \boldsymbol{\eta}) \in \boldsymbol{V}(\omega) \times \boldsymbol{V}(\omega)$ is $\boldsymbol{V}(\omega)$-elliptic.

As a preliminary, we establish the uniform positive-definiteness of the elasticity tensor of the shell, given here by means of its contravariant components $a^{\alpha \beta \sigma \tau}$ (note that the assumptions on the Lamé constants, viz., $3 \lambda+2 \mu>0$ and $\mu>0$, are weaker than those usually made for elastic materials).

Theorem 2.10-1. Let $\omega$ be a domain in $\mathbb{R}^{2}$, let $\boldsymbol{\theta} \in \mathcal{C}^{3}\left(\bar{\omega} ; \mathbb{R}^{3}\right)$ be an injective immersion, let $a^{\alpha \beta}$ denote the contravariant components of the metric tensor of the surface $\boldsymbol{\theta}(\bar{\omega})$, let the contravariant components of the two-dimensional elasticity tensor of the shell be given by

$$
a^{\alpha \beta \sigma \tau}=\frac{4 \lambda \mu}{\lambda+2 \mu} a^{\alpha \beta} a^{\sigma \tau}+2 \mu\left(a^{\alpha \sigma} a^{\beta \tau}+a^{\alpha \tau} a^{\beta \sigma}\right),
$$

and assume that $3 \lambda+2 \mu>0$ and $\mu>0$. Then there exists a constant $c_{e}=$ $c_{e}(\omega, \boldsymbol{\theta}, \lambda, \mu)>0$ such that

$$
\sum_{\alpha, \beta}\left|t_{\alpha \beta}\right|^{2} \leq c_{e} a^{\alpha \beta \sigma \tau}(y) t_{\sigma \tau} t_{\alpha \beta}
$$

for all $y \in \bar{\omega}$ and all symmetric matrices $\left(t_{\alpha \beta}\right)$.

Proof. In what follows, $\mathbb{M}^{2}$ and $\mathbb{S}^{2}$ respectively designate the set of all real matrices of order two and the set of all real symmetric matrices of order two.

(i) To begin with, we establish a crucial inequality. Let $\chi$ and $\mu$ be two constants satisfying $\chi+\mu>0$ and $\mu>0$. Then there exists a constant $\gamma=\gamma(\chi, \mu)>0$ such that

$$
\gamma \operatorname{tr}\left(\boldsymbol{B}^{T} \boldsymbol{B}\right) \leq \chi(\operatorname{tr} \boldsymbol{B})^{2}+2 \mu \operatorname{tr}\left(\boldsymbol{B}^{T} \boldsymbol{B}\right) \text { for all } \boldsymbol{B} \in \mathbb{M}^{2} .
$$

If $\chi \geq 0$ and $\mu>0$, this inequality holds with $\gamma=2 \mu$. It thus remains to consider the case where $-\mu<\chi<0$ and $\mu>0$. Given any matrix $\boldsymbol{B} \in \mathbb{M}^{2}$, define the matrix $C \in \mathbb{M}^{2}$ by

$$
\boldsymbol{C}=\mathbb{A} \boldsymbol{B}:=\chi(\operatorname{tr} \boldsymbol{B}) \boldsymbol{I}+2 \mu \boldsymbol{B} .
$$

The linear mapping $\mathbb{A}: \mathbb{M}^{2} \rightarrow \mathbb{M}^{2}$ defined in this fashion can be easily inverted if $\chi+\mu \neq 0$ and $\mu \neq 0$, as

$$
\boldsymbol{B}=\mathbb{A}^{-1} \boldsymbol{C}=-\frac{\chi}{4 \mu(\chi+\mu)}(\operatorname{tr} \boldsymbol{C}) \boldsymbol{I}+\frac{1}{2 \mu} \boldsymbol{C} .
$$

Noting that the bilinear mapping

$$
(\boldsymbol{B}, \boldsymbol{C}) \in \mathbb{M}^{2} \times \mathbb{M}^{2} \rightarrow \boldsymbol{B}: \boldsymbol{C}:=\operatorname{tr}\left(\boldsymbol{B}^{T} \boldsymbol{C}\right)
$$

defines an inner product over the space $\mathbb{M}^{2}$, we thus obtain

$$
\begin{aligned}
\chi(\operatorname{tr} \boldsymbol{B})^{2}+2 \mu \operatorname{tr}\left(\boldsymbol{B}^{T} \boldsymbol{B}\right) & =(\mathbb{A} \boldsymbol{B}): \boldsymbol{B}=\boldsymbol{C}: \mathbb{A}^{-1} \boldsymbol{C} \\
& =-\frac{\chi}{4 \mu(\chi+\mu)}(\operatorname{tr} \boldsymbol{C})^{2}+\frac{1}{2 \mu} \operatorname{tr}\left(\boldsymbol{C}^{T} \boldsymbol{C}\right) \geq \frac{1}{2 \mu} \boldsymbol{C}: \boldsymbol{C}
\end{aligned}
$$


for any $\boldsymbol{B}=\mathbb{A}^{-1} \boldsymbol{C} \in \mathbb{M}^{2}$ if $-\mu<\chi<0$ and $\mu>0$. Since there clearly exists a constant $\beta=\beta(\chi, \mu)>0$ such that

$$
\boldsymbol{B}: \boldsymbol{B} \leq \beta \boldsymbol{C}: \boldsymbol{C} \text { for all } \boldsymbol{B}=\mathbb{A}^{-1} \boldsymbol{C} \in \mathbb{M}^{2},
$$

the announced inequality also holds if $-\mu<\chi<0$ and $\mu>0$, with $\gamma=(2 \mu \beta)^{-1}$ in this case.

(ii) We next show that, for any $y \in \bar{\omega}$ and any nonzero symmetric matrix $\left(t_{\alpha \beta}\right)$,

$$
a^{\alpha \beta \sigma \tau}(y) t_{\sigma \tau} t_{\alpha \beta} \geq \gamma a^{\alpha \sigma}(y) a^{\beta \tau}(y) t_{\sigma \tau} t_{\alpha \beta}>0,
$$

where $\gamma=\gamma(\chi, \mu)>0$ is the constant found in (i).

Given any $y \in \bar{\omega}$ and any symmetric matrix $\left(t_{\alpha \beta}\right)$, let

$$
\boldsymbol{A}(y)=\left(a^{\alpha \beta}(y)\right) \text { and } \boldsymbol{T}=\left(t_{\alpha \beta}\right),
$$

let $\boldsymbol{K}(y) \in \mathbb{S}^{2}$ be the unique square root of $\boldsymbol{A}(y)$ (i.e., the unique positive-definite symmetric matrix that satisfies $\left.(\boldsymbol{K}(y))^{2}=\boldsymbol{A}(y)\right)$, and let

$$
\boldsymbol{B}(y):=\boldsymbol{K}(y) \boldsymbol{T} \boldsymbol{K}(y) \in \mathbb{S}^{2} .
$$

Then

$$
\frac{1}{2} a^{\alpha \beta \sigma \tau}(y) t_{\sigma \tau} t_{\alpha \beta}=\chi(\operatorname{tr} \boldsymbol{B}(y))^{2}+2 \mu \operatorname{tr}\left(\boldsymbol{B}(y)^{T} \boldsymbol{B}(y)\right) \text { with } \chi:=\frac{2 \lambda \mu}{\lambda+2 \mu} .
$$

By the inequality established in part (i), there thus exists a constant $\alpha(\lambda, \mu)>0$ such that

$$
\frac{1}{2} a^{\alpha \beta \sigma \tau}(y) t_{\sigma \tau} t_{\alpha \beta} \geq \alpha \operatorname{tr}\left(\boldsymbol{B}(y)^{T} \boldsymbol{B}(y)\right)
$$

if $\chi+\mu>0$ and $\mu>0$, or equivalently, if $3 \lambda+2 \mu>0$ and $\mu>0$.

(iii) Conclusion: Since the mapping

$$
\left(y,\left(t_{\alpha \beta}\right)\right) \in \boldsymbol{K}:=\bar{\omega} \times\left\{\left(t_{\alpha \beta}\right) \in \mathbb{S}^{2} ; \sum_{\alpha, \beta}\left|t_{\alpha \beta}\right|^{2}=1\right\} \longrightarrow a^{\alpha \sigma}(y) a^{\beta \tau}(y) t_{\sigma \tau} t_{\alpha \beta},
$$

is continuous and its domain of definition is compact, we infer that

$$
\delta=\delta(\omega ; \boldsymbol{\theta}):=\inf _{\left(y,\left(t_{\alpha \beta}\right)\right) \in \boldsymbol{K}} a^{\alpha \sigma}(y) a^{\beta \tau}(y) t_{\sigma \tau} t_{\alpha \beta}>0 .
$$

Hence

$$
\delta \sum_{\alpha, \beta}\left|t_{\alpha \beta}\right|^{2} \leq a^{\alpha \sigma}(y) a^{\beta \tau}(y) t_{\sigma \tau} t_{\alpha \beta}
$$

and thus

$$
\sum_{\alpha, \beta}\left|t_{\alpha \beta}\right|^{2} \leq c_{e} a^{\alpha \beta \sigma \tau}(y) t_{\sigma \tau} t_{\alpha \beta}
$$

for all $y \in \bar{\omega}$ and all symmetric matrices $\left(t_{\alpha \beta}\right)$, with $c_{e}:=(\gamma \delta)^{-1}$.

Combined with Korn's inequality "with boundary conditions" (Theorem 2.93 ), the positive definiteness of the elasticity tensor leads to the existence of a weak solution, i.e., a solution to the variational equations of the linear Koiter shell model. 
Theorem 2.10-2. Let $\omega$ be a domain in $\mathbb{R}^{2}$, let $\gamma_{0}$ be a subset of $\gamma=\partial \omega$ with length $\gamma_{0}>0$, and let $\boldsymbol{\theta} \in \mathcal{C}^{3}\left(\bar{\omega} ; \mathbb{R}^{3}\right)$ be an injective immersion. Finally, let there be given constants $\lambda$ and $\mu$ that satisfy $3 \lambda+2 \mu>0$ and $\mu>0$, and functions $p^{\alpha, \varepsilon} \in L^{r}(\omega)$ for some $r>1$ and $p^{3, \varepsilon} \in L^{1}(\omega)$.

Then there is one and only one solution $\boldsymbol{\zeta}^{\varepsilon}=\zeta_{i}^{\varepsilon} \mathbf{a}^{i}$ to the variational problem:

$$
\begin{gathered}
\boldsymbol{\zeta}^{\varepsilon} \in \boldsymbol{V}(\omega)=\left\{\boldsymbol{\eta}=\eta_{i} \mathbf{a}^{i} ; \eta_{\alpha} \in H^{1}(\omega), \eta_{3} \in H^{2}(\omega), \eta_{i}=\partial_{\nu} \eta_{3}=0 \text { on } \gamma_{0}\right\}, \\
\int_{\omega}\left\{\varepsilon a^{\alpha \beta \sigma \tau} \gamma_{\sigma \tau}\left(\boldsymbol{\zeta}^{\varepsilon}\right) \gamma_{\alpha \beta}(\boldsymbol{\eta})+\frac{\varepsilon^{3}}{3} a^{\alpha \beta \sigma \tau} \rho_{\sigma \tau}\left(\boldsymbol{\zeta}^{\varepsilon}\right) \rho_{\alpha \beta}(\boldsymbol{\eta})\right\} \sqrt{a} d y \\
=\int_{\omega} p^{i, \varepsilon} \eta_{i} \sqrt{a} d y \text { for all } \boldsymbol{\eta}=\eta_{i} \mathbf{a}^{i} \in \boldsymbol{V}(\omega),
\end{gathered}
$$

where

$$
\begin{aligned}
a^{\alpha \beta \sigma \tau} & =\frac{4 \lambda \mu}{\lambda+2 \mu} a^{\alpha \beta} a^{\sigma \tau}+2 \mu\left(a^{\alpha \sigma} a^{\beta \tau}+a^{\alpha \tau} a^{\beta \sigma}\right), \\
\gamma_{\alpha \beta}(\boldsymbol{\eta}) & =\frac{1}{2}\left(\partial_{\beta} \boldsymbol{\eta} \cdot \mathbf{a}_{\alpha}+\partial_{\alpha} \boldsymbol{\eta} \cdot \mathbf{a}_{\beta}\right) \text { and } \rho_{\alpha \beta}(\boldsymbol{\eta})=\left(\partial_{\alpha \beta} \boldsymbol{\eta}-\Gamma_{\alpha \beta}^{\sigma} \partial_{\sigma} \boldsymbol{\eta}\right) \cdot \mathbf{a}_{3} .
\end{aligned}
$$

The field $\boldsymbol{\zeta}^{\varepsilon} \in \boldsymbol{V}(\omega)$ is also the unique solution to the minimization problem:

$$
j\left(\boldsymbol{\zeta}^{\varepsilon}\right)=\inf _{\boldsymbol{\eta} \in \boldsymbol{V}(\omega)} j(\boldsymbol{\eta}),
$$

where

$$
\begin{aligned}
j(\boldsymbol{\eta}):= & \frac{1}{2} \int_{\omega}\left\{\varepsilon a^{\alpha \beta \sigma \tau} \gamma_{\sigma \tau}(\boldsymbol{\eta}) \gamma_{\alpha \beta}(\boldsymbol{\eta})+\frac{\varepsilon^{3}}{3} a^{\alpha \beta \sigma \tau} \rho_{\sigma \tau}(\boldsymbol{\eta}) \rho_{\alpha \beta}(\boldsymbol{\eta})\right\} \sqrt{a} d y \\
& -\int_{\omega} p^{i, \varepsilon} \eta_{i} \sqrt{a} d y .
\end{aligned}
$$

Proof. As a closed subspace of the space $\tilde{\boldsymbol{V}}(\omega):=\left\{\boldsymbol{\eta}=\eta_{i} \mathbf{a}^{i} ; \eta_{\alpha} \in H^{1}(\omega), \eta_{3} \in\right.$ $\left.H^{2}(\omega)\right\}$ equipped with the hilbertian norm

$$
\|\boldsymbol{\eta}\|_{H^{1}(\omega) \times H^{1}(\omega) \times H^{2}(\omega)}:=\left\{\sum_{\alpha}\left\|\eta_{\alpha}\right\|_{H^{1}(\omega)}^{2}+\left\|\eta_{3}\right\|_{H^{2}(\omega)}^{2}\right\}^{1 / 2},
$$

the space $\boldsymbol{V}(\omega)$ is a Hilbert space. The assumptions made on the mapping $\boldsymbol{\theta}$ ensure in particular that the vector fields $\mathbf{a}_{i}$ and $\mathbf{a}^{i}$ belong to $\mathcal{C}^{2}\left(\bar{\omega} ; \mathbb{R}^{3}\right)$ and that the functions $a^{\alpha \beta \sigma \tau}, \Gamma_{\alpha \beta}^{\sigma}$, and $a$ are continuous on the compact set $\bar{\omega}$. Hence the bilinear form defined by the left-hand side of the variational equations is continuous over the space $\tilde{\boldsymbol{V}}(\omega)$.

The continuous embeddings of the space $H^{1}(\omega)$ into the space $L^{s}(\omega)$ for any $s \geq 1$ and of the space $H^{2}(\omega)$ into the space $\mathcal{C}^{0}(\bar{\omega})$ show that the linear form defined by the right-hand side is continuous over the same space.

Since the symmetric matrix $\left(a_{\alpha \beta}(y)\right)$ is positive-definite for all $y \in \bar{\omega}$, there exists $a_{0}$ such that $a(y) \geq a_{0}>0$ for all $y \in \bar{\omega}$.

Finally, the Korn inequality "with boundary conditions" (Theorem 2.9-3) and the uniform positive definiteness of the elasticity tensor of the shell (Theorem 2.101) together imply that

$$
\begin{aligned}
& \min \left\{\varepsilon, \frac{\varepsilon^{3}}{3}\right\} c_{e}^{-1} c^{-2} \sqrt{a_{0}}\left(\sum_{\alpha}\left\|\eta_{\alpha}\right\|_{H^{1}(\omega)}^{2}+\left\|\eta_{3}\right\|_{H^{2}(\omega)}^{2}\right) \\
& \leq \int_{\omega}\left\{\varepsilon a^{\alpha \beta \sigma \tau} \gamma_{\sigma \tau}(\boldsymbol{\eta}) \gamma_{\alpha \beta}(\boldsymbol{\eta})+\frac{\varepsilon^{3}}{3} a^{\alpha \beta \sigma \tau} \rho_{\sigma \tau}(\boldsymbol{\eta}) \rho_{\alpha \beta}(\boldsymbol{\eta})\right\} \sqrt{a} d y
\end{aligned}
$$


for all $\boldsymbol{\eta}=\eta_{i} \mathbf{a}^{i} \in \boldsymbol{V}(\omega)$. Hence the bilinear form $B$ is $\boldsymbol{V}(\omega)$-elliptic.

The Lax-Milgram lemma then shows that the variational equations have one and only one solution. Since the bilinear form is symmetric, this solution is also the unique solution of the minimization problem stated in the theorem.

The above existence and uniqueness result applies to linearized pure displacement and displacement-traction problems, i.e., those that correspond to length $\gamma_{0}>0$.

We next derive the boundary value problem that is, at least formally, equivalent to the variational equations of Theorem 2.10-2. In what follows, $\gamma_{1}:=\gamma \backslash \gamma_{0},\left(\nu_{\alpha}\right)$ is the unit outer normal vector along $\gamma, \tau_{1}:=-\nu_{2}, \tau_{2}:=\nu_{1}$, and $\partial_{\tau} \chi:=\tau_{\alpha} \partial_{\alpha} \chi$ denotes the tangential derivative of $\chi$ in the direction of the vector $\left(\tau_{\alpha}\right)$.

Theorem 2.10-3. Let $\omega$ be a domain in $\mathbb{R}^{2}$ and let $\boldsymbol{\theta} \in \mathcal{C}^{3}\left(\bar{\omega} ; \mathbb{R}^{3}\right)$ be an injective immersion. Assume that the boundary $\gamma$ of $\omega$ and the functions $p^{i, \varepsilon}$ are smooth enough. If the solution $\boldsymbol{\zeta}^{\varepsilon}=\zeta_{i}^{\varepsilon} \mathbf{a}^{i}$ to the variational equations found in Theorem 2.10-2 is smooth enough, then $\boldsymbol{\zeta}^{\varepsilon}$ is also a solution to the following boundary value problem:

$$
\begin{aligned}
\left.m^{\alpha \beta}\right|_{\alpha \beta}-b_{\alpha}^{\sigma} b_{\sigma \beta} m^{\alpha \beta}-b_{\alpha \beta} n^{\alpha \beta} & =p^{3, \varepsilon} \text { in } \omega, \\
-\left.\left(n^{\alpha \beta}+b_{\sigma}^{\alpha} m^{\sigma \beta}\right)\right|_{\beta}-b_{\sigma}^{\alpha}\left(\left.m^{\sigma \beta}\right|_{\beta}\right) & =p^{\alpha, \varepsilon} \text { in } \omega, \\
\zeta_{i}^{\varepsilon}=\partial_{\nu} \zeta_{3}^{\varepsilon} & =0 \text { on } \gamma_{0}, \\
m^{\alpha \beta} \nu_{\alpha} \nu_{\beta} & =0 \text { on } \gamma_{1}, \\
\left(\left.m^{\alpha \beta}\right|_{\alpha}\right) \nu_{\beta}+\partial_{\tau}\left(m^{\alpha \beta} \nu_{\alpha} \tau_{\beta}\right) & =0 \text { on } \gamma_{1}, \\
\left(n^{\alpha \beta}+2 b_{\sigma}^{\alpha} m^{\sigma \beta}\right) \nu_{\beta} & =0 \text { on } \gamma_{1},
\end{aligned}
$$

where

$$
n^{\alpha \beta}:=\varepsilon a^{\alpha \beta \sigma \tau} \gamma_{\sigma \tau}\left(\boldsymbol{\zeta}^{\varepsilon}\right) \text { and } m^{\alpha \beta}:=\frac{\varepsilon^{3}}{3} a^{\alpha \beta \sigma \tau} \rho_{\sigma \tau}\left(\boldsymbol{\zeta}^{\varepsilon}\right),
$$

and, for an arbitrary tensor field with smooth enough covariant components $t^{\alpha \beta}$ : $\bar{\omega} \rightarrow \mathbb{R}$

$$
\begin{aligned}
\left.t^{\alpha \beta}\right|_{\beta} & :=\partial_{\beta} t^{\alpha \beta}+\Gamma_{\beta \sigma}^{\alpha} t^{\beta \sigma}+\Gamma_{\beta \sigma}^{\beta} t^{\alpha \sigma}, \\
\left.t^{\alpha \beta}\right|_{\alpha \beta} & :=\partial_{\alpha}\left(\left.t^{\alpha \beta}\right|_{\beta}\right)+\Gamma_{\alpha \sigma}^{\sigma}\left(\left.t^{\alpha \beta}\right|_{\beta}\right) .
\end{aligned}
$$

Proof. For simplicity, we give the proof only in the case where $\gamma_{0}=\gamma$, i.e., when the space $\boldsymbol{V}(\omega)$ of Theorem 2.10-2 reduces to

$$
\boldsymbol{V}(\omega)=\left\{\boldsymbol{\eta}=\eta_{i} \mathbf{a}^{i} ; \eta_{\alpha} \in H_{0}^{1}(\omega), \eta_{3} \in H_{0}^{2}(\omega)\right\} .
$$

The extension to the case where length $\gamma_{1}>0$ is straightforward.

In what follows, we assume that the solution $\boldsymbol{\zeta}^{\varepsilon}$ is "smooth enough" in the sense that $n^{\alpha \beta} \in H^{1}(\omega)$ and $m^{\alpha \beta} \in H^{2}(\omega)$.

(i) We first establish the relations

$$
\partial_{\alpha} \sqrt{a}=\sqrt{a} \Gamma_{\sigma \alpha}^{\sigma} .
$$

Let $\boldsymbol{A}$ denote the matrix of order three with $\mathbf{a}_{1}, \mathbf{a}_{2}, \mathbf{a}_{3}$ as its column vectors, so that $\sqrt{a}=\operatorname{det} \boldsymbol{A}$ (see part (i) of the proof of Theorem 2.10-2). Consequently,

$$
\begin{aligned}
\partial_{\alpha} \sqrt{a} & =\operatorname{det}\left(\partial_{\alpha} \mathbf{a}_{1}, \mathbf{a}_{2}, \mathbf{a}_{3}\right)+\operatorname{det}\left(\mathbf{a}_{1}, \partial_{\alpha} \mathbf{a}_{2}, \mathbf{a}_{3}\right)+\operatorname{det}\left(\mathbf{a}_{1}, \mathbf{a}_{2}, \partial_{\alpha} \mathbf{a}_{3}\right) \\
& =\left(\Gamma_{1 \alpha}^{1}+\Gamma_{2 \alpha}^{2}+\Gamma_{3 \alpha}^{3}\right) \operatorname{det}\left(\mathbf{a}_{1}, \mathbf{a}_{2}, \mathbf{a}_{3}\right)=\sqrt{a} \Gamma_{\sigma \alpha}^{\sigma}
\end{aligned}
$$


since $\partial_{\alpha} \mathbf{a}_{\beta}=\Gamma_{\beta \alpha}^{\sigma} \mathbf{a}_{\sigma}+b_{\alpha \beta} \mathbf{a}_{3}$ (see Section 2.1).

(ii) Using the Green formula in Sobolev spaces (see, e.g., Nečas [73]) and assuming that the functions $n^{\alpha \beta}=n^{\beta \alpha}$ are in $H^{1}(\omega)$, we first transform the first integral appearing in the left-hand side of the variational equations. This gives, for all $\boldsymbol{\eta}=\eta_{i} \mathbf{a}^{i}$ with $\eta_{\alpha} \in H_{0}^{1}(\omega)$ and $\eta_{3} \in L^{2}(\omega)$, hence a fortiori for all $\boldsymbol{\eta}=\eta_{i} \mathbf{a}^{i}$ with $\eta_{\alpha} \in H_{0}^{1}(\omega)$ and $\eta_{3} \in H_{0}^{2}(\omega)$,

$$
\begin{aligned}
& \int_{\omega} a^{\alpha \beta \sigma \tau} \gamma_{\sigma \tau}\left(\boldsymbol{\zeta}^{\varepsilon}\right) \gamma_{\alpha \beta}(\boldsymbol{\eta}) \sqrt{a} d y=\int_{\omega} n^{\alpha \beta} \gamma_{\alpha \beta}(\boldsymbol{\eta}) \sqrt{a} d y \\
& =\int_{\omega} \sqrt{a} n^{\alpha \beta}\left(\frac{1}{2}\left(\partial_{\beta} \eta_{\alpha}+\partial_{\alpha} \eta_{\beta}\right)-\Gamma_{\alpha \beta}^{\sigma} \eta_{\sigma}-b_{\alpha \beta} \eta_{3}\right) d y \\
& =\int_{\omega} \sqrt{a} n^{\alpha \beta} \partial_{\beta} \eta_{\alpha} d y-\int_{\omega} \sqrt{a} n^{\alpha \beta} \Gamma_{\alpha \beta}^{\sigma} \eta_{\sigma} d y-\int_{\omega} \sqrt{a} n^{\alpha \beta} b_{\alpha \beta} \eta_{3} d y \\
& =-\int_{\omega} \partial_{\beta}\left(\sqrt{a} n^{\alpha \beta}\right) \eta_{\alpha} d y-\int_{\omega} \sqrt{a} n^{\alpha \beta} \Gamma_{\alpha \beta}^{\sigma} \eta_{\sigma} d y-\int_{\omega} \sqrt{a} n^{\alpha \beta} b_{\alpha \beta} \eta_{3} d y \\
& =-\int_{\omega} \sqrt{a}\left(\partial_{\beta} n^{\alpha \beta}+\Gamma_{\tau \beta}^{\alpha} n^{\tau \beta}+\Gamma_{\beta \tau}^{\beta} n^{\alpha \tau}\right) \eta_{\alpha} d y-\int_{\omega} \sqrt{a} n^{\alpha \beta} b_{\alpha \beta} \eta_{3} d y \\
& =-\int_{\omega} \sqrt{a}\left\{\left(\left.n^{\alpha \beta}\right|_{\beta}\right) \eta_{\alpha}+b_{\alpha \beta} n^{\alpha \beta} \eta_{3}\right\} d y .
\end{aligned}
$$

(iii) We then likewise transform the second integral appearing in the left-hand side of the variational equations, viz.,

$$
\begin{aligned}
& \frac{1}{3} \int_{\omega} a^{\alpha \beta \sigma \tau} \rho_{\sigma \tau}\left(\boldsymbol{\zeta}^{\varepsilon}\right) \rho_{\alpha \beta}(\boldsymbol{\eta}) \sqrt{a} d y=\int_{\omega} m^{\alpha \beta} \rho_{\alpha \beta}(\boldsymbol{\eta}) \sqrt{a} d y \\
& =\int_{\omega} \sqrt{a} m^{\alpha \beta} \partial_{\alpha \beta} \eta_{3} d y \\
& \quad+\int_{\omega} \sqrt{a} m^{\alpha \beta}\left(2 b_{\alpha}^{\sigma} \partial_{\beta} \eta_{\sigma}-\Gamma_{\alpha \beta}^{\sigma} \partial_{\sigma} \eta_{3}\right) d y \\
& \quad+\int_{\omega} \sqrt{a} m^{\alpha \beta}\left(-2 b_{\beta}^{\tau} \Gamma_{\alpha \tau}^{\sigma} \eta_{\sigma}+\left.b_{\beta}^{\sigma}\right|_{\alpha} \eta_{\sigma}-b_{\alpha}^{\sigma} b_{\sigma \beta} \eta_{3}\right) d y,
\end{aligned}
$$

for all $\boldsymbol{\eta}=\eta_{i} \mathbf{a}^{i}$ with $\eta_{\alpha} \in H_{0}^{1}(\omega)$ and $\eta_{3} \in H_{0}^{2}(\omega)$. Using the symmetry $m^{\alpha \beta}=m^{\beta \alpha}$, the relation $\partial_{\beta} \sqrt{a}=\sqrt{a} \Gamma_{\beta \sigma}^{\sigma}$ (cf. part (i)), and the same Green formula as in part (ii), we obtain

$$
\begin{aligned}
\int_{\omega} m^{\alpha \beta} \rho_{\alpha \beta}(\boldsymbol{\eta}) & \sqrt{a} d y=-\int_{\omega} \sqrt{a}\left(\partial_{\beta} m^{\alpha \beta}+\Gamma_{\beta \sigma}^{\sigma} m^{\alpha \beta}+\Gamma_{\sigma \beta}^{\alpha} m^{\sigma \beta}\right) \partial_{\alpha} \eta_{3} d y \\
& +2 \int_{\omega} \sqrt{a} m^{\alpha \beta} b_{\alpha}^{\sigma} \partial_{\beta} \eta_{\sigma} d y \\
& +\int_{\omega} \sqrt{a} m^{\alpha \beta}\left(-2 b_{\beta}^{\tau} \Gamma_{\alpha \tau}^{\sigma} \eta_{\sigma}+\left.b_{\beta}^{\sigma}\right|_{\alpha} \eta_{\sigma}-b_{\alpha}^{\sigma} b_{\sigma \beta} \eta_{3}\right) d y .
\end{aligned}
$$


The same Green formula further shows that

$$
\begin{aligned}
& -\int_{\omega} \sqrt{a}\left(\partial_{\beta} m^{\alpha \beta}+\Gamma_{\beta \sigma}^{\sigma} m^{\alpha \beta}+\Gamma_{\sigma \beta}^{\alpha} m^{\sigma \beta}\right) \partial_{\alpha} \eta_{3} d y \\
& =-\int_{\omega} \sqrt{a}\left(\left.m^{\alpha \beta}\right|_{\beta}\right) \partial_{\alpha} \eta_{3} d y=\int_{\omega} \partial_{\alpha}\left(\left.\sqrt{a} m^{\alpha \beta}\right|_{\beta}\right) \eta_{3} d y \\
& =\int_{\omega} \sqrt{a}\left(\left.m^{\alpha \beta}\right|_{\alpha \beta}\right) \eta_{3} d y \\
& 2 \int_{\omega} \sqrt{a} m^{\alpha \beta} b_{\alpha}^{\sigma} \partial_{\beta} \eta_{\sigma} d y=-2 \int_{\omega} \sqrt{a}\left\{\partial_{\beta}\left(b_{\alpha}^{\sigma} m^{\alpha \beta}\right)+\Gamma_{\beta \tau}^{\tau} b_{\alpha}^{\sigma} m^{\alpha \beta}\right\} \eta_{\sigma} d y .
\end{aligned}
$$

Consequently,

$$
\begin{aligned}
\int_{\omega} m^{\alpha \beta} \rho_{\alpha \beta}(\boldsymbol{\eta}) \sqrt{a} d y= & \int_{\omega} \sqrt{a}\left\{-\left.2\left(b_{\sigma}^{\alpha} m^{\sigma \beta}\right)\right|_{\beta}+\left(\left.b_{\beta}^{\alpha}\right|_{\sigma}\right) m^{\sigma \beta}\right\} \eta_{\alpha} d y \\
& +\int_{\omega} \sqrt{a}\left\{\left.m^{\alpha \beta}\right|_{\alpha \beta}-b_{\alpha}^{\sigma} b_{\sigma \beta} m^{\alpha \beta}\right\} \eta_{3} d y .
\end{aligned}
$$

Using in this relation the easily verified formula

$$
\left.\left(b_{\sigma}^{\alpha} m^{\sigma \beta}\right)\right|_{\beta}=\left(\left.b_{\beta}^{\alpha}\right|_{\sigma}\right) m^{\sigma \beta}+b_{\sigma}^{\alpha}\left(\left.m^{\sigma \beta}\right|_{\beta}\right)
$$

and the symmetry relations $\left.b_{\beta}^{\alpha}\right|_{\sigma}=\left.b_{\sigma}^{\alpha}\right|_{\beta}$ (Theorem 2.8-2), we finally obtain

$$
\begin{aligned}
\int_{\omega} m^{\alpha \beta} \rho_{\alpha \beta}(\boldsymbol{\eta}) \sqrt{a} d y= & -\int_{\omega} \sqrt{a}\left\{\left.\left(b_{\sigma}^{\alpha} m^{\sigma \beta}\right)\right|_{\beta}+b_{\sigma}^{\alpha}\left(\left.m^{\sigma \beta}\right|_{\beta}\right)\right\} \eta_{\alpha} d y \\
& -\int_{\omega} \sqrt{a}\left\{b_{\alpha}^{\sigma} b_{\sigma \beta} m^{\alpha \beta}-\left.m^{\alpha \beta}\right|_{\alpha \beta}\right\} \eta_{3} d y .
\end{aligned}
$$

(iv) By parts (ii) and (iii), the variational equations

$$
\int_{\omega}\left\{a^{\alpha \beta \sigma \tau} \gamma_{\sigma \tau}\left(\boldsymbol{\zeta}^{\varepsilon}\right) \gamma_{\alpha \beta}(\boldsymbol{\eta})+\frac{1}{3} a^{\alpha \beta \sigma \tau} \rho_{\sigma \tau}\left(\boldsymbol{\zeta}^{\varepsilon}\right) \rho_{\alpha \beta}(\boldsymbol{\eta})-p^{i, \varepsilon} \eta_{i}\right\} \sqrt{a} d y=0
$$

imply that

$$
\begin{gathered}
\int_{\omega} \sqrt{a}\left\{\left.\left(n^{\alpha \beta}+b_{\sigma}^{\alpha} m^{\sigma \beta}\right)\right|_{\beta}+b_{\sigma}^{\alpha}\left(\left.m^{\sigma \beta}\right|_{\beta}\right)+p^{\alpha, \varepsilon}\right\} \eta_{\alpha} d y \\
+\int_{\omega} \sqrt{a}\left\{b_{\alpha \beta} n^{\alpha \beta}+b_{\alpha}^{\sigma} b_{\sigma \beta} m^{\alpha \beta}-\left.m^{\alpha \beta}\right|_{\alpha \beta}+p^{3, \varepsilon}\right\} \eta_{3} d y=0
\end{gathered}
$$

for all $\boldsymbol{\eta}=\eta_{i} \mathbf{a}^{i}$ with $\eta_{\alpha} \in H_{0}^{1}(\omega)$ and $\eta_{3} \in H_{0}^{2}(\omega)$. The announced partial differential equations are thus satisfied in $\omega$.

The functions

$$
n^{\alpha \beta}=\varepsilon a^{\alpha \beta \sigma \tau} \gamma_{\sigma \tau}\left(\zeta^{\varepsilon}\right)
$$

are the contravariant components of the linearized stress resultant tensor field inside the shell, and the functions

$$
m^{\alpha \beta}=\frac{\varepsilon^{3}}{3} a^{\alpha \beta \sigma \tau} \rho_{\sigma \tau}\left(\boldsymbol{\zeta}^{\varepsilon}\right)
$$

are the contravariant components of the linearized stress couple, or linearized bending moment, tensor field inside the shell. 
The functions

$$
\begin{aligned}
\left.t^{\alpha \beta}\right|_{\beta} & =\partial_{\beta} t^{\alpha \beta}+\Gamma_{\beta \sigma}^{\alpha} t^{\beta \sigma}+\Gamma_{\beta \sigma}^{\beta} t^{\alpha \sigma}, \\
\left.t^{\alpha \beta}\right|_{\alpha \beta} & =\partial_{\alpha}\left(\left.t^{\alpha \beta}\right|_{\beta}\right)+\Gamma_{\alpha \sigma}^{\sigma}\left(\left.t^{\alpha \beta}\right|_{\beta}\right),
\end{aligned}
$$

which have naturally appeared in the course of the proof of Theorem 2.10-3, constitute examples of first-order, and second order, covariant derivatives of a tensor field defined on a surface, here by means of its contravariant components $t^{\alpha \beta}: \bar{\omega} \rightarrow \mathbb{R}$.

Finally, we state a regularity result that provides an instance where the weak solution, viz., the solution of the variational equations, is also a classical solution, viz., a solution of the associated boundary value problem. The proof of this result, which is due to Alexandrescu [5], is long and delicate and for this reason is only briefly sketched here.

Theorem 2.10-4. Let $\omega$ be a domain in $\mathbb{R}^{2}$ with boundary $\gamma$ and let $\boldsymbol{\theta}: \bar{\omega} \rightarrow \mathbb{R}^{3}$ be an injective immersion. Assume that, for some integer $m \geq 0$ and some real number $q>1, \gamma$ is of class $\mathcal{C}^{m+4}, \boldsymbol{\theta} \in \mathcal{C}^{m+4}\left(\bar{\omega} ; \mathbb{R}^{3}\right), p^{\alpha, \varepsilon} \in W^{m+1, q}(\omega)$, and $p^{3, \varepsilon} \in W^{m, q}(\omega)$. Finally, assume that $\gamma_{0}=\gamma$. Then the weak solution $\boldsymbol{\zeta}^{\varepsilon}=\zeta_{i}^{\varepsilon} \mathbf{a}^{i}$ found in Theorem 2.10-2 satisfies

$$
\zeta_{\alpha}^{\varepsilon} \in W^{m+3, q}(\omega) \text { and } \zeta_{3}^{\varepsilon} \in W^{m+4, q}(\omega) .
$$

Sketch of proof. To begin with, assume that the boundary $\gamma$ is of class $\mathcal{C}^{4}$ and the mapping $\boldsymbol{\theta}$ belongs to the space $\mathcal{C}^{4}\left(\bar{\omega} ; \mathbb{R}^{3}\right)$.

One first verifies that the linear system of partial differential equations found in Theorem 2.10-3 (which is of the third order with respect to the unknowns $\zeta_{\alpha}^{\varepsilon}$ and of the fourth order with respect to the unknown $\zeta_{3}^{\varepsilon}$ ) is uniformly elliptic and satisfies the supplementing condition on $L$ and the complementing boundary conditions, in the sense of Agmon, Douglis \& Nirenberg [3].

One then verifies that the same system is also strongly elliptic in the sense of Nečas [73], p. 185. A regularity result of Nečas [73], Lemma 3.2, p. 260 then shows that the weak solution $\boldsymbol{\zeta}^{\varepsilon}=\zeta_{i}^{\varepsilon} \mathbf{a}^{i}$ found in Theorem 2.10-2, with components $\zeta_{\alpha}^{\varepsilon} \in H_{0}^{1}(\omega)$ and $\zeta_{3}^{\varepsilon} \in H_{0}^{2}(\omega)$ since $\gamma_{0}=\gamma$ by assumption, satisfies

$$
\zeta_{\alpha}^{\varepsilon} \in H^{3}(\omega) \text { and } \zeta_{3}^{\varepsilon} \in H^{4}(\omega)
$$

if $p^{\alpha, \varepsilon} \in H^{1}(\omega)$ and $p^{3, \varepsilon} \in L^{2}(\omega)$.

A result of Geymonat [51], Theorem 3.5 about the index of the associated linear operator then implies that

$$
\zeta_{\alpha}^{\varepsilon} \in W^{3, q}(\omega) \text { and } \zeta_{3}^{\varepsilon} \in W^{4, q}(\omega)
$$

if $p^{\alpha, \varepsilon} \in W^{1, q}(\omega)$ and $p^{3, \varepsilon} \in L^{q}(\omega)$ for some $q>1$.

Assume finally that, for some integer $m \geq 1$ and some real number $q>1, \gamma$ is of class $\mathcal{C}^{m+4}$ and $\boldsymbol{\theta} \in \mathcal{C}^{m+4}\left(\bar{\omega} ; \mathbb{R}^{3}\right)$. Then a regularity result of Agmon, Douglis \& Nirenberg [3] implies that

$$
\zeta_{\alpha}^{\varepsilon} \in W^{m+3, q}(\omega) \text { and } \zeta_{3}^{\varepsilon} \in W^{m+4, q}(\omega) .
$$

if $p^{\alpha, \varepsilon} \in W^{m+1, q}(\omega)$ and $p^{3, \varepsilon} \in W^{m, q}(\omega)$.

Acknowledgments. With the kind permission of Springer-Verlag, some portions of these notes are extracted and adapted from the book by the first author "An introduction to Differential Geometry with Applications to Elasticity", 
Springer, Dordrecht, 2005, the writing of which was substantially supported by two grants from the Research Grants Council of Hong Kong Special Administrative Region, China [Project No. 9040869, CityU 100803 and Project No. 9040966, CityU 100604].

\section{REFERENCES}

[1] Acerbi, E.; Buttazzo, G.; Percivale, D.: A variational definition of the strain energy for an elastic string, J. Elasticity 25 (1991), 137-148.

[2] Adams, R.A.: Sobolev Spaces, Academic Press, New York, 1975.

[3] Agmon, S.; Douglis, A.; Nirenberg, L. : Estimates near the boundary for solutions of elliptic partial differential equations satisfying general boundary conditions. II, Comm. Pure Appl. Math. 17 (1964), 35-92.

[4] Akian, J.L. : A simple proof of the ellipticity of Koiter's model, Analysis and Applications 1 (2003), 1-16.

[5] Alexandrescu, O. : Théorème d'existence pour le modèle bidimensionnel de coque non linéaire de W.T. Koiter, C.R. Acad. Sci. Paris, Sér. I, 319 (1994), 899-902.

[6] Amrouche, C.; Girault, V. : Decomposition of vector spaces and application to the Stokes problem in arbitrary dimension, Czech. Math. J. 44 (1994), 109-140.

[7] Anicic, S.; Le Dret, H.; Raoult, A. : The infinitesimal rigid displacement lemma in Lipschitz coordinates and application to shells with minimal regularity, Math. Methods Appl. Sci. 27 (2005), 1283-1299.

[8] Attouch, H.: Variational Convergence for Functions and Operators, Pitman, Boston, 1984.

[9] Ball, J.: Convexity conditions and existence theorems in nonlinear elasticity, Arch. Rational Mech. Anal. 63 (1977), 337-403.

[10] Bamberger, Y. : Mécanique de l'Ingénieur, Volume II, Hermann, Paris, 1981.

[11] Bernadou, M.; Ciarlet, P.G. : Sur l'ellipticité du modèle linéaire de coques de W.T. Koiter, in Computing Methods in Applied Sciences and Engineering (R. Glowinski \& J.L. Lions, Editors), pp. 89-136, Lecture Notes in Economics and Mathematical Systems, 134 (1976), Springer-Verlag, Heidelberg.

[12] Bernadou, M.; Ciarlet, P.G.; Miara, B. : Existence theorems for twodimensional linear shell theories, J. Elasticity 34 (1994), 111-138.

[13] Blouza, A.; Le Dret, H. : Existence and uniqueness for the linear Koiter model for shells with little regularity, Quart. Appl. Math. 57 (1999), 317-337.

[14] Bolley, P.; Camus, J. : Régularité pour une classe de problèmes aux limites elliptiques dégénérés variationnels, C.R. Acad. Sci. Paris, Sér. A, 282 (1976), 45-47.

[15] Borchers, W.; Sohr, H. : On the equations $\operatorname{rot} v=g$ and $\operatorname{div} u=f$ with zero boundary conditions, Hokkaido Math. J. 19 (1990), 67-87.

[16] Busse S.: Asymptotic analysis of linearly elastic shells with variable thickness, Revue Roumaine Math. Pures Appl. 43 (1998), 553-590.

[17] Caillerie, D.; Sanchez-Palencia, E. : Elastic thin shells: asymptotic theory in the anisotropic and heterogeneous cases, Math. Models Methods Appl. Sci. 5 (1995), 473-496.

[18] Ciarlet, P.G. : Mathematical Elasticity, Volume I: Three-Dimensional Elasticity, North-Holland, Amsterdam, 1988. 
[19] Ciarlet, P.G. : Mathematical Elasticity, Volume II: Theory of Plates, NorthHolland, Amsterdam, 1997.

[20] Ciarlet, P.G. : Mathematical Elasticity, Volume III: Theory of Shells, NorthHolland, Amsterdam, 2000.

[21] Ciarlet, P.G. : Un modèle bi-dimensionnel non linéaire de coque analogue à celui de W.T. Koiter, C.R. Acad. Sci. Paris, Sér. I, 331 (2000), 405-410.

[22] Ciarlet, P.G. : An Introduction to Differential Geometry with Applications to Geometry, Springer, Dordrecht, 2005 (also appeared as Vol. 78-79 of J. Elasticity, 2005).

[23] Ciarlet, P.G. : An introduction to differential geometry in $\mathbb{R}^{3}$, pp. $x x x-x x x$ in this Volume.

[24] Ciarlet, P.G.; Coutand, D.: An existence theorem for nonlinearly elastic "flexural" shells, J. Elasticity 50 (1998), 261-277.

[25] Ciarlet, P.G.; Destuynder, P. : A justification of the two-dimensional plate model, J. Mcanique 18 (1979), 315-344.

[26] Ciarlet, P.G.; Geymonat, G : Sur les lois de comportement en élasticité nonlinéaire compressible, C.R. Acad. Sci. Paris, Ser. II, 295 (1982), 423-426.

[27] Ciarlet, P.G.; Lods, V. : On the ellipticity of linear membrane shell equations, J. Math. Pures Appl. 75 (1996), 107-124.

[28] Ciarlet, P.G.; Lods, V. : Asymptotic analysis of linearly elastic shells. I. Justification of membrane shell equations, Arch. Rational Mech. Anal. 136 (1996), 119-161.

[29] Ciarlet, P.G.; Lods, V. : Asymptotic analysis of linearly elastic shells. III. Justification of Koiter's shell equations, Arch. Rational Mech. Anal. 136 (1996), 191-200.

[30] Ciarlet, P.G.; Lods, V. : Asymptotic analysis of linearly elastic shells: "Generalized membrane shells", J. Elasticity 43 (1996), 147-188.

[31] Ciarlet, P.G.; Lods, V.; Miara, B. : Asymptotic analysis of linearly elastic shells. II. Justification of flexural shell equations, Arch. Rational Mech. Anal. 136 (1996), 163-190.

[32] Ciarlet, P.G.; Mardare, S. : On Korn's inequalities in curvilinear coordinates, Math. Models Methods Appl. Sci. 11 (2001), 1379-1391.

[33] Ciarlet, P.G.; Miara, B. : On the ellipticity of linear shell models, Z. angew. Math. Phys. 43 (1992), 243-253.

[34] Ciarlet, P.G.; Roquefort, A. : Justification of a two-dimensional nonlinear shell model of Koiter's type, Chinese Ann. Math. 22B (2001), 129-244.

[35] Ciarlet, P.G.; Sanchez-Palencia, E. : An existence and uniqueness theorem for the two-dimensional linear membrane shell equations, J. Math. Pures Appl. 75 (1996), 51-67.

[36] Coutand, D.: Existence of minimizing solutions around "extended states" for a nonlinearly elastic clamped plane membrane. Chinese Ann. Math. B20 (1999), 279-296.

[37] Coutand, D.: Existence of a solution for a nonlinearly elastic plane membrane subject to plane forces. J. Elasticity 53 (1998/99), 147-159.

[38] Dacorogna, B. : Direct Methods in the Calculus of Variations, Springer, Berlin, 1989.

[39] Dal Maso, G.: An Introduction to Г-Convergence, Birkhäuser, Boston, 1993. 
[40] De Giorgi, E.: Sulla convergenza di alcune successioni di integrali del tipo dell' area, Rend. Mat. Roma 8 (1975), 227-294.

[41] De Giorgi, E.: T-convergenza e G-convergenza, Boll. Un. Mat. Ital. 5 (1977), 213-220.

[42] De Giorgi, E. and Dal Maso, G.: $\Gamma$-Convergence and Calculus of Variations, Lecture Notes in Mathematics, Vol. 979, Springer-Verlag, Berlin, 1983.

[43] De Giorgi, E. and Franzoni, T: Su un tipo di convergenza variazionale, Atti Accad. Naz. Lincei Rend. Cl. Sci. Mat. 58 (1975), 842-850.

[44] Destuynder, P.: Sur une Justification des Modèles de Plaques et de Coques par les Méthodes Asymptotiques, Doctoral Dissertation, Université Pierre et Marie Curie, Paris, 1980.

[45] Destuynder, P. : A classification of thin shell theories, Acta Applicandae Mathematicae 4 (1985), 15-63.

[46] Duvaut, G.; Lions, J.L. : Les Inéquations en Mécanique et en Physique, Dunod, Paris, 1972 (English translation: Inequalities in Mechanics and Physics, Springer-Verlag, Berlin, 1976).

[47] Evans L.C.; Gariepy R.F.: Measure Theory and Fine Properties of Functions, CRC Press, 1992.

[48] Friesecke, G.; James, R.D.; Müller, S. : A theorem on geometric rigidity and the derivation of nonlinear plate theory from three-dimensional elasticity. Comm. Pure Appl. Math. 55 (2002), 1461-1506.

[49] Friesecke, G.; James, R.D.; Mora, M.G.; Müller, S. : Derivation of nonlinear bending theory for shells from three dimensional nonlinear elasticity by Gammaconvergence, C.R. Acad. Sci. Paris, Sér. I, 336 (2003), 697-702.

[50] Genevey, K.: Remarks on nonlinear membrane shell problems. Math. Mech. Solids 2, (1997), 215-237.

[51] Geymonat, G. : Sui problemi ai limiti per i sistemi lineari ellittici, Ann. Mat. Pura Appl. 69 (1965), 207-284.

[52] Geymonat, G.; Suquet, P. : Functional spaces for Norton-Hoff materials, Math. Methods Appl. Sci. 8 (1986), 206-222.

[53] Goldenveizer, A.L. : Derivation of an approximate theory of shells by means of asymptotic integration of the equations of the theory of elasticity, Prikl. Mat. Mech. 27 (1963), 593-608.

[54] Grisvard P.: Elliptic Problems in Nonsmooth Domains, Pitman, Boston, 1985.

[55] Gurtin M.E.; Martins L.C.: Cauchy's theorem in classical physics, Arch. Rational Mech. Anal. 60 (1976), 305-328.

[56] John, F. : Estimates for the derivatives of the stresses in a thin shell and interior shell equations, Comm. Pure Appl. Math. 18 (1965), 235-267.

[57] John, F. : Refined interior equations for thin elastic shells, Comm. Pure Appl. Math. 18 (1971), 235-267.

[58] Klingenberg, W. : Eine Vorlesung über Differentialgeometrie, Springer-Verlag, Berlin, 1973 (English translation: A Course in Differential Geometry, SpringerVerlag, Berlin, 1978).

[59] Koiter, W.T. : On the nonlinear theory of thin elastic shells, Proc. Kon. Ned. Akad. Wetensch. B69 (1966), 1-54.

[60] Koiter, W.T. : On the foundations of the linear theory of thin elastic shells, Proc. Kon. Ned. Akad. Wetensch. B73 (1970), 169-195. 
[61] Le Dret, H. : Well-posedness for Koiter and Naghdi shells with a $G_{1^{-}}$ midsurface, Analysis and Applications 2 (2004), 365-388.

[62] Le Dret, H.; Raoult, A.: The nonlinear membrane model as variational limit of nonlinear three-dimensional elasticity, J. Math. Pures Appl. 74 (1995), 549-578.

[63] Le Dret, H.; Raoult, A.: The quasiconvex envelope of the Saint VenantKirchhoff stored energy function, Proc. Royal Soc. Edinburgh 125A (1995), 1179-1192.

[64] Le Dret, H.; Raoult, A. : The membrane shell model in nonlinear elasticity: A variational asymptotic derivation, J. Nonlinear Sci. 6 (1996), 59-84.

[65] Lods, V.; Miara, B. : Nonlinearly elastic shell models. II. The flexural model, Arch. Rational Mech. Anal. 142 (1998), 355-374.

[66] Magenes, E.; Stampacchia, G. : I problemi al contorno per le equazioni differenziali di tipo ellittico, Ann. Scuola Norm. Sup. Pisa 12 (1958), 247-358.

[67] Mardare, S. : Inequality of Korn's type on compact surfaces without boundary, Chinese Annals Math. 24B (2003), 191-204

[68] Marsden,J.E.; Hughes, T.J.R.: Mathematical Foundations of Elasticity, Prentice-Hall, Englewood Cliffs, 1983.

[69] Miara, B. : Nonlinearly elastic shell models. I. The membrane model, Arch. Rational Mech. Anal. 142 (1998), 331-353.

[70] Miara, B.; Sanchez-Palencia, E.: Asymptotic analysis of linearly elastic shells, Asymptotic Anal. 12 (1996), 41-54..

[71] Morrey, Jr., C.B. : Quasi-convexity and the lower semicontinuity of multiple integrals, Pacific J. Math. 2 (1952), 25-53.

[72] Morrey, Jr., C.B.: Multiple Integrals in the Calculus of Variations, SpringerVerlag, Berlin, 1966.

[73] Nečas, J. : Les Méthodes Directes en Théorie des Equations Elliptiques, Masson, Paris, 1967.

[74] Rivlin R.S.; Ericksen J.L.: Stress-deformation relations for isotropic materials, Arch. rational Mech. Anal. 4 (1955), 323-425.

[75] Sanchez-Palencia, E. : Statique et dynamique des coques minces. I. Cas de flexion pure non inhibée, C.R. Acad. Sci. Paris, Sér. I, 309 (1989), 411-417.

[76] Sanchez-Palencia, E. : Statique et dynamique des coques minces. II. Cas de flexion pure inhibée - Approximation membranaire, C.R. Acad. Sci. Paris, Sér. I, 309 (1989), 531-537.

[77] Sanchez-Palencia, E. : Passages à la limite de l'élasticité tri-dimensionnelle à la théorie asymptotique des coques minces, C.R. Acad. Sci. Paris, Sér. II, 311 (1990), 909-916.

[78] Sanchez-Palencia, E. : Asymptotic and spectral properties of a class of singular-stiff problems, J. Math. Pures Appl. 71 (1992), 379-406.

[79] Sanchez-Hubert, J.; Sanchez-Palencia, E. : Coques Elastiques Minces: Proprits Asymptotiques, Masson, Paris, 1997.

[80] Schwartz, L. : Théorie des Distributions, Hermann, Paris, 1966.

[81] Schwartz, L. : Analyse II: Calcul Différentiel et Equations Différentielles, Hermann, Paris, 1992.

[82] Stein, E. : Singular Integrals and Differentiability Properties of Functions, Princeton University Press, 1970.

[83] Stoker, J.J. : Differential Geometry, John Wiley, New York, 1969. 
[84] Tartar, L.: Topics in Nonlinear Analysis, Publications Mathématiques d'Orsay No. 78.13, Université de Paris-Sud, Orsay, 1978.

[85] Taylor M.E.: Partial Differential Equations I: Basic Theory, Springer, Berlin, 1996.

[86] Valent, T. : Boundary Value Problems of Finite Elasticity, Springer Tracts in Natural Philosophy, Vol. 31, Springer-Verlag, Berlin. 1988.

[87] Yosida K.: Functional Analysis, Springer, Berlin, 1978.

Philippe G. Ciarlet, Department of Mathematics, City University of Hong Kong; 83 Tat Chee Avenue, Kowloon, Hong Kong (mapgc@cityu.edu.hk)

Cristinel Mardare, Université Pierre et Marie Curie-Paris6, UMR 7598 Laboratoire JaCQUes-Louis Lions; PARIS, F-75005 France (mardare@ann.jussieu.fr) 